\title{
T-bet and ROR $\alpha$ control lymph node formation \\ by regulating embryonic innate lymphoid cell differentiation
}

\section{DISSERTATION}

zur Erlangung des akademischen Grades

Doctor rerum naturalium

(Dr. rer. nat.)

eingereicht an der

Lebenswissenschaftlichen Fakultät der Humboldt-Universität zu Berlin

von

Christina Stehle, M.Sc.

Präsidentin der Humboldt Universität zu Berlin

Prof. Dr.-Ing. Dr. Sabine Kunst

Dekan der Lebenswissenschaftlichen Fakultät

Der Humboldt-Universität zu Berlin

Prof. Dr. Dr. Christian Ulrichs

Gutachter*innen:

1. Prof. Chiara Romagnani

2. Prof. Andreas Radbruch

3. Prof. Arturo Zychlinsky

Tag der mündlichen Prüfung: 15.09.2021 


\section{Zusammenfassung}

Angeborene lymphoide Zellen (ILCs, engl. für Innate Lymphoid Cells) bilden eine kürzlich beschriebene Familie von Effektorzellen des angeborenen Immunsystems, die in verschiedenste immunologische Prozesse involviert sind. ILCs fehlen somatisch rekombinierte Antigenrezeptoren und können aufgrund ihrer Master-Transkriptionsfaktoren und Effektorfunktionen in drei Hauptgruppen eingeteilt werden. In der Embryonalentwicklung spielt ein spezifisches Mitglied der Typ 3 ILCs, sogenannte LTi (engl. für Lymphoid Tissue inducer) Zellen, eine zentrale Rolle in der Entwicklung von Lymphknoten. Wie die gesamte ILC3 Familie, sind auch LTi Zellen abhängig von dem Master-Transkriptionsfaktor ROR $\gamma$ t, was sich in ROR $\gamma$ t-defizienten Mäuse nicht nur durch die Abwesenheit aller ILC3, sondern makroskopisch auch durch fehlende Lymphknoten äußert. ILC3 persistieren bis ins Erwachsenenalter und befinden sich hauptsächlich im Darmgewebe und den assoziierten mesenterialen Lymphknoten, wo sie die Homöostase der Barrierefunktionen, Immunüberwachung, sowie die Typ-3-Gewebeimmunität unterstützen.

Während postnatale Ko-expression der Transkriptionsfaktoren T-bet und ROR $\gamma$ t in spezifischen ILC3-Subpopulationen und deren Bedeutung für Differenzierung, Phänotyp und Funktionen fest etabliert sind, ist der Einfluss von T-bet in fötalen ILC3 und auf die Generation von Lymphknoten noch unbekannt.

Um diese Mechanismen genau zu untersuchen, wurden fötale ILCs mittels EinzelzellRNA-Sequenzierung detailliert charakterisiert, wodurch eine unerwartete Heterogenität innerhalb der ILC3 mit T-bet-exprimierenden Zellen aufgedeckt wurde. Außerdem wurden PLZF + ILC-Vorläufer (ILCP, engl. für Innate Lymphoid Cell Precursor) im sich entwickelnden Darm nachgewiesen, was darauf hindeutet, dass der embryonale Darm eine aktive Differenzierungsnische für ILCs während der frühen Entwicklung darstellt. Weiterhin, bestätigen verschiedene Mausmodelle eine Schlüsselrolle für T-bet in der Regulation der ILC-Differenzierung und der Entstehung von Lymphknoten. Im Detail konnte gezeigt werden, dass die zusätzliche genetische Ablation von T-bet in ROR $\gamma$ tdefizienten Mäusen Differenzierungsentscheidungen in fötalen ILCP zentral beeinflusst. Die Abwesenheit von T-bet in ILCP ermöglichte die Akkumulation von ILCP mit LTi-Aktivität, wodurch die Organogenese von Lymphknoten, unabhängig von ROR $\gamma$ t wiederhergestellt wurde. PLZF+ ILCP von ROR $\gamma \mathrm{t} / \mathrm{T}$-bet-Doppeldefizienten Mäusen bestanden bis ins Erwachsenenalter, wo diese Zellen die Darmbarrierefunktionen durch Produktion von IL-22 wiederherstellten. Darüber hinaus erwies sich ROR $\alpha$ als entscheidend für die Entwicklung von PLZF+ ILCP und die damit verbundene Bildung von Lymphknoten. Insgesamt enthüllen diese Ergebnisse eine neue Rolle für T-bet und ROR $\alpha$ in der embryonalen ILC-Differenzierung und der Lymphknoten-Organogenese, und decken die antagonistische Funktion von ROR $\gamma$ t innerhalb der differenzierenden ILCP auf, T-bet entgegenzuwirken. 


\section{Summary}

Innate lymphoid cells (ILCs) represent a recently described family of innate effector cells lacking rearranged antigen receptors that are implicated in various immunological processes. ILCs are classified into three main groups based on their lineage-specifying transcription factors (TF) and effector functions. During embryonic development, the formation of lymphoid organs critically relies on a specific member of group 3 innate lymphoid cells (ILC3), expressing the master transcription factor retinoic acid related orphan receptor (ROR) $\gamma$ t and exhibiting lymphoid tissue inducer (LTi) functions. Accordingly, RORyt-deficient mice lack ILC3 and do not generate lymph nodes (LN). ILC3 persist into adulthood and mainly reside within the intestinal tissue and associated mesenteric LN, supporting barrier functions in terms of homeostasis and immunosurveillance as well as type 3 tissue immunity.

While it is established that T-box TF T-bet is co-expressed with ROR $\gamma$ t in a subset of ILC3 emerging postnatally and influencing their differentiation, phenotype and functions, the effect of T-bet on fetal ILC3 biology and its impact on LN generation remains completely unknown.

In order to study the role of T-bet in fetal ILC3 differentiation and functions as well as in LN formation, single-cell RNA sequencing and flow cytometry were applied to characterize fetal ILC subsets revealing an unanticipated heterogeneity within embryonic ILC3 and identifying T-bet ${ }^{+}$ ILC3 subsets within the fetal intestine and mesenteric LN anlage for the first time.

Furthermore, promyelocytic leukaemia zinc finger protein (PLZF) ILC progenitors (ILCP) were exposed in the developing mouse intestine suggesting the embryonic gut as an active differentiation niche for ILCs during early development.

Importantly, using multiple mouse models, a key role for T-bet in regulating ILC differentiation and $L N$ formation was discovered. Specifically, additional deficiency of T-bet in ROR $\gamma t$-deficient mice skewed lineage fate decisions in differentiating fetal ILCP and allowed accumulation of ILCP with LTi activity, thereby rescuing $\mathrm{LN}$ organogenesis in a ROR $\gamma$ t-independent fashion. PLZF ${ }^{+}$ILCP of ROR $\gamma \mathrm{t} / \mathrm{T}$-bet double deficient mice persisted into adulthood where these cells restored intestinal barrier functions through reinstalled IL-22 production. Moreover, ROR $\alpha$ was found to be critical for the development of PLZF' ${ }^{+}$ILCP and associated LN formation.

Altogether, these data unveil a novel role for T-bet and ROR $\alpha$ in embryonic ILC differentiation and $L N$ organogenesis and further highlight the importance of ROR $\gamma t$ in counteracting suppressive effects of T-bet within differentiating ILCP. 


\section{Contents}

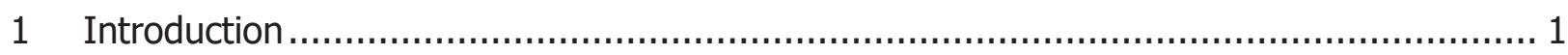

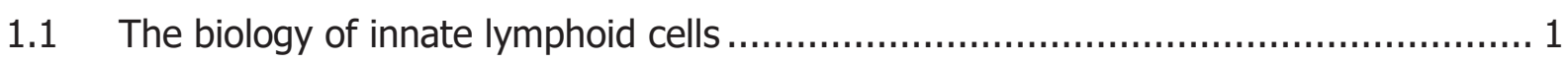

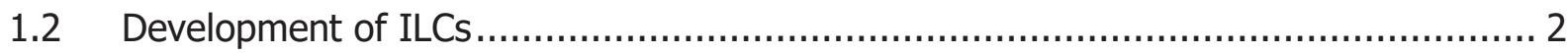

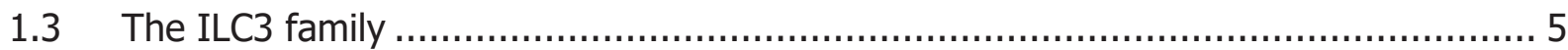

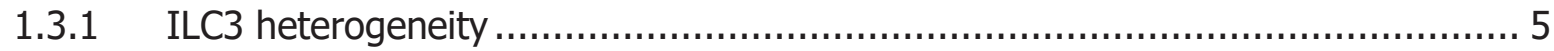

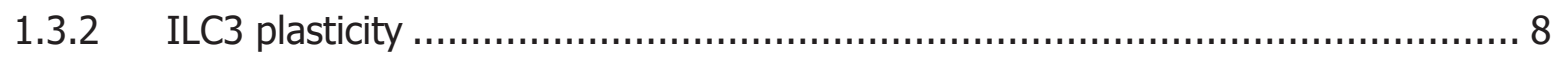

1.3.3 Functions of ILC3 prenatally: lymph node organogenesis ........................10

1.3.4 Functions of ILC3 prenatally: Peyer's patch development ............................11

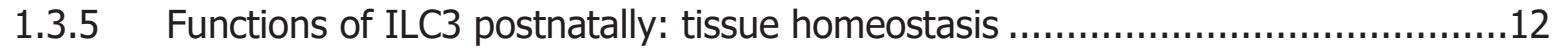

1.3.6 Functions of ILC3 postnatally: protective mucosal immunity ........................14

1.3.7 Dysregulation of ILC3 postnatally: implications in disease ..........................15

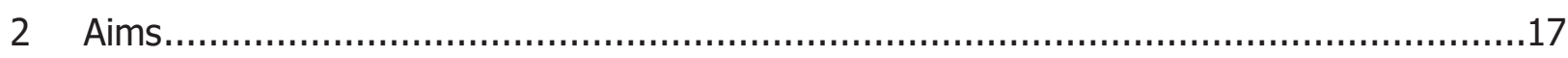

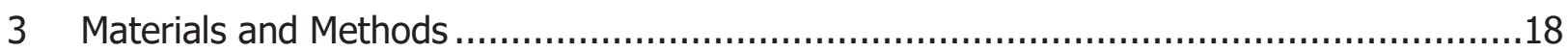

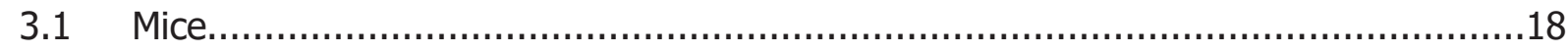

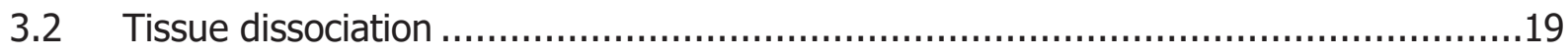

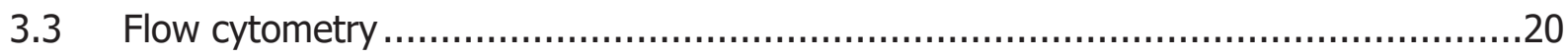

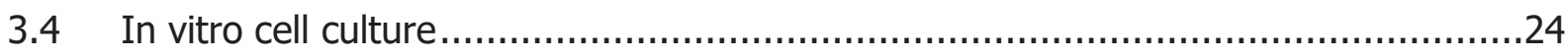

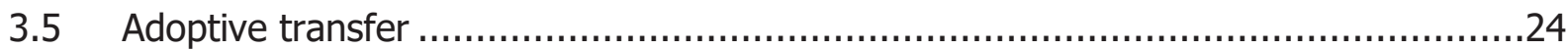

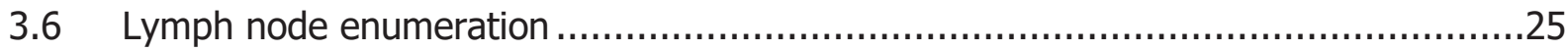

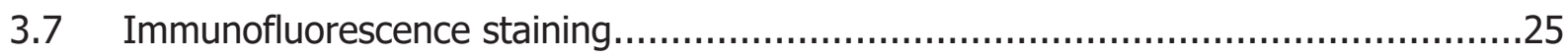

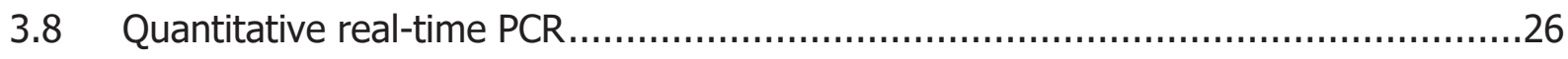

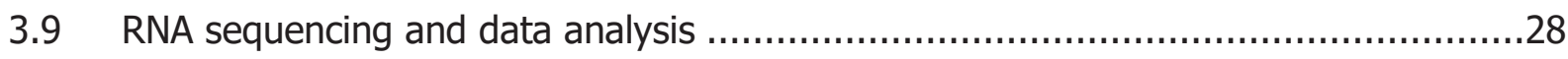

3.10 10x Genomics Chromium single-cell RNA-sequencing ....................................28

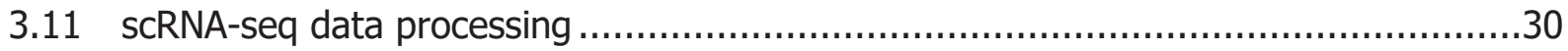

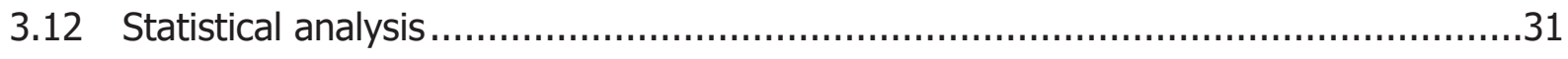

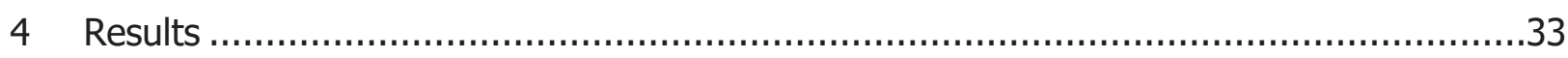

4.1 Identification of fetal ILC subsets in the developing mouse intestine.....................33 
4.2 Single-cell sequencing of fetal intestinal cells reveals a spectrum of mature ILC subsets and progenitors .34

4.3 Transcriptomic profiling on single-cell level discloses heterogeneity within the embryonic ILC3 supercluster.... .39

4.4 A subset of ROR $\gamma \mathrm{t}^{+} \mathrm{T}_{\text {-bet }}{ }^{+} \mathrm{CD} 4^{+}$ILC3 emerges at E14.5 during embryonic development 42

4.5 T-bet deficiency rescues formation of peripheral LN in ROR $\gamma$ t-deficient mice .46

4.6 Block of ILC1 and ILC3 differentiation in $\operatorname{RorC}(g t)^{G F P / G F P} \times \operatorname{Tb} \times 21^{-1-}$ mice favors the accumulation of ILCP .48

4.7 PLZF ${ }^{\text {hi }}$ ILCP from DKO mice are enriched in cells with LTi signatures .....................49

4.8 $\mathrm{PLZF}^{\text {hi }} \mathrm{CXCR6}^{+} \mathrm{GFP}^{+}$cells persist in the $\mathrm{LN}$ and intestine of adult DKO mice.............54

4.9 LN development and accumulation of PLZF ${ }^{\text {hi }}$ ILCP in DKO mice is promoted by ROR $\alpha$

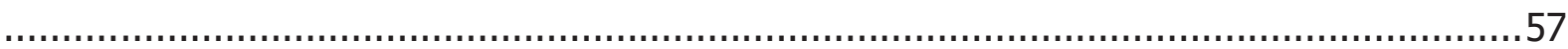

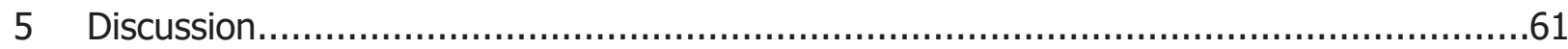

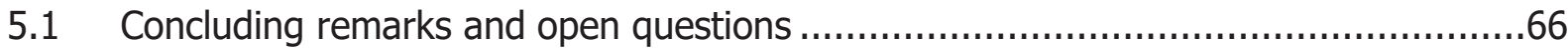

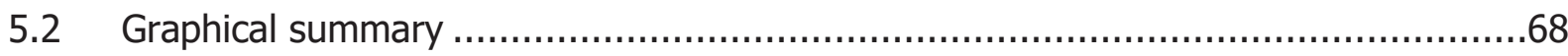

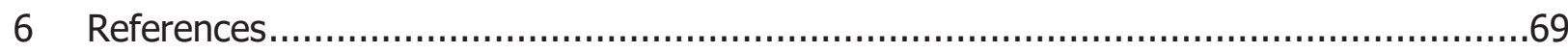

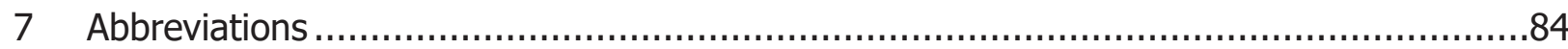

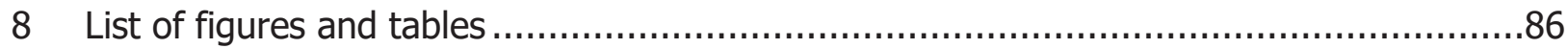

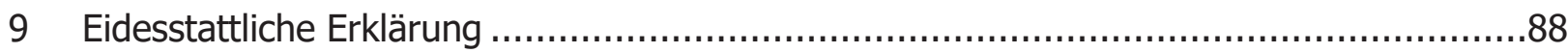




\section{Introduction}

The human immune system is composed of diverse hematopoietic cell types, including lymphocytes from the adaptive as well as from the innate immune system. The family of innate lymphocytes has been expanded recently by the identification of Innate Lymphoid Cells (ILCs), which over the past years emerged as important players not only in the context of infection, cancer and chronic or metabolic diseases, but also in regulation of tissue homeostasis. Intense investigation has contributed to greater understanding of their development, functions and transcriptional regulations in all these processes and further studies will reveal their unique functions as tissue-embedded lymphocytes.

\subsection{The biology of innate lymphoid cells}

Innate lymphoid cells (ILCs) are characterized by the lack of adaptive antigen receptors and hence represent a distinct arm of the innate immune system. In terms of transcriptional regulation and functions, ILCs can be classified into three major groups that mirror T helper (Th) cell subsets and are termed helper-like ILCs accordingly (Figure 1). Group 1 ILCs (ILC1) express IFN $\gamma$ and depend on T-box transcription factor (TF) T-bet, Group 2 ILCs (ILC2) produce IL-4, IL-5, IL-9, IL-13, AREG and express high levels of GATA-3, while Group 3 ILCs (ILC3) are characterized by production of IL-22, IL-17, lymphotoxin and retinoic acid receptor-related orphan receptor (ROR) $\gamma$ t expression (Eberl et al., 2014). The innate counterpart of cytotoxic $\mathrm{CD}^{+} \mathrm{T}$ cells are represented by natural killer (NK) cells, which, like ILC1, produce IFN $\gamma$ and depend on T-bet but furthermore express Eomes and high levels of perforin and granzymes demonstrating their superior cytotoxic capacity to degranulate and to kill aberrant cells (Orange et al., 1995; Spits et al., 2016; Vosshenrich et al., 2006). Based on these effector functions, NK cells and ILC1 contribute to type 1 immunity by reacting to intracellular pathogens, such as viruses, certain bacteria or parasites and are fundamental in anti-tumor immunity. ILC2 on the other hand respond to large extracellular parasites and allergens leading to type 2 immunity (Fallon et al., 2006; Monticelli et al., 2011; Moro et al., 2010; Neill et al., 2010; Price et al., 2010; Saenz et al., 2010), while ILC3 combat extracellular microbes like bacteria and fungi conferring type 3 immunity (Figure 1) (Buonocore et al., 2010; Cupedo et al., 2009; Lee et al., 2011; Satoh-Takayama et al., 2008; Sonnenberg et al., 2011). However, unlike cells of the adaptive immune system, ILCs seed various tissues already during fetal development and remain tissueresident throughout lifetime. Strongly enriched at barrier surfaces such as intestine, lung or skin, ILCs emerged as important players in tissue homeostasis with the ability to rapidly react to environmental changes or tissue perturbations. In this context, in the absence of antigen specificity, ILCs are capable of sensing local chemokine or cytokine milieus, recognize damage- 


\section{Introduction}

associated as well as pathogen-associated molecular patterns (DAMPs and PAMPs) or crosstalk with other (non-) hematopoietic cells to control diverse physiological processes or orchestrate various immune responses (Sonnenberg and Artis, 2015). ILCs act early on and are an important source of cytokines during the initial phases of a multilayered immune response and hence, together with cells of the adaptive immune system, protect organisms not only against infections but also carefully surveil barrier surfaces under homeostatic conditions. Conversely, the dysregulation of ILCs can result in immune pathology and chronic disorders such as or allergies and related asthma.

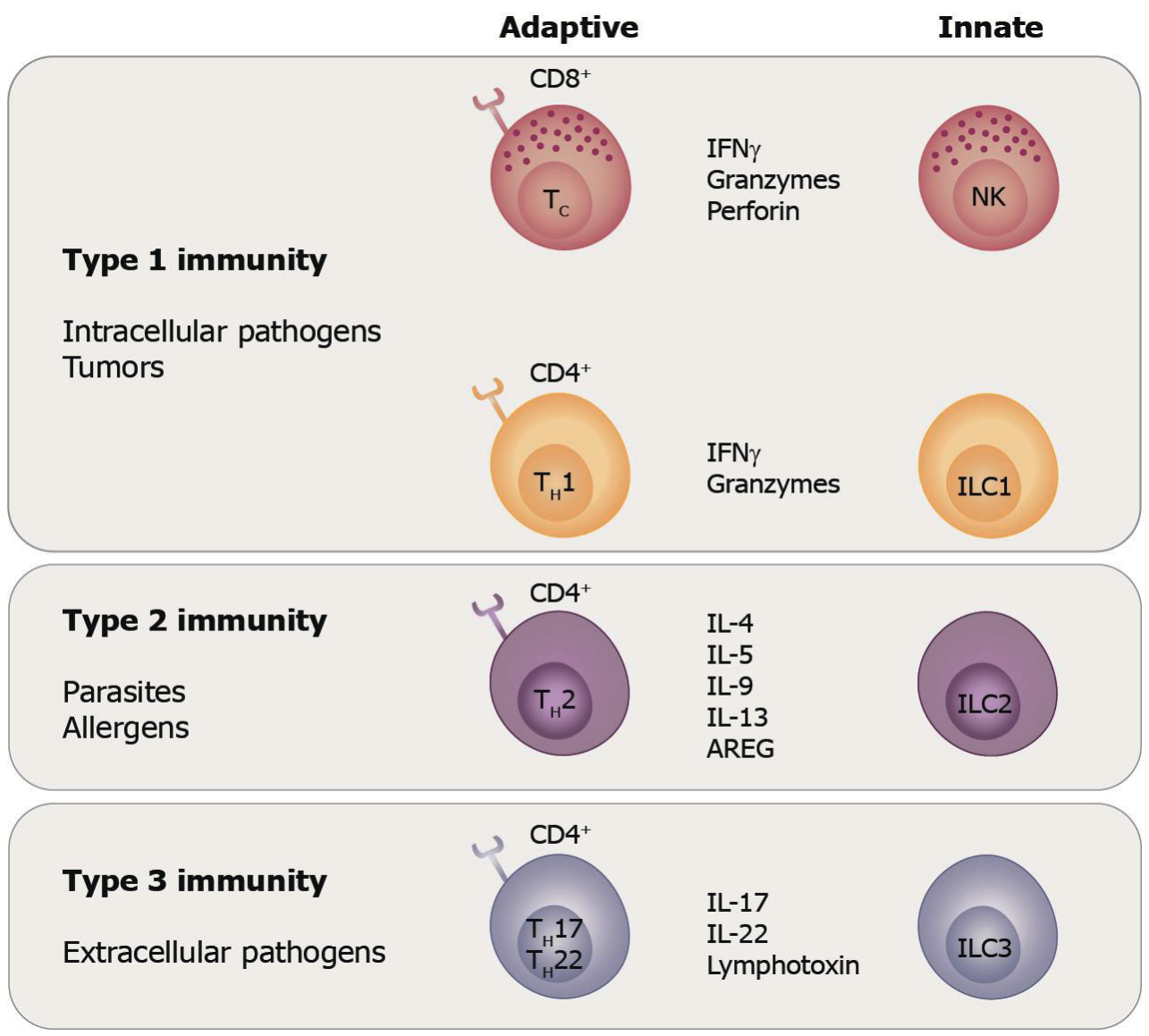

Figure 1. The family of innate lymphoid cells. The diversity of ILCs are mirrored by their $\mathrm{T}$ cell counterparts from the adaptive immune system. While adaptive lymphocytes are also found in the circulation, ILCs mainly reside at barrier surfaces where they orchestrate multiple host-protective effector functions. Both natural killer (NK) cells and ILC1 produce interferon- $\gamma$ (IFN $\gamma$ ) amongst other effector molecules to confer type 1 immunity by reacting to intracellular pathogens, such as viruses or intracellular bacteria, and to tumors. Production of type 2 cytokines, including interleukin (IL)-4, IL-5, IL-9, IL13 or amphiregulin (AREG) promote immunity to helminths and can furthermore be involved in allergic reactions. ILC3 produce IL-17, IL-22 and lymphotoxin to regulate type 3 immunity and control extracellular bacterial infections.

\subsection{Development of ILCs}

The cellular substrate for the formation of all cells from the hematopoietic lineage are multipotent hematopoietic stem cells (HSCs). Hematopoiesis from HSCs is a continuous process marked by the upregulation of several TFs and a progressive loss of cell-fate potentials. Priming towards 
lymphopoiesis and concomitant repression of the myeloid program involves, amongst other TFs, expression of tyrosine kinase FLT3, E2A (also known as TCF3), zinc finger TFs Ikaros and GFI1 (Yoshida et al., 2006). E protein TFs are central components in this step as these promote specification and commitment to adaptive $T$ and $B$ cell fates. The lymphoid-committed resulting cell population is named the common lymphoid progenitor (CLP) and has a restricted potential to generate $T$ and $B$ lymphocytes but also ILCs. Under the influence of IL-2R $\gamma$ C signaling, in the main provided by IL-7, further differentiation towards the ILC lineage is promoted by the inhibitor of DNA-binding 2 (ID2), an ID family member of transcriptional repressors. Specifically, ID2 antagonizes DNA binding activities of $\mathrm{E}$ proteins thereby inhibiting $\mathrm{T}$ and $\mathrm{B}$ cell potential while strongly promoting ILC generation (Boos et al., 2007; Harly et al., 2018; Klose et al., 2014; Yokota et al., 1999). Activation of ID2 is concomitantly promoted by the TF nuclear factor IL-3 NFIL3 (also known as E4BP4) identifying these factors as critical controllers of innate lymphocyte differentiation (Geiger et al., 2014; Seillet et al., 2014a; Xu et al., 2015). Subsequent additional key transcriptional circuitries by a set of genes including TOX, TCF1 and GATA3 dedicates to the innate lymphoid cell progenitor (ILCP) as shown by strong developmental defects in mice genedeficient for one of these TFs (Figure 2) (Aliahmad et al., 2010; Mielke et al., 2013; Seehus et al., 2015; Yagi et al., 2014; Yang et al., 2015). Moreover, several other TFs such as ETS1, ROR $\alpha$ or PLZF have been shown to be expressed by committed ILCPs (Constantinides et al., 2014; Halim et al., 2012; Walker et al., 2019; Wong et al., 2012; Yu et al., 2016; Zook et al., 2016). Succeeding differentiation of ILCPs towards diversification of main ILC groups finally manifests by the expression of lineage-specifying TFs, resulting in emergence of mature ILC subsets with distinct functional properties described above. 


\section{Introduction}

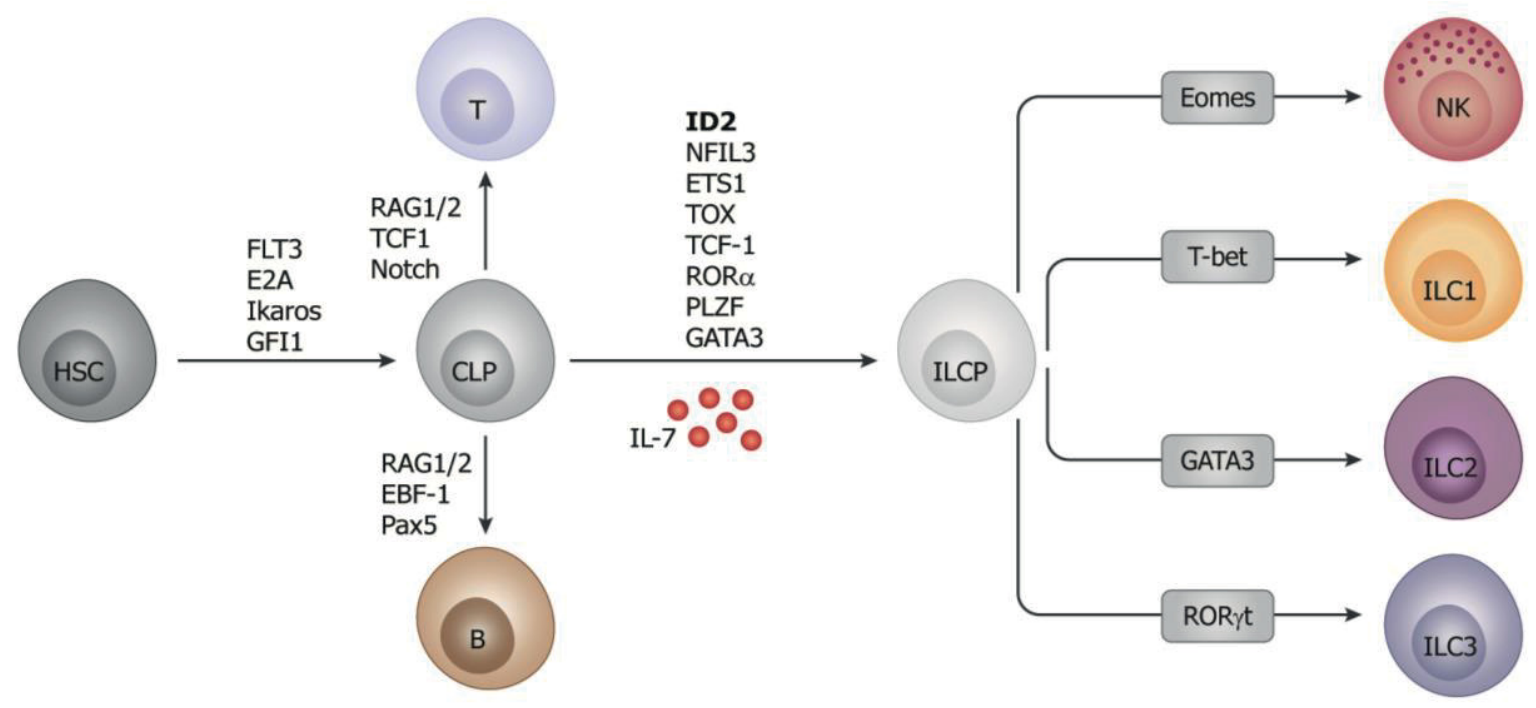

$\begin{array}{cccc}\begin{array}{c}\text { Hematopoietic } \\ \text { progenitor }\end{array} & \begin{array}{c}\text { Lymphoid-restricted } \\ \text { progenitor }\end{array} & \begin{array}{c}\text { Multi-potent ILC } \\ \text { progenitor }\end{array} & \text { Mature ILC } \\ & & & \\ & & \text { Fetal liver } & \text { Intestine } \\ \text { Lung } & \text { Skin } & \text { Fetal LN } & \text { Adipose tissue } \\ \text { Hemogenic endothelium } & \text { Fetal liver } & \text { BM } & \text { Salivary glands } \\ \text { BM } & \text { Fetal LN anlagen } & \text { Tonsil } & \text { Liver } \\ \text { blood } & \text { BM } & \text { Lung } & \text { BM } \\ & & & \end{array}$

Figure 2. Model of ILC development. The developmental program of ILCs is characterized by the sequential expression of transcription factors and successive loss of differentiation potential and is mainly based on studies in mice. The common lymphoid progenitor (CLP) originates from hematopoietic stem cells (HSC) and gives rise to not only B and T cells but also to innate lymphoid cells. Upregulation of ID2 (inhibitor of DNA-binding 2) is crucial for suppression of adaptive cell fates and progression towards the innate lymphoid cell progenitor (ILCP). Subsequent expression of the master transcription factors Eomes (Eomesodermin), T-bet (T-box transcription factors), GATA3 (GATA binding protein 3) and ROR $\gamma$ t (RAR-related orphan receptor $\gamma$ t) define the diverse mature ILC lineages. The different progenitor populations have been found in various locations indicated in the yellow box with early progenitors mainly being detected in the circulation or in niches closely linked to it while the ILCP has been detected in several tissues like tonsil or lung. Mature lineages mainly reside within tissues or at mucosal surfaces such as intestine or lung. FLT3, receptor-type tyrosine-protein kinase FLT3; E2A, E2A immunoglobulin enhancer-binding factors E12/E47; GFI1, Growth Factor Independence 1; RAG1/2, Recombination Activating Gene 1/2; TCF1, T Cell Factor 1; EBF-1, Early B-Cell Factor 1; ID2, Inhibitor of DNA-binding 2; NFIL3, Nuclear Factor, Interleukin 3 Regulated; ETS1, avian erythroblastosis virus E26 homolog-1; TOX, thymocyte selection-associated high mobility group box protein; ROR $\alpha$, RAR-related orphan receptor $\alpha$; PLZF, (promyelocytic leukemia zinc finger; IL-7, interleukin-7.

In embryos, HSCs originate from the yolk sac-derived hemogenic endothelium around embryonic day E10 in mice. As development progresses, CLPs are found in fetal liver (FL) and peripheral LN (Simic et al., 2020) as sites of lymphopoiesis and eventually the BM represents the main niche for hematopoiesis later during ontogeny. Studies in mice and human have identified circulating ILCP (Constantinides et al., 2014; Harly et al., 2018; Klose et al., 2014; Lim et al., 2017; Nagasawa et al., 2019; Renoux et al., 2015; Xu et al., 2019), as well as tissue-resident progenitor pools detected in the fetal mouse intestine (Bando et al., 2015) and adult mouse lung (Zeis et al., 2020) or human tonsil (Montaldo et al., 2014; Renoux et al., 2015; Scoville et al., 2016) suggesting that ILC differentiation may occur at sites other than FL or BM (Figure 2). 


\subsection{The ILC3 family}

Among all lymphocytes, type 3 innate lymphoid cells are the first lymphocytes to appear during embryonic development to organize the formation of lymphoid structures. These cells furthermore confer the first line of defence in newborns until lymphocytes of the adaptive immune system are functionally generated. Later in life, ILC3 are found to be highly enriched in the mucosa of the intestine where they play an essential role in regulating intestinal homeostasis and tissue health but also type 3 immunity. Compared to ILC1 and ILC2, ILC3 represent the most heterogeneous ILC family in adult individuals and can be further divided into three major subpopulations, which are described in the following section.

\subsubsection{ILC3 heterogeneity}

\section{Subsets of ILC3}

In adult mice, ILC3 subsets include natural cytotoxicity receptor (NCR) NKp46 ${ }^{+}$and NKp46- ILC3s (Klose et al., 2013; Sawa et al., 2010) and also lymphoid tissue inducer (LTi) cells. The latter, named after their function to facilitate formation of lymphoid organs during embryonic development, arise in FL and can be characterized by expression of their master regulator ROR $\gamma \mathrm{t}$ and a subset additionally by CD4 on their surface (Table 1) (Cupedo et al., 2004; Eberl et al., 2004; Mebius et al., 2001). LTi cells accumulate in the embryonic LN anlagen and initiate LN organogenesis through surface presentation of lymphotoxin (LT), specifically the heterotrimer LT $\alpha_{1} \beta_{2}$, that signals to specialized stromal cells. Mice deficient for ROR $\gamma$ t fail to develop LTi cells and consequently lack all LN and Peyer's patches (PP) (Eberl et al., 2004; Sun et al., 2000). After birth, a population of $\mathrm{CCR}^{+} \mathrm{CD}^{+/-} \mathrm{ILC3}$, phenotypically resembling LTi cells, is present in spleen, intestine, and LN along with NKp46+ and NKp46- ILC3 subsets (Table 1, Figure 3) (Cupedo et al., 2009). However, LTi cells from the adult stage are unable to facilitate organogenesis of secondary lymphoid organs (Kim et al., 2007; Mariathasan et al., 1995) and rather play a role in regulating the quality and magnitude of adaptive immune responses (Von Burg et al., 2014; Hepworth et al., 2013; Kruglov et al., 2013). The relationship between embryonic LTi cells and those found in adults remains unclarified to date. A model suggests that different waves of ILC3 generation facilitates the generation of functionally different ILC3 subsets, a term called layered ontogeny (McGrath et al., 2015a; Simic et al., 2020). After birth, subsets of non-LTi, described as $\mathrm{NCR}^{+/-}$ILC3, are present and populate gastrointestinal tissues such as small and large intestine as well as $\mathrm{mLN}$ and can furthermore be found in spleen. Importantly, NKp46 ${ }^{+}$ILC3 emerge together with the colonization of the commensal microflora and indispensably express T-bet in addition to RORyt (Sanos et al., 2009; Satoh-Takayama et al., 2008; Sawa et al., 2010).

In humans, LTi cells could be detected within the fetal mesentery and spleen isolated from the first trimester at 8-9 weeks of gestation; however, CCR6 cannot be used as an identifying marker 


\section{Introduction}

for LTi/LTi-like cells since all ROR $\gamma \mathrm{t}^{+}$ILC3 are CCR6+ (Cupedo et al., 2009; Shikhagaie et al., 2017). Instead, NRP-1 has been used herein which has been also shown to be expressed by mouse LTi cells (Robinette et al., 2015; Shikhagaie et al., 2017). Of note, various single-cell RNA sequencing approaches of cells isolated from tissues adult individuals (blood, tonsil, spleen, lung, colon) though have failed to identify a LTi signature as a seperate subcluster from the rest of ILC3 (Bar-Ephraim et al., 2017; Björklund et al., 2016; Mazzurana et al., 2021; Yudanin et al., 2019). Postnatally, human ILC3 subsets are found in tonsils, intestine, skin, LN, spleen, nasal polyps and lung can be distinguished based on CD56 and NKp44 expression, of which the NKp44 subset resembles LTi cells (Cella et al., 2009; Glatzer et al., 2013; Hoorweg et al., 2012). Notably, NKp44+ ILC3 largely co-express NKp46; but, contradictory to mouse NK46 ${ }^{+}$ILC3, human ILC3 do not express T-bet ex vivo.

Taken together, there exists a remarkable diversity among ROR $\gamma \mathrm{t}^{+}$ILC3 postnatally, which can be characterized by a set of surface markers and by co-expression of T-bet in mouse (Table 1).

Table 1. Murine ILC3 markers.

\begin{tabular}{|c|c|c|c|}
\hline & LTi & NCR-ILC3 & $\mathrm{NCR}^{+} \mathrm{ILC3}$ \\
\hline \multicolumn{4}{|l|}{ Markers } \\
\hline CD127 & + & + & + \\
\hline IL-23R & + & + & + \\
\hline IL-1R1 & + & + & + \\
\hline CCR6 & + & - & - \\
\hline CD4 & $+/-$ & - & - \\
\hline CXCR5 & + & - & - \\
\hline NRP-1 & + & - & - \\
\hline NK1.1 & - & - & lo/- \\
\hline NKp46 & - & - & + \\
\hline DNAM1 & - & - & + \\
\hline c-Kit & + & lo & lo \\
\hline CD49a & - & $+/-$ & + \\
\hline CD200R1 & - & + & + \\
\hline \multicolumn{4}{|c|}{ Transcription factors } \\
\hline $\mathrm{ROR} \gamma \mathrm{t}$ & + & + & $+/ l o$ \\
\hline T-bet & - & $+/-$ & + \\
\hline GATA3 & lo & lo & lo \\
\hline \multicolumn{4}{|c|}{ Cytokines } \\
\hline $\mathrm{IFN} \gamma$ & - & $-/ / 0$ & $-/ / 0$ \\
\hline IL-22 & + & + & + \\
\hline IL-17 & + & $+/-$ & - \\
\hline LT & + & $+/-$ & - \\
\hline
\end{tabular}

+ indicates high expression, - indicates no expression, +/- indicates bimodal expression, lo indicates low expression; LT, lymphotoxin Applicable according to published reports (Klose et al., 2013; Robinette et al., 2015; Verrier et al., 2016) 


\section{Antagonizing signals within ILC3 lineages}

In light of the existence of several ILC3 subsets, particularly factors regulating subset classifications were identified. For example, it was proposed that the upregulation of NKp46 and T-bet involves, besides IL-23, Notch signaling and the microbiota (Klose et al., 2013, 2014; Rankin et al., 2013; Sawa et al., 2010; Sciumè et al., 2012). Furthermore, a sustained Notch pathway is required for the maintenance of NKp46+ ILC3 while the cytokine transforming growth factor- $\beta$ (TGF- $\beta$ ) exerts opposing signals inhibiting their generation (Chea et al., 2016a; Viant et al., 2016). With the acquisition of T-bet, NKp46 ${ }^{+}$ILC3 become sensitive to IL-12 and display an "NK-ness" program while transcriptional profiles within NCR-CD4+/- ILC3 subsets are almost identical, as shown using bulk RNA sequencing (Rankin et al., 2016; Robinette et al., 2015). Thus, T-bet seems to regulate ILC3 identity not only phenotypically but also transcriptionally and tunable co-expression of T-bet and RORyt serves as a molecular switch controlling gene expression patterns.

Data obtained in T cells point towards a regulatory role of T-bet in the development of the Th1like Th17 lineage (Lazarevic et al., 2011). Mechanistically, T-bet indirectly inhibits RORyt and consequently represses Th17 lineage. Regulations between ROR $\gamma$ t- and T-bet-driven programs might potentially also exist in ILC3 co-expressing the two TFs, as shown in T-bet-deficient mice, in which NKp46- ILC3 display enhanced expression of ROR $\gamma$ t and are increased in numbers (Schroeder et al., 2020). Interestingly, a role for ROR $\gamma \mathrm{t}$ in counteracting T-bet functions in ILC3 has been proposed, based on the observation that in heterozygous Rorc(gt) ${ }^{G F P / w t}$ mice, the lack of one copy of the gene encoding ROR $\mathrm{t}$, results in a shift in ILC3 subset equilibrium towards NKp46-expressing ILC3 (Walker et al., 2019; Zhong et al., 2016). However, the detailed mechanisms of ROR $\gamma$ t and T-bet regulation within ILC3, in particular prenatally, remain unclarified to date. 
LTi

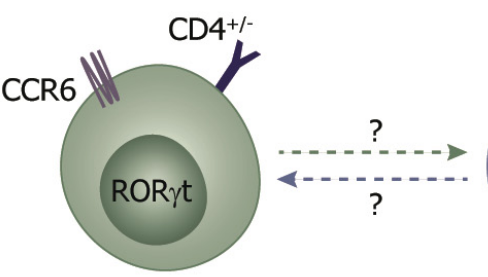

IL-22 Lymphotoxin $\alpha / \beta$
NCR- ILC3

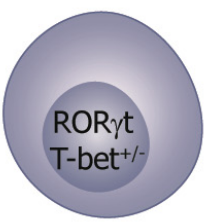

IL-22

IL-17A/F
$\mathrm{NCR}^{+}$ILC3

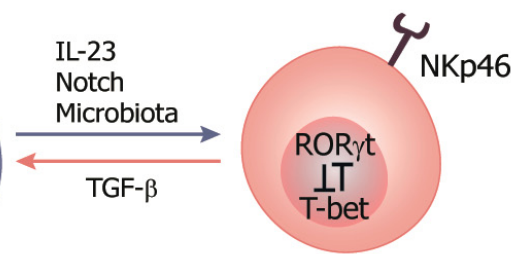

IL-22 IFN $\gamma$

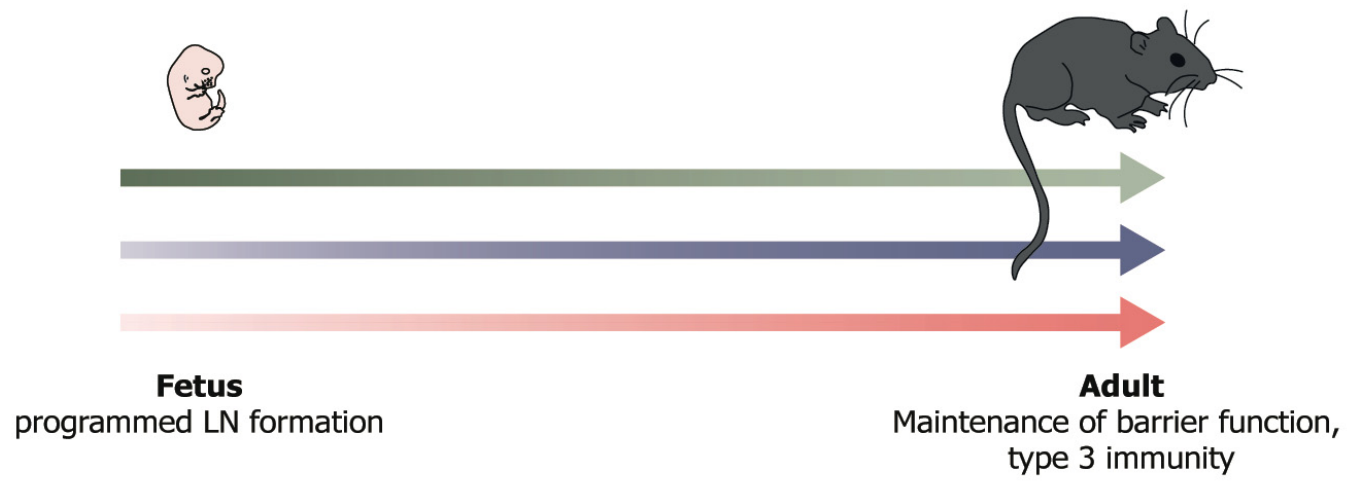

Figure 3. Antagonizing factors regulate ILC3 subsets during ontogeny. Lymphoid tissue inducer (LTi) cells are among the first lymphocytes to develop during ontogeny. These cells unifromly express ROR $\gamma \mathrm{t}$ and are recruited to $\mathrm{LN}$ anlagen where they mature further to produce lymphotoxin enabling formation of lymph nodes. While ROR $\gamma \mathrm{t}^{+} \mathrm{LTi}$ cells represent the majority of ILC3 prenatally, NKp46 ${ }^{+}$ILC3 co-expressing T-bet emerge postnatally with colonization of commensal bacteria, Notch signaling and IL-23. Within $\mathrm{NCR}^{+}$ILC3 ROR $\gamma$ t and T-bet seem to counteract each other's transcriptional programs. Postnatal ILC3 subsets are involved in IL-22 dependent maintenance of barrier function and type 3 immunity. How LTi cells relate to NCR- ILC3 and which signals could drive their interconversion is unclarified to date.

\subsubsection{ILC3 plasticity}

Although generally transcriptionally similar, bulk sequencing of small intestinal NKp46 ${ }^{+}$ILC3 and LTi cells demonstrated distinct transcriptional states within these and single-cell sequencing analyses of small intestinal ILCs furthermore discriminated between five discrete ILC3 transcriptional states (Gury-BenAri et al., 2016; Robinette et al., 2015). This suggests that there might exist dynamic transcriptional and functional states within ILC3, as the broad range of environmental stimuli and/or cellular interactions present in the intestine might require ILC3 to adjust to these conditions and might reflect their need to regulate their identity. As mentioned in section 1.3.1, besides commonly designated NCR ${ }^{+}$ILC3 and LTi cells, a population of NKp46 CCR6-ROR $\mathrm{t}^{+}$ILC3 subsets, termed "double-negative" (DN) ILC3, exists. DN ILC3 have been described to represent a pool of precursors cells that are able to upregulate T-bet and acquire NKp46, a process driven by IL-23, Notch signaling and microbiota (Klose et al., 2013; Rankin et al., 2013; Verrier et al., 2016; Vonarbourg et al., 2010). Reversely, Ncr1 (encoding NKp46) fatemapping studies revealed ILC3 that had switched to a DN ILC3 phenotype upon transforming growth factor- $\beta$ (TGF- $\beta$ ) signals (Verrier et al., 2016; Viant et al., 2016). Interestingly, although 
currently no data on the plasticity within LTi cells have been demonstrated in the literature, some $\mathrm{CCR6}^{+} \mathrm{CD} 4-\mathrm{LTi}$ cells also exhibited a history of NKp46 expression in a study suggestive of plastic changes within the LTi compartment (Verrier et al., 2016).

In addition to the $\mathrm{NCR}^{+/-}$and LTi ILC3 subtypes, an ILC3-ILC1 transitional subset has been described in adult mice and men that resembles ILC1 and is referred to as ex ILC3. ROR $\gamma \mathrm{t}^{-\mathrm{T}}$-bet ${ }^{+}$ ex ILC3 have been shown to differentiate from NKp46 ${ }^{+}$ILC3 in an IL-12 environment downregulating ROR $\gamma \mathrm{t}$ and only expressing T-bet as revealed in fate-mapping studies (Figure 4) (Bernink et al., 2015; Klose et al., 2013, 2014; Vonarbourg et al., 2010). Thus, interconversion of NKp46+ ILC3 to ex-ILC3 producing IFN $\gamma$ is determined by a local cytokine milieu and associated to pathology in mouse models of colitis or disease in Crohn's patients (Bernink et al., 2013; Cella et al., 2019; Verrier et al., 2016; Viant et al., 2016; Vonarbourg et al., 2010). Gene expression analyses suggest that $\mathrm{NKp} 46^{+} \mathrm{ROR} \gamma \mathrm{t}^{\mathrm{lo}} \mathrm{ILC} 3$ might reflect a cell population converting into ex ILC3 (Figure 4) (Robinette et al., 2015). In this context, the TF c-Maf has been reported to balance ILC3/1 subsets by antagonizing T-bet-driven type 1 programs and by enforcing ILC3 identity through support of ROR $\gamma$ t activity and type 3 effector transcriptomes (Parker et al., 2020; Pokrovskii et al., 2019; Tizian et al., 2020). Importantly, human studies have shown that this plastic phenotype is reversible and possibly induced by homeostatic levels of cytokines, highlighting that a blockade of inflammation-polarizing signals could serve as a therapeutic option to resolve inflammation and pathology (Bernink et al., 2013, 2015; Viant et al., 2016). Overall, this phenotypic instability suggests a major role for environmental signals and continual challenges in tuning interconversion of ILC3 subsets along with functional plasticity, and highlights the challenge to categorize the various ILC3 populations based on a very limited number of surface markers.

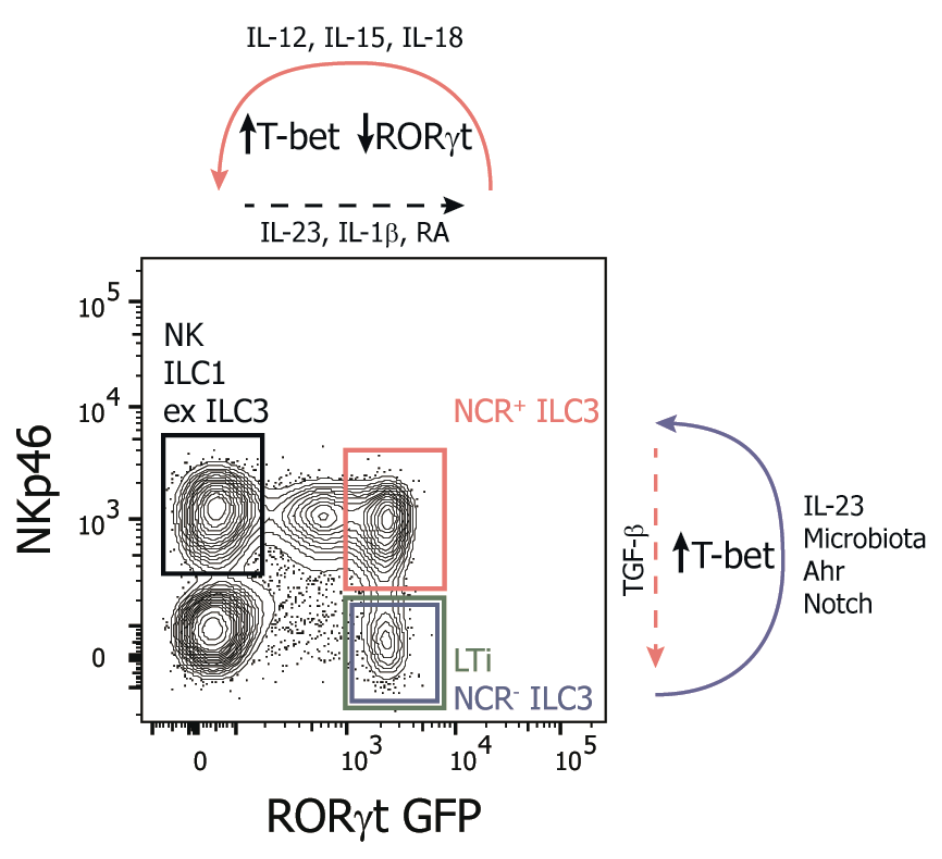

Figure 4. Environmental signals and the interplay of transcription factors ROR $\gamma \mathbf{t}$ and T-bet regulate ILC3 plasticity. Representative flow cytometry plot of ILCs isolated from the small intestine of adult Rorc $(g t)^{G F P / w t}$ reporter mice. Reporter signal ROR $\gamma$ t-GFP defines ILC3 where NKp46subsets consist of lymphoid tissue inducer (LTi) cells and natural cytotoxicity receptor (NCR) $)^{-}$ILC3. Microbiota, Notch and AhR signaling are involved in upregulation of $\mathrm{T}$ bet and consequent differentiation of $\mathrm{NCR}^{+}$ ILC3 expressing NKp46. Through IL-12 signaling this subset is able to turn off ROR $\gamma \mathrm{t}$ expression and convert to ex ILC3. In order to differentiate between ILC1 and ex ILC3 ROR $\gamma$ t-fate map mice should be used. To delineate NK cells from ILC1 transcription factors T-bet and Eomes should be analyzed. RA, retinoic acid; Ahr, aryl hydrocarbon receptor. 


\section{Introduction}

\subsubsection{Functions of ILC3 prenatally: lymph node organogenesis}

Before birth, ROR $\gamma \mathrm{t}^{+}$ILC3 are considered a rather homogeneous population mainly comprising of LTi cells, which can be detected as early as murine embryonic day E12.5 (12.5 days after conception) in FL (Mebius et al., 1997; Veiga-Fernandes et al., 2007). They can be classified based on the expression of CD4 into $\mathrm{LTi}_{0}$ and $\mathrm{LTi}_{4}$ cells (Sawa et al., 2010; Vonarbourg et al., 2010) and guide a highly programmed series of events that includes the interaction between stromal cells and LTi cells, eventually leading to the generation of lymph nodes in the fetus (Boos et al., 2007; Mebius, 2003). In mice, LTi occurrence and initiation of lymphoid organs occurs between E12.5 until E15.5 at specific locations within the embryo. Specifically, LTi cells egress out from the FL and enter the sites of future lymphoid organ development through expression of integrin $\alpha_{4} \beta_{7}$ on LTi cells that enables rolling and firm adhesion on its ligand MadCam-1 expressed by high endothelial venules (Mebius et al., 1996). LTi cells are further attracted by chemokines such as CXCL13 produced by stromal cells while IL-7 additionally stimulates their accumulation and local maturation. Interestingly, expression of CXCL13 (the chemokine binding to CXCR5) in stromal organizer cells as well as RORyt in LTi cells is induced by retinoic acid (RA) signaling in utero (Van De Pavert et al., 2009, 2014). Thus, LTi cell recruitment and maturation is regulated by the mother's food consumption, which eventually controls the size of secondary lymphoid organs and the efficiency of immune responses in adult offspring (Van De Pavert et al., 2014). At place, LTi cells provide the heterotrimer $L T \alpha_{1} \beta_{2}$ (Mebius et al., 1997) to induce activation of stromal organizer cells to differentiate towards specialized lymphoid tissue organizer (LTo) cells (De Togni et al., 2014). Receptor activator of NF-KB (RANK)-RANKL (Ligand) interactions between LTi and LTo cells further orchestrate lymph node development by stimulating LT $\alpha_{1} \beta_{2}$ expression and LTi cell accumulation (Onder et al., 2017). Although molecular mechanisms of interactions between LTi and LTo cells have been a subject of studies over several years, the detailed sequence of events that generate individual specific LN has remained unclear. In this context, it appears that in different mouse knockout models specific LN are present or absent suggesting that distinct processes operate during particular LN generation (Table 2). For example, the developmental pathways for $\mathrm{mLN}$ and PP differ in their requirements for chemokines/growth factors (Coles et al., 2010) (see next section 1.3.4 below).

Taken together, LTi cells are central in inducing the signals for lymph node development and several adhesion molecules and chemokines are needed for their attraction, retention and activation within the developing lymph node during embryogenesis (Figure 5). 


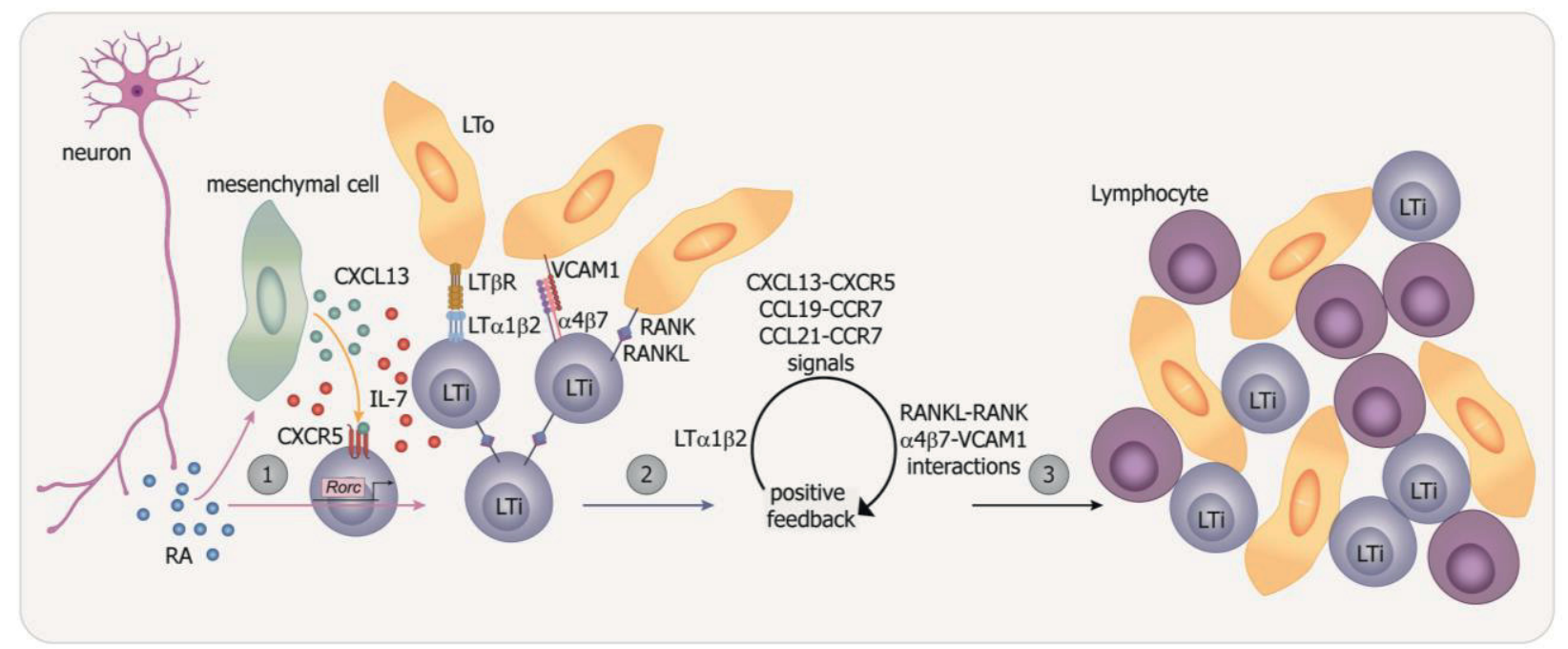

Figure 5. Model for development of secondary lymphoid organs. (1) Induction of organizing centers. Retinoic acid (RA), possibly produced by nerve fibers, induces expression of CXCL13 by mesenchymal cells to attract LTi cells from blood. Local IL-7- and RA-signaling in LTi cells leads to their maturation and expression of the heterotrimer lymphotoxin (LT) $\alpha_{1} \beta_{2}$ on their surface. (2) Clustering of LTi and LTo cells. LT $\beta$ signaling provided to stromal cells leads to their differentiation into lymphoid tissue organizer (LTo) cells. Further interactions via VCAM1$\alpha_{4} \beta_{7}$ and RANK-RANKL facilitates retention and accumulation of LTi cells. (3) Architectural maturation and entry of lymphocytes. Eventually, a large cell cluster forms and blood vessels start to develop into high endothelial venules, which allows cells to enter from the bloodstream.

\subsubsection{Functions of ILC3 prenatally: Peyer's patch development}

PP are organized lymphoid follicles located within the mucosa layer of the small intestine, which harbor not only $\mathrm{T}$ and $\mathrm{B}$ cells but also dendritic cells (DCs), macrophages and neutrophils, thus facilitating crosstalk between these immune cells. Microfold (M) cells are located within PP and actively transport antigen into the follicles to initiate immune responses indicating PP as important locations in immune surveillance. In mice, E15.5 marks the beginning of PP development. First, VCAM-1+LT $\beta R^{+}$stromal cells stochastically locate to the antimesenteric side of the small intestine (Adachi et al., 1997; Patel et al., 2012). Second, between E15.5 and E17.5 CD11 ${ }^{+}$CD127-CD4-ROR $\gamma t^{-}$termed lymphoid tissue initiator (LTin) cells providing RET signaling together with ROR $\gamma t^{+} \mathrm{LTi}$ cells providing $\mathrm{LT} \beta$ signaling to VCAM-1+LT $\beta R^{+}$stromal cells leads to their maturation into LTo cells and to the induction of PP anlagen (Hashi et al., 2001; Patel et al., 2012; Veiga-Fernandes et al., 2007). LTo cells in turn express chemokines, adhesion molecules and cytokines required for recruitment, retention and survival of LTi cells. In this regard, intact LT $\beta, C X C R 5$, as well as IL-7R pathways have been shown to be essential for proper PP development (Table 2). From E17.5 on, circulating lymphocytes are attracted to enter the structures and fill up the niches (Adachi et al., 1997). Interestingly, inhibition of RA signaling only reduces LN dimensions but completely abolishes PP development suggestive of different maternal-derived nutrient requirements in the development of these lymphoid structures (Van De Pavert et al., 2014). 


\section{Introduction}

Overall, LTi cells form the basic architecture during embryonic development and induce PP structures (variable numbers ranging from 5-12 in mice) before entry of mature lymphocytes after birth. Interestingly, our current understanding revealed differing mechanisms in the development of LN versus PP (Table 2).

Table 2. Development of LTi cells, $\mathrm{mLN}$ and PP in mutant mice.

\begin{tabular}{|c|c|c|c|c|}
\hline & LTi & mLN & PP & References \\
\hline$I d 2^{--}$ & - & - & - & (Yokota et al., 1999) \\
\hline $\operatorname{Rorc}(g t)^{G F P / G F P}$ & - & - & - & (Eberl et al., 2004) \\
\hline$I / 7 r^{\circ-}$ & + & + & - & (Cao et al., 1995) \\
\hline$I / 7 /-$ & + & + & + & (von Freeden-Jeffry et al., 1995) \\
\hline$L t \alpha^{-/-}$ & + & - & - & (Banks et al., 1995) \\
\hline$L t \beta^{-1-}$ & + & + & - & (Koni et al., 1997) \\
\hline CxCr5 $5^{-}$ & + & + & $0-2$ & (Ansel et al., 2000) \\
\hline $\mathrm{CxCl} 13^{--}$ & + & - & - & (Förster et al., 1996) \\
\hline Tnfsf1 $11^{--}$ & + & - & + & (Kim et al., 2000) \\
\hline $\mathrm{Kit}^{W} / \mathrm{kit}^{W}$ & + & + & ++ & (Finke et al., 2002) \\
\hline Tnf/- & + & + & reduced & (Pasparakis et al., 1997) \\
\hline
\end{tabular}

\subsubsection{Functions of ILC3 postnatally: tissue homeostasis}

\section{IL-22-mediated barrier surveillance}

After birth, LTi cell frequency and absolute numbers drop and ILC3 heterogeneity increases simultaneous to establishment of symbiotic microbiota (Sawa et al., 2010). In adult individuals, ILC3 mainly locate to intestinal tissues as well as mLN and spleen with an expected half-life of 3-4 weeks in mice (Sawa et al., 2010). There, both human and mouse ILC3 subsets all tonically produce IL-22 under homeostatic conditions including $\mathrm{NCR}^{+/-}$and LTi-like ILC3 to act as major regulators in protection against commensal microbes (Satoh-Takayama et al., 2008; Sawa et al., 2011). In more detail, IL-22 availability in the intestinal tissue is tuned by the microbiota through IL-1 $\alpha$ and IL-23 released from dendritic cells or macrophages, which both stimulate ILC3. IL-22 in turn acts selectively on non-hematopoietic cells like epithelial or stromal cells to induce production of antimicrobial peptides (AMP), such as Reg proteins or $\beta$-defensins, thereby controlling bacterial amounts and maintaining epithelial barrier homeostasis. Loss of ILC3derived IL-22 results in barrier breakdown and translocation of intestinal bacteria to peripheral organs causing systemic inflammation (Sanos et al., 2009; Satoh-Takayama et al., 2008; Sonnenberg et al., 2012). Additionally, IL-22 triggers fucosylation of the intestinal epithelium an important mechanism to maintain host-microbiota symbiosis, as commensal bacteria adapted 
to utilize fucose as a dietary carbohydrate while pathogenic bacteria lack this ability (Goto et al., 2014; JM et al., 2014; Pham et al., 2014). Optimal production of IL-22 by ILC3 has been shown to be modulated by a variety of dietary-derived vitamins, for example vitamin $D$ or also vitamin A-derived RA, the latter similar to LTi regulation in utero (Konya et al., 2018; Van De Pavert et al., 2014; Spencer et al., 2014). Moreover, the TF aryl hydrocarbon receptor (Ahr) can be activated in ILC3 by compounds derived from cruciferous vegetables such as broccoli to regulate IL-22 expression in ILC3 (Kiss et al., 2011; Lee et al., 2011; Qiu et al., 2012, 2013). Overall, ILC3 subsets in the gastrointestinal tract limit the replication and dissemination of commensal bacteria and thus are essential for the maintenance of barrier function and tissue homeostasis in an IL-22dependent manner (Figure 6). Cytokine production can be modulated by multiple factors in response to food intake.

\section{ILC3 regulation of $B$ cell-derived IgA}

An additional layer of intestinal homeostasis is provided by IgA-producing B cells that control commensal microbiota size and composition. On the one hand, ILC3 are positioned within PPs where they produce soluble $\mathrm{LT} \alpha_{3}$ thereby controlling T cell homing to the gut to promote T celldependent IgA production by B cells (Kruglov et al., 2013). On the other hand, clustered LTi cells facilitate development of structures denoted as Cryptopatches (CP) that develop in the first week of life, which will mature into isolated lymphoid follicles (ILFs) as they become populated with B cells producing IgA in response to microbial and dietary signals (Bouskra et al., 2008; Kanamori et al., 1996; Kiss et al., 2011). Formation of ILF from CP requires LT $\beta$ R signaling in stromal cells provided by clustered LTi cells presenting membrane bound $L T \alpha_{1} \beta_{2}$ on their surface (Kang et al., 2002; Lügering et al., 2010; Tsuji et al., 2008). Taken together, ILC3 directly regulate ILF development and indirectly IgA levels in the gastrointestinal tract via membrane bound LT $\alpha_{1} \beta_{2}$ and soluble LT $\alpha_{3}$ (Figure 6).

\section{ILC3 regulation of commensal bacteria-specific $T$ cells}

ILC3-regulate intestinal homeostasis not only by production of IL-22 and LT but also by directly interacting with T cells. In particular, LTi cells localize in close proximity to T cells in order to modulate and orchestrate their immune responses through multiple pathways. For example, LTi cells constitutively express major histocompatibility complex class II (MHCII) endowing them the ability to present antigen to $\mathrm{CD} 4^{+} \mathrm{T}$ cells thereby limiting commensal bacteria-specific $\mathrm{CD} 4^{+} \mathrm{T}$ cell responses (Figure 6). T cell death is on the one hand induced due to absence of costimulatory molecules upon MHCII presentation and secondly by out competition for local IL-2 necessary for T cell proliferation by ILC3 (Von Burg et al., 2014; Hepworth et al., 2013, 2015). Lack of ILC3-intrinsic MHCII in mice results in development of microbiota-dependent T-bet-driven colitis and identify intestinal ILC3 as important players that limit pathological adaptive immune responses to commensal bacteria. 


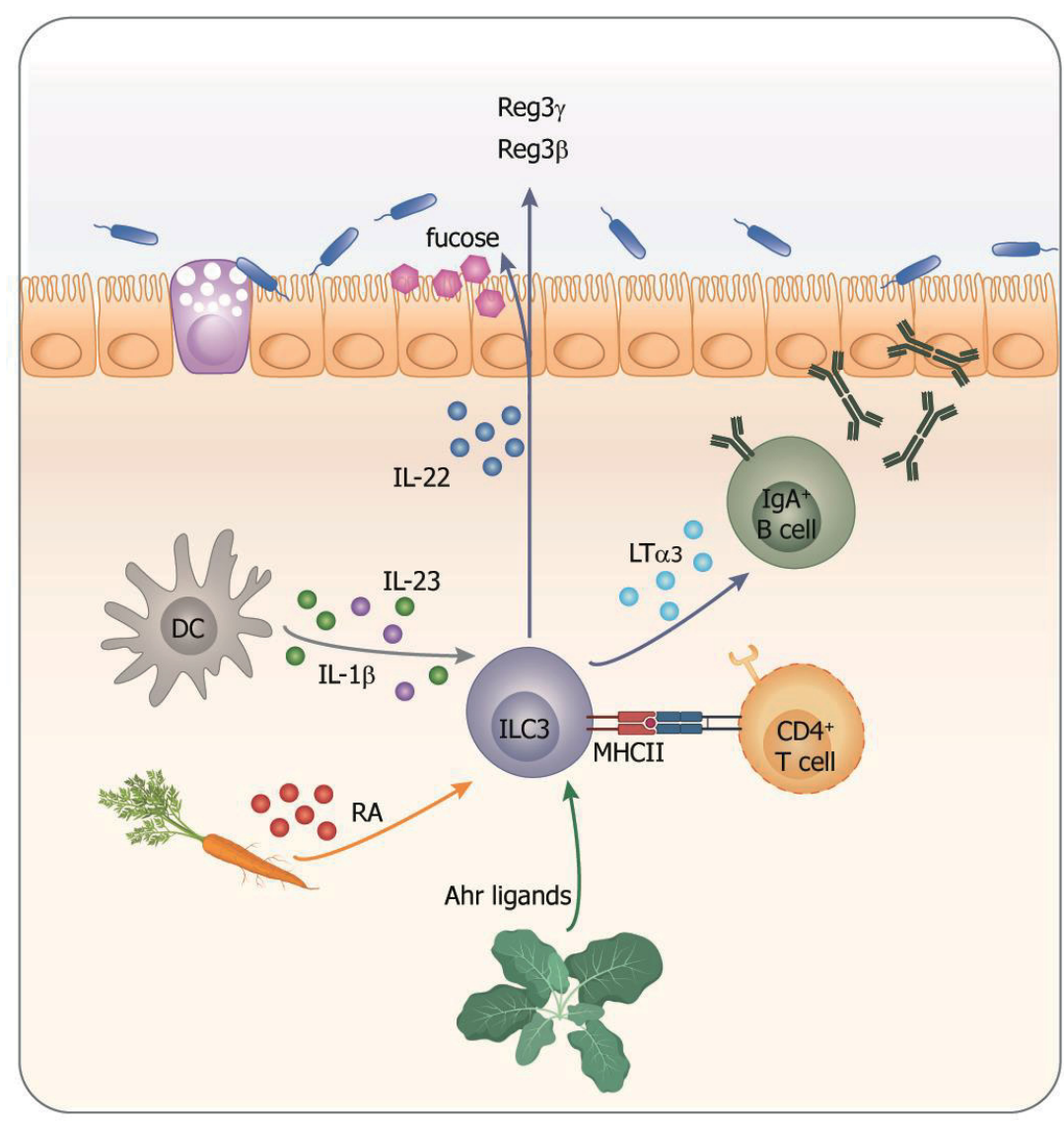

Figure 6. ILC3 in gut homeostasis. Under homeostatic conditions ILC3 are central orchestrators of intestinal tolerance. In particular, ILC3 are a dominant source of interleukin (IL)-22 at steady state. IL-22 acts on epithelial cells to produce antimicrobial peptides such as Reg3 $\gamma$ and Reg $3 \beta$ and furthermore induces fucosylation to maintain segregation of commensal bacteria. In addition, ILC3 regulate IgA production of B cells via soluble $\mathrm{LT}_{3}$ and limit $\mathrm{CD} 4^{+}$commensal T cells in a cell-cell contact dependent manner mediated by major histocompatibility complex (MHC)II.

\subsubsection{Functions of ILC3 postnatally: protective mucosal immunity}

While ILC3 emerged as crucial cells in the maintenance of tissue homeostasis and gut-mucosal tolerance, they can furthermore be involved in inflammation and confer immunity to pathogens. The contribution of ILC3-derived IL-22 in host protective immunity has been assessed in various infection models including Citrobacter rodentium (a model organism for clinically relevant enteropathic Escherichia coli infections) (Satoh-Takayama et al., 2008; Sawa et al., 2011; Sonnenberg et al., 2011), Enterococcus faecalis (Pham et al., 2014) and rotavirus (Hernández et al., 2015). Of note, in the presence of the adaptive immune system, especially T cells, ILC3 are dispensable for the control of $C$. rodentium infection; however, ILC3-derived IL-22 is required to control the early phase of infection before antigen-specific $T$ cell responses are raised and thus represent a first line of defense, especially in newborns (Basu et al., 2012; Cella et al., 2009; Klose et al., 2014; Rankin et al., 2016; Song et al., 2015). These data suggest complementary and redundant functions of ILC3 in multilayered immune responses including innate as well as adaptive lymphocytes. In addition to IL-22, ILC3 are also able to secrete IL-17, a cytokine crucial 
for resistance to fungal oropharyngeal infections such as with Candida albicans (Gladiator et al., 2013; Okada et al., 2015). Remarkably, ILC3-derived IL-17 also showed airway protective responses against Klebsiella pneumoniae (Xiong et al., 2016). Furthermore, T-bet co-expressing ILC3 are concomitantly able to produce IFN $\gamma$, a cytokine essential to control Salmonella typhimurium infection (Goto et al., 2014; Klose et al., 2013).

\subsubsection{Dysregulation of ILC3 postnatally: implications in disease}

The continuous stimulation of ILC3 triggers functional responses that under normal conditions promote barrier integrity and homeostasis; however, dysregulated IL-22/IL-17-producing ILC3 are associated with multiple chronic human inflammatory diseases such as inflammatory bowel disease (IBD) including Crohn's disease (CD) and Ulcerative colitis (UC) (Geremia et al., 2011). Detrimental ILC3 responses have also been associated in several mouse models of colitis, induced by Helicobacter hepaticus, Helicobacter typhlonius, S. typhimurium or anti-CD40, and related to production of IL-17, GM-CSF and IFN $\gamma$ (Buonocore et al., 2010; Klose et al., 2013; Pearson et al., 2016; Powell et al., 2012; Vonarbourg et al., 2010). Despite the beneficial effects of IL-22 in homeostasis, unfavorable effects of this cytokine after tissue damage have been reported. In this context, ILC3-derived IL-22 was implicated in development of colorectal cancer (Grivennikov et al., 2012; Hernandez et al., 2018; Kirchberger et al., 2013). Moreover, harmful ILC3 responses have been observed in the skin of patients with psoriasis in which IL-22 revealed as a key driver of epidermal thickening and identified ILC3 to participate in pathology (Teunissen et al., 2014; Villanova et al., 2014). Additional IL-17 production establishes ILC3 as harmful innate lymphocytes in the skin from mouse models of psoriasis-like disease (Pantelyushin et al., 2012). Further, using a mouse model of obesity-induced asthma, IL-17 producing ILC3 expand in response to IL-1 $\beta$-producing macrophages to drive airway hyper-responsiveness (Kim et al., 2014).

In summary, inappropriate activation of ILC3 may contribute to chronic (intestinal) inflammation (Figure 7) and highlight the importance to understand the circumstances and signals leading to dysregulation and pro- versus anti-inflammatory ILC3 phenotypes in order to prevent or treat human diseases. 


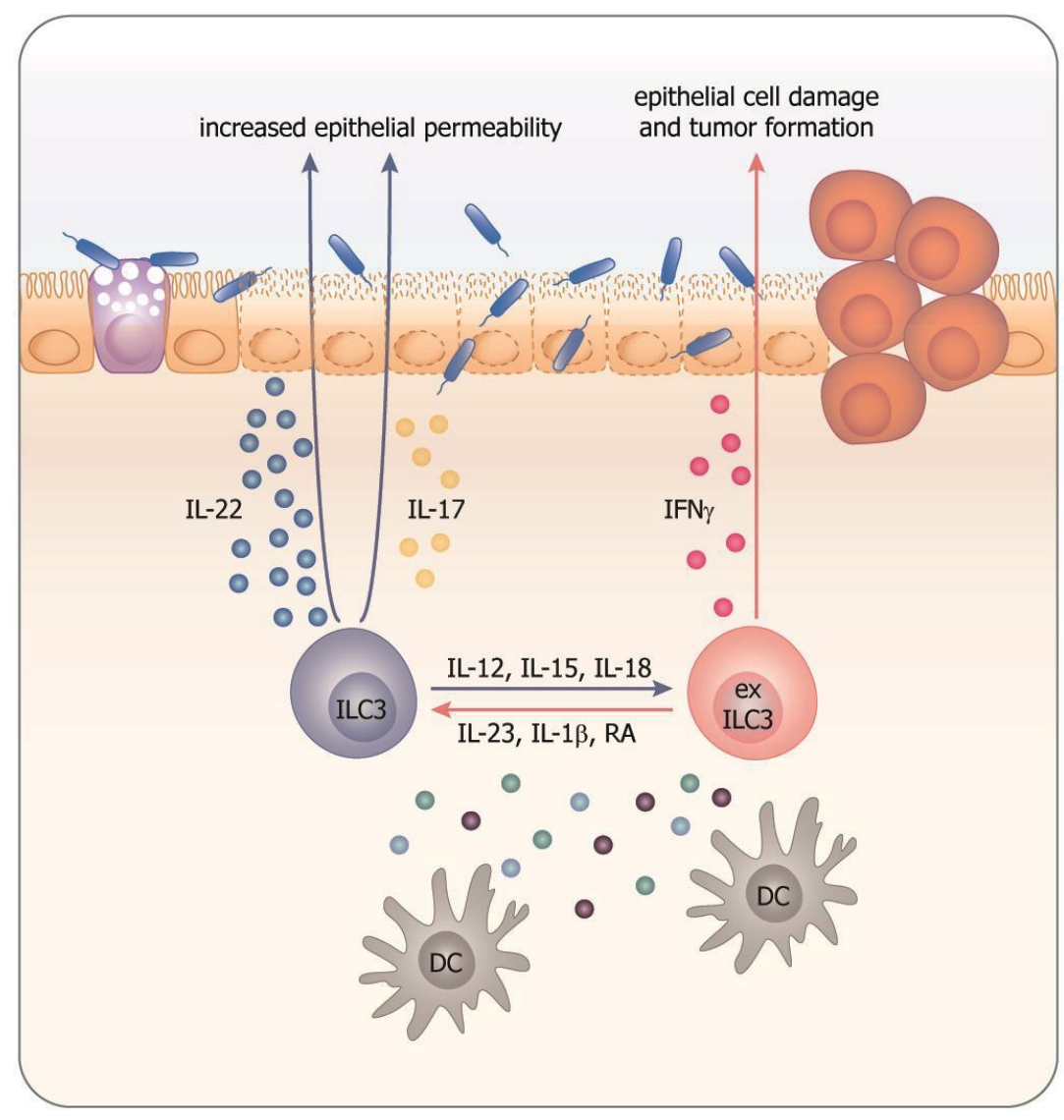

Figure 7. ILC3 in inflammation. Under inflammatory conditions, activated ILC3 may induce pathology through excessive production of IL-22 and IL-17 increasing epithelial permeability resulting in failure to maintain intestinal barrier. Furthermore, dysregulated IL-22 and prolonged chronic inflammation inducing ILC3 conversion to ILC1 can result in epithelial cell damage and potentially progress to colorectal cancer. 


\section{Aims}

Whereas mature ILC3 isolated from adult mice have been extensively studied, their development, heterogeneity and plasticity during embryonic development are poorly understood. To date, LTi cells expressing the master transcription factor ROR $\gamma \mathrm{t}$ are considered a rather homogenous cell population that develops early in fetal life and plays a crucial and non-redundant role in LN formation. However, whether heterogeneous ILC3 subsets displaying differential transcriptomic as well as phenotypic properties establish already before birth remains unclear. While the antagonistic interplay of ROR $\gamma$ t and T-bet in ILC3 has only been elucidated postnatally, the roles of these two TFs during fetal development, especially in LTi cells, have not been investigated to date.

Hence, the overall aim of this thesis was firstly to characterize embryonic ILCs with a particular focus on ILC3, in order to define their transcriptional and phenotypic heterogeneity by applying in-depth analyses such as multicolor flow cytometry as well as single-cell RNA sequencing. Secondly, as T-bet-expressing ILC3 subsets emerged during this approach, the effect of T-bet on embryonic ILC3 function, namely LN formation, was assessed through analysis of multiple mouse models. Thirdly, these steps led to the identification of a complex interplay between ROR $\gamma$ t, T-bet and ROR $\alpha$, acting already early on as crucial regulators of ILCP differentiation during embryogenesis. 


\section{Materials and Methods}

\subsection{Mice}

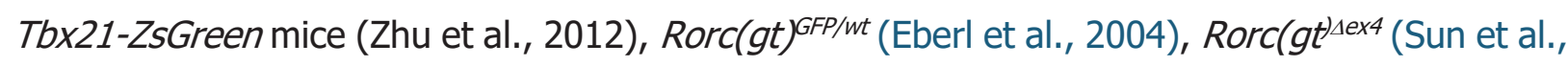
2000), Tbx21\% (Finotto et al., 2002) and Rag2/- (Shinkai et al., 1992) were generated as described. Rorc(gt) ${ }^{c r e}$ (Eberl and Litman, 2004) and Tbx21cre (Haddad et al., 2013) mice were crossed to $R 26 R^{\text {EYFP }}$ and used for $\operatorname{Rorc}(g t)$ - or $T b \times 21$-fate mapping studies. Rorc $(g t)^{\text {cre }}$ was carried only by female breeders to prevent germline YFP expression. To obtain mice with specific deletion of Rorc and/or Tbx21 in I/7r-expressing cells, Rorcf/fl (Choi et al., 2016) and/or Tbx21//ft (purchased from Jackson, stock ID 022741) and/or Roraf/fl (Oliphant et al., 2014) were crossed to $I / 7 r^{\text {cre }}$ (Schlenner et al., 2010) mice in cooperation with David Withers, Birmingham. Rag2 ${ }^{-1}$ $\mathrm{I} / 2 \mathrm{rg}^{\prime-}$ mice were crossed to express the CD45.1 in our facility. Timed pregnancies were accomplished by mating in the evening; evidenced copulation was checked in the morning and females then considered E0.5. All mice were bred under specific pathogen-free conditions in the animal facility of the Federal Institute for Risk Assessment (Berlin, Germany) and the Research Institute for Experimental Medicine (FEM) of the Charité (Berlin, Germany). Animal handling and experiments were conducted according to the German animal protection laws and approved by the responsible governmental authority (Landesamt für Gesundheit und Soziales).

Table 3. Experimental models: mouse strains

\begin{tabular}{|c|c|c|}
\hline Reagent or resource & Source & Identifier \\
\hline Tbx21-ZsGreen & (Zhu et al., 2012) & $\mathrm{N} / \mathrm{A}$ \\
\hline $\operatorname{Rorc}(g t)^{G F P / w t}$ & (Eberl et al., 2004) & JAX \#007572 \\
\hline $\operatorname{Rorc}(g)^{4 \operatorname{sex} 4}$ & (Sun et al., 2000) & JAX \#007571 \\
\hline$T b \times 21^{\%}$ & (Finotto et al., 2002) & JAX \#004648 \\
\hline $\operatorname{Rag} 2^{-1-}$ & (Shinkai et al., 1992) & MGI \#1858556 \\
\hline $\operatorname{Rorc}(g t)^{\text {cre }}$ & (Eberl and Litman, 2004) & JAX \#022791 \\
\hline$T b \times 21^{c r e}$ & (Haddad et al., 2013) & JAX \#024507 \\
\hline$R 26 R^{e Y F P}$ & (Srinivas et al., 2001) & JAX \#006148 \\
\hline$I / 7 r^{c r e}$ & (Schlenner et al., 2010) & MGI \#4441349 \\
\hline
\end{tabular}




\begin{tabular}{|c|c|c|}
\hline $\operatorname{Rorc}^{f l / f I}$ & (Choi et al., 2016) & JAX \#008771 \\
\hline$T b \times 21^{f / f f}$ & $\mathrm{~N} / \mathrm{A}$ & JAX \#022741 \\
\hline Rora $^{f / f f}$ & (Oliphant et al., 2014) & $\mathrm{N} / \mathrm{A}$ \\
\hline $\operatorname{Rag}^{-1-} \mathrm{II} 2 \mathrm{rg}^{/-}$ & $N / A$ & JAX \#002479 \\
\hline
\end{tabular}

\subsection{Tissue dissociation}

Single cell suspensions from intestinal lamina propria of adult animals were obtained as described before (Paclik et al., 2015). In short, intestines were isolated and PPs were removed. Tissue was cleaned, longitudinally opened and chopped into pieces of $2 \mathrm{~cm}$, followed by two rounds à 15 min at $37^{\circ} \mathrm{C}$ of epithelial cell dissociation using Hank's balanced salt solution without calcium and magnesium (HBSS-/-, Gibco) supplemented with 2\% FCS, 10 mM HEPES buffer (Sigma), 1 mM DTT (ThermoFisher) and 5 mM EDTA (Sigma). After washing with HBSS-/-, tissues were digested using a lamina propria dissociation kit (Miltenyi) according to the manufacturer's instructions. Lymphocytes were further enriched on a 40\%/80\% Percoll (GE Healthcare) gradient.

Small intestines from fetal mice were isolated from embryos under a dissecting microscope. Surrounding mesenteric tissue was removed under further magnification and cut open longitudinally. Tissue was incubated for $30 \mathrm{~min}$ at $37^{\circ} \mathrm{C}$ under agitation in HBSS containing calcium and magnesium (HBSS+/+), 2\% FCS, 10 mM HEPES, $0.2 \mathrm{U} / \mathrm{mL}$ Liberase DL (Sigma) and $50 \mu \mathrm{g} / \mathrm{mL}$ DNaseI (Sigma). Leftover tissue was dissociated using a $22 \mathrm{G}$ needle and cell suspension was passed through a $70 \mu \mathrm{m}$ strainer and washed in PBS/BSA. E14.5 and E16.5 time points were pooled samples; E18.5 intestines were treated separately.

Fetal liver was isolated under a dissecting microscope and a single cell suspension was generated by vigorously pipetting using a $1000 \mu \mathrm{L}$ pipette. Cell suspension was filtered through a $70 \mu \mathrm{m}$ strainer, washed in PBS/BSA followed by erythrocyte lysis.

Embryonic lymph node anlagen from the mesenteric region were dissected under a microscope or isolated from adult animals and passed through a $70 \mu \mathrm{m}$ strainer.

Table 4. Tissue isolation reagents.

\begin{tabular}{|l|l|l|}
\hline Reagent or resource & Source & Identifier \\
\hline Miltenyi Si LPL Kit & Miltenyi & Cat. No. \#130-097-410 \\
\hline HBSS-/- & Gibco & Cat. No. \#14065-056 \\
\hline HBSS+/+ & Gibco & Cat. No. \#14065-056 \\
\hline
\end{tabular}




\begin{tabular}{|l|l|l|}
\hline HEPES & Sigma & Cat. No. \#54457 \\
\hline DTT & ThermoFisher & Cat. No. \#R0861 \\
\hline FCS & ThermoFisher & Cat. No. \#16010159 \\
\hline EDTA & Sigma & Cat. No. \#PHR2586 \\
\hline Liberase DL & GE Healthcare & Cat. No. \#17-0891-01 \\
\hline DNaseI & Sigma & Cat. No. \#05466202001 \\
\hline PBS/BSA & Sigma & Cat. No. \#DN25 \\
\hline Gentle MACS & In house & N/A \\
\hline
\end{tabular}

\subsection{Flow cytometry}

Cells were stained using the following antibodies: APC-Vio770 anti-mouse CD19 (REA749), APCFire780 anti-mouse F4/80 (BM8), APC-Fire780 anti-mouse Gr-1 (RB6-8C5), APC-Cy7 anti-mouse FCERI $\alpha$ (MAR-1), BV510 or BUV396 anti-mouse CD45 (30-F11), APC anti-mouse CD45.1 (A20), VioGreen anti-mouse CD45.2 (104-2), PerCP-eF710 anti-mouse $\gamma \delta$ TCR (GL-3), A700 or PerCPCy5.5 anti-mouse $\beta$ TCR (H57-597), PerCP-Vio700 anti-mouse CD3 (REA641), PE-Dazzle594 or BV605 or PE anti-mouse NKp46 (29A1.4), BV650, BV711, BV785 or PE-Cy7 or AlexaFlour647 anti-mouse CD4 (RM4-5), BV650 or BV711 anti-mouse CD8 (53-6.72), BV785 anti-mouse CD127 (A7R34), PE-Vio770 anti-mouse CD122 (REA1015), PE anti-mouse CCR6 (29-2L17), PE-Vio615 anti-mouse CXCR5 (REA215), PerCP-eF710 anti-mouse CXCR6 (DANID2), Alexa700 anti-mouse CD90 (T24), BV421 anti-mouse $\alpha_{4} \beta_{7}$ (DATK32), PE-Cy7 or BV605 anti-mouse KLRG1 (2F1/KLRG1), BUV661 or BV785 anti-mouse NK1.1 (PK136), PE-Vio770 anti-human/rat/mouse ICOS (REA192), BUV563 anti-mouse CD38 (90/CD38), BV711 anti-mouse IgD (11-26c.2a), PerCP-eF710 anti-mouse GL7 (GL7), PE anti-mouse Fas (Jo2), BV510 or BV711 or PE-Cy7 antimouse PD1 (29F.1A12). For staining with PE anti-mouse RANKL (IK22/5) and LT $\alpha_{1} \beta_{2}$-hFab (Lee et al., 2006) and anti-hIgG APC (IS11-12E4.23.20) cells were incubated for $1 \mathrm{~h}$ at $37^{\circ} \mathrm{C}$ in complete RPMI medium containing 10\% FCS. To minimize non-specific binding of antibodies, cells were blocked with anti-mouse CD16/32 (2.4G2) and dead cells were excluded by staining with Fixable Viability Dye (LD) (ThermoFisher) prior to staining.

For intranuclear staining, cells were fixed with transcription factor staining buffer set (BD Bioscience) according to the manufacturer's instructions. For fixation of fate-map YFP signal, 
cells were fixed $20 \mathrm{~min}$ at room temperature in $2 \%$ para-formaldehyde (EMS) and stained in BD Bioscience perm buffer with PE-Vio615 or FITC anti mouse/human GATA3 (REA174), AlexaFluor647 anti-mouse T-bet (4B10), PE or BV650 anti-mouse ROR $\gamma \mathrm{t}$ (Q31-378), eFluor450 Eomes (Dan11mag), AlexaFluor647 anti-mouse/human PLZF (R17-809), APC anti-mouse Bcl-6 (REA373), A700 anti-mouse Ki67 (16A8). Unless otherwise stated all antibodies were purchased at Miltenyi, Themofisher, Biolegend UK or BD Biosciences.

For the assessment of IL-22 and IL17A-producing cells, single-cell suspensions obtained from small intestinal lamina propria were stimulated in 10\% FCS IMDM and $10 \mathrm{ng} / \mathrm{mL}$ of PMA (SigmaAldrich) and $500 \mathrm{ng} / \mathrm{mL}$ ionomycin (Sigma-Aldrich) in the presence of GolgiPlug containing brefeldin $\mathrm{A}$ (BD Biosciences) for $4 \mathrm{~h}$ at $37^{\circ} \mathrm{C}$. Following washing, cells were surface labelled with above listed antibodies and fixed in 1x InsideFix (Inside Stain Kit Miltenyi Biotec) for 20 min at ambient temperature. Intracellular staining using APC anti-mouse IL-22 (IL22JOP) and BV711 anti-mouse IL-17A (TCC11-18H10.1) was performed in InsidePerm (Inside Stain Kit Miltenyi Biotec) for $30 \mathrm{~min}$ at room temperature.

Flow cytometry was performed using a LSR Fortessa and BD FACSymphony flow cytometer (BD Biosciences). Data were further analysed with Flow Jo. All flow cytometry analysis was performed according to the Guidelines for the use of flow cytometry and cell sorting in immunological studies (Cossarizza et al., 2017).

Table 5. Flow cytometry resources.

\begin{tabular}{|l|l|l|}
\hline Reagent or resource & Source & Identifier \\
\hline APC-Vio770 anti-mouse CD19 (REA749) & Miltenyi & Cat. No. \#130-112-038 \\
\hline APC-Fire780 anti-mouse F4/80 (BM8) & BioLegend & Cat. No. \#123116 \\
\hline APC-Fire780 anti-mouse Gr-1 (RB6-8C5) & BioLegend & Cat. No. \#108456 \\
\hline APC-Cy7 anti-mouse FCERI $\alpha$ (MAR-1) & BioLegend & Cat. No. \#134326 \\
\hline BV510/BUV396 anti-mouse CD45 (30-F11) & BioLegend/BD & Cat. No. \#103138 / 564279 \\
\hline APC anti-mouse CD45.1 (A20) & Miltenyi & Cat. No. \#130-102-470 \\
\hline $\begin{array}{l}\text { VioGreen anti-mouse CD45.2 (104-2) } \\
\text { PerCP-eF710 anti-mouse } \gamma \delta \text { TCR (GL-3) }\end{array}$ & Miltenyi & Cat. No. \#130-102-312 \\
\hline $\begin{array}{l}\text { A700/PerCP-Cy5.5 anti-mouse } \beta \text { TCR (H57- } \\
597)\end{array}$ & BioLegend & Cat. No. \#109224 / 109228 \\
\hline PerCP-Vio700 anti-mouse CD3 (REA641) & Miltenyi & Cat. No. \#130-120-826 \\
\hline
\end{tabular}




\begin{tabular}{|c|c|c|}
\hline $\begin{array}{l}\text { BV605/PE/PE-Dazzle594 anti-mouse NKp46 } \\
\text { (29A1.4) }\end{array}$ & BioLegend & $\begin{array}{l}\text { Cat. No. \#137619 / } 137604 \\
\text { / } 137629\end{array}$ \\
\hline $\begin{array}{l}\text { BV650/BV711/PE-Cy7/AlexaFlour647/BV785 } \\
\text { anti-mouse CD4 (RM4-5) }\end{array}$ & BioLegend & $\begin{array}{l}\text { Cat. No. \#100546 / } 100550 \\
\text { / } 100528 \text { / } 100530 \text { / } 100552\end{array}$ \\
\hline BV650/BV711 anti-mouse CD8 (53-6.72) & BioLegend & Cat. No. \#100742 / 100759 \\
\hline BV785 anti-mouse CD127 (A7R34) & BioLegend & Cat. No. \#135037 \\
\hline PE-Vio770 anti-mouse CD122 (REA1015) & Miltenyi & Cat. No. \#130-117-013 \\
\hline PE anti-mouse CCR6 (29-2L17) & BioLegend & Cat. No. \#129804 \\
\hline PE-Vio615 anti-mouse CXCR5 (REA215) & Miltenyi & Cat. No. \#130-107-656 \\
\hline PerCP-eF710 anti-mouse CXCR6 (DANID2) & ThermoFisher & Cat. No. \#46-9186-82 \\
\hline Alexa700 anti-mouse CD90 (T24) & In house & $\mathrm{N} / \mathrm{A}$ \\
\hline BV421 anti-mouse $\alpha_{4} \beta_{7}$ (DATK32) & $\mathrm{BD}$ & Cat. No. \#566294 \\
\hline $\begin{array}{l}\text { PE-Cy7/BV605 anti-mouse KLRG1 } \\
(2 \mathrm{~F} 1 / \text { KLRG1) }\end{array}$ & BioLegend & Cat. No. \#138416 / 138419 \\
\hline BUV661/BV785 anti-mouse NK1.1 (PK136) & BD/BioLegend & Cat. No. \#741477 / 108749 \\
\hline PE anti-mouse RANKL (IK22/5) & BioLegend & Cat. No. \#510006 \\
\hline LT $\alpha_{1} \beta_{2}-\mathrm{hFab}$ & $\begin{array}{l}\text { (Lee et al., } \\
\text { 2006) }\end{array}$ & $\mathrm{N} / \mathrm{A}$ \\
\hline anti-hIgG APC (IS11-12E4.23.20) & Miltenyi & Cat. No. \#130-119-857 \\
\hline PE-Vio770 anti-mouse ICOS (REA192) & Miltenyi & Cat. No. \#130-100-735 \\
\hline BUV563 anti-mouse CD38 (90/CD38) & $\mathrm{BD}$ & Cat. No. \#741271 \\
\hline BV711 anti-mouse IgD (11-26c.2a) & BioLegend & Cat. No. \#405731 \\
\hline PerCP-eF710 anti-mouse GL7 (GL7) & ThermoFisher & Cat. No. \#46-5902-82 \\
\hline PE anti-mouse Fas (Jo2) & $\mathrm{BD}$ & Cat. No. \# 554258 \\
\hline $\begin{array}{l}\text { BV510 or BV711 anti-mouse PD1 } \\
(29 F .1 A 12)\end{array}$ & BioLegend & Cat. No. \#135241 / 135231 \\
\hline
\end{tabular}




\begin{tabular}{|c|c|c|}
\hline PE-Cy7 anti-mouse PD1 (J43) & ThermoFisher & Cat. No. \# 25-9985-82 \\
\hline BV421 anti-mouse ST2 (DIH9) & BioLegend & Nat. No. \#145309 \\
\hline $\begin{array}{l}\text { PE-Vio615/FITC anti mouse/human GATA3 } \\
\text { (REA174) }\end{array}$ & Miltenyi & $\begin{array}{l}\text { Cat. No. \#130-109-115 / } \\
130-120-147\end{array}$ \\
\hline AlexaFluor647 anti-mouse T-bet (4B10) & BioLegend & Cat. No. \#644804 \\
\hline PE/BV650 anti-mouse ROR $\gamma t$ (Q31-378) & $\mathrm{BD}$ & $\begin{array}{l}\text { Cat. No. \#562607 / } 564722 \\
\text { / } 562894\end{array}$ \\
\hline eFluor450 Eomes (Dan11mag) & ThemoFisher & Cat. No. \#48-4875-82 \\
\hline $\begin{array}{l}\text { AlexaFluor647 anti-mouse/human PLZF } \\
\text { (R17-809) }\end{array}$ & $\mathrm{BD}$ & Cat. No. \#563490 \\
\hline APC anti-human/mouse Bcl-6 (REA373) & Miltenyi & Cat. No. \#130-121-997 \\
\hline A700 anti-mouse Ki67 (16A8) & BioLegend & Cat. No. \#652420 \\
\hline anti-mouse CD16/32 (2.4G2) & In house & $\mathrm{N} / \mathrm{A}$ \\
\hline Fixable Viability Dye (LD) & ThermoFisher & Cat. No. \#65-0865-18 \\
\hline transcription factor staining buffer set & ThermoFisher & Cat. No. $\# 00-5523-00$ \\
\hline para-formaldehyde & EMS & Cat. No. \#50-980-488 \\
\hline LSR Fortessa & $\mathrm{BD}$ & $\begin{array}{l}\text { https://www.bdbiosciences. } \\
\text { com/en- } \\
\text { eu/instruments/research- } \\
\text { instruments/research-cell- } \\
\text { analyzers/Isrfortessa }\end{array}$ \\
\hline BD FACSymphony flow cytometer & $\mathrm{BD}$ & $\begin{array}{l}\text { https://www.bdbiosciences. } \\
\text { com/en- } \\
\text { eu/instruments/research- } \\
\text { instruments/research-cell- } \\
\text { analyzers/facsymphony }\end{array}$ \\
\hline FlowJo Software v10 & FlowJo & $\begin{array}{l}\text { https://www.flowjo.com/sol } \\
\text { utions/flowjo }\end{array}$ \\
\hline
\end{tabular}




\subsection{In vitro cell culture}

Single-cell suspensions from E14.5 fetal liver were enriched for $\mathrm{CD}^{+} 5^{+}$cells using magnetic activated cell sorting, followed by staining with fluorochrome-conjugated antibodies and sorted on BD FACSAria II (BD Bioscience) as live Lin $^{-} C D 45^{+}$Flt $3{ }^{-} C D 127^{+} \alpha_{4} \beta 7^{+}$PD $1^{-}$cells. Sorted lymphocytes were subsequently resuspended in IMDM (ThermoFisher) supplemented with $10 \%$ FCS, $100 \mathrm{U} / \mathrm{mL}$ penicillin, $0.1 \mathrm{mg} / \mathrm{mL}$ streptomycin, $50 \mu \mathrm{M} \beta$-mercaptoethanol (ThermoFisher) and each $20 \mathrm{ng} / \mathrm{mL}$ recombinant mouse IL-7 and SCF. Cells were plated onto 70\% confluent OP9 stromal cells in round bottom 96-well plates and medium was renewed every three days by replacing half of the media. Cells were analysed by flow cytometry after 5-8 days of culture.

Table 6. In vitro cell culture reagents.

\begin{tabular}{|c|c|c|}
\hline Reagent or resource & Source & Identifier \\
\hline OP9 & ATCC & CRL2749 \\
\hline CD45 microbeads & Miltenyi & Cat. No. \#130-052-301 \\
\hline IMDM & ThermoFisher & Cat. No. \#12440053 \\
\hline Penicillin-Streptomycin & ThermoFisher & Cat. No. \# 15140148 \\
\hline$\beta$-mercaptoethanol & ThermoFisher & Cat. No. \#21985023 \\
\hline IL-7, research grade & Miltenyi & Cat. No. \#130-094-066 \\
\hline SCF, research grade & Miltenyi & Cat. No. \#130-094-079 \\
\hline $\begin{array}{l}96 \text { well cell culture plate, } \\
\text { U-bottom }\end{array}$ & Greiner bio-one & Cat. No. \#650180 \\
\hline $\mathrm{CO}_{2}$ incubator & Binder & Cat. No. \#9040-0039 \\
\hline BD FACSAriaII cell sorter & $\mathrm{BD}$ & $\begin{array}{l}\text { https://www.bdbiosciences.com/en- } \\
\text { us/products/instruments/flow- } \\
\text { cytometers/research-cell- } \\
\text { sorters/bd-facsaria-iii }\end{array}$ \\
\hline
\end{tabular}

\subsection{Adoptive transfer}

For adoptive transfer experiments, ag $^{-1 /}$ I/2 $\mathrm{rg}^{/-} \mathrm{CD} 45.1$ recipient mice (Thy1.2/1.2) were sublethally irradiated with 5 Gy and endogenous, irradiation-resistant ILC3 populations were 
furthermore depleted by i.p. administration of $250 \mu \mathrm{g}$ anti-mouse Thy1.2 (30H12, in-house production) every third day. E14.5 FL lymphoid progenitors were FACS-sorted on an AriaII (BD Biosciences) instrument using the following sorting strategy LinLD $C D 45^{+} \mathrm{CD} 127^{+}$. CD $45.2^{+}$donor cells (Thy1.1/1.1 congenic) were injected i.v. (Figure 8). Mice were kept under antibiotic treatment in the first two weeks and analyzed 4 weeks after transfer.

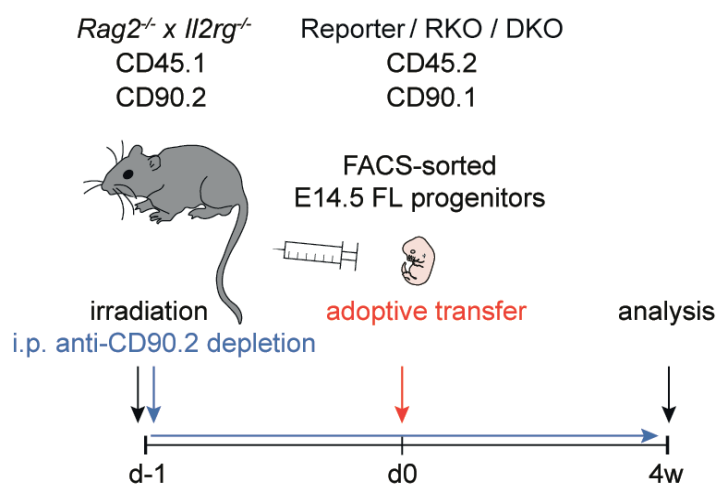

Figure 8. Schematic overview of adoptive transfer experimental setup. CD $45.1^{+} \mathrm{CD} 90.2^{+}$ congenic $\mathrm{Rag}^{-/} \mathrm{I}_{2} \mathrm{rg}^{--}$hosts were irradiated and additionally anti-CD90-depleted every third day from d-1 onwards. E14.5 fetal liver(FL)-derived progenitors of $\mathrm{CD} 45.2^{+} \mathrm{CD} 90.1^{+}$donor reporter (Rorc(gt)GFP/wt), RKO (Rorc(gt) $)^{G F / G F P}$ ) and DKO (Rorc(gt) ${ }^{G F P / G F P} x$ $\left.T b \times 21^{--}\right)$mouse strains were i.v. injected and host mice were analysed 4 weeks after reconstitution.

\subsection{Lymph node enumeration}

Percentages of mice with LN were calculated for each LN type using the total number of mice with the respective LN found in a given strain relative to the maximal number of mice with LNs of that type found in $\operatorname{Rorc}(g t)^{G F P / W t}$ or I/7rere control mice.

\subsection{Immunofluorescence staining}

Lymph nodes were fixed for $4 \mathrm{~h}$ in $4 \%$ paraformaldehyde and dehydrated via a sucrose gradient for $24 \mathrm{~h}$, embedded in O.C.T. compound and frozen in 2-Methylbutane within ethanol rinsed dry ice bath. Frozen lymph nodes were cryotome-cut into $7 \mu \mathrm{m}$ sections and placed on microscope slides in pairs. After thawing and PBS-rehydration of slides, primary staining was carried out after 20 min blocking with 10\% goat serum in PBS/BSA-0.1\%Tween20 (P-B-T). DAPI, anti-IgD-AF594, PNA-bio and rat-anti-mouse-CD35 were incubated for $1 \mathrm{~h}$ in P-B-T. Secondary staining was performed after washing in P-B-T by 30 min incubation with SA-A555 and anti-rat-IgG-A647 in P-B-T. Samples were finally washed in PBS and covered with mounting medium and cover slip and before image acquisition was performed on Zeiss LSM 880 confocal microscope. 
Table 7. Histology resources.

\begin{tabular}{|c|c|c|}
\hline Reagent or resource & Source & Identifier \\
\hline 2-Methylbutane & Carl Roth & Cat. No. \#3997.1 \\
\hline $\begin{array}{l}\text { 4',6-Diamidino-2-phenyl- } \\
\text { indol -dihydrochlorid }\end{array}$ & Sigma-Aldrich & Cat. No. \#D9542 \\
\hline $\begin{array}{l}\text { Anti-mouse-CD35 } \\
\text { unlabeled, rat IgG2a, kappa } \\
(8 \mathrm{C} 12)\end{array}$ & $\mathrm{BD}$ & Cat. No. \#558768 \\
\hline $\begin{array}{l}\text { Anti-rat-IgG-Alexa Fluor647, } \\
\text { Goat IgG polyclonal }\end{array}$ & ThermoFisher & Cat. No. \#A-21247 \\
\hline $\begin{array}{l}\text { Anti-mouse-IgD-Alexa } \\
\text { Fluor594, Rat IgG2a, kappa } \\
(11-26 c .2 a)\end{array}$ & BioLegend & Cat. No. \#405740 \\
\hline $\begin{array}{l}\text { Peanut-agglutinin, } \\
\text { biotynilated }\end{array}$ & Vector Labs & Cat. No. \#B-1075-5 \\
\hline Streptavidin-Alexa-Fluor555 & $\begin{array}{l}\text { Invitrogen by Thermo } \\
\text { Fisher Scientific }\end{array}$ & Cat. No. \#S32355 \\
\hline Ethanol & In-house & $\mathrm{N} / \mathrm{A}$ \\
\hline $\begin{array}{l}\text { Fluoromount Aqueous } \\
\text { Mounting Medium }\end{array}$ & Sigma-Aldrich & Cat. No. \#F4680 \\
\hline Paraformaldehyde & $\begin{array}{l}\text { Electron Microscopy } \\
\text { Sciences }\end{array}$ & Cat. No. \#15713 \\
\hline $\begin{array}{l}\text { Tissue-Tek O.C.T. } \\
\text { compound }\end{array}$ & Sakura & Cat. No. \#4583 \\
\hline Goat serum & Gibco & Cat. No. \#16210-064 \\
\hline
\end{tabular}

\subsection{Quantitative real-time PCR}

mRNA was isolated from small intestinal intraepithelial cells by using NucleoSpin ${ }^{\circledR}$ RNA Mini Kit (Macherey-Nagel) and following the manufacturer's protocol. RNA integrity was assessed on a 
BioAnalyzer (Agilent) and RNA samples with a RIN score $\geq 7$ were further transcribed into first strand cDNA by using Reverse Transcription Reagents (Applied Biosystems). qPCR reactions were assayed in triplicates per sample by using a StepOne Plus real-time PCR system and TaqMan Gene expression assays (all Thermofisher): Reg3g (Mm00441127_m1), Reg3b (Mm00440616_g1), Gapdh (Mm99999915_g1), HPRT (Mm03024075_m1) and Actb (Mm02619580_g1). mRNA content was normalized relative to the mean expression of the arithmetic means of Gapdh, Hprt and Actb CT values by applying the comparative $\mathrm{C}_{\mathrm{T}}$ method (2 ${ }^{\Delta C T}$ ) in which $\Delta C_{T}$ (gene of interest) $=C_{T}$ (gene of interest) $-C_{T}$ (arithmethic mean of housekeeping reference value).

Table 8. Commercial qPCR reagents.

\begin{tabular}{|c|c|c|}
\hline Reagent or resource & Source & Identifier \\
\hline NucleoSpin $尺$ RNA Mini Kit & Macherey-Nagel & Cat. No. \#740955.50 \\
\hline $\begin{array}{l}\text { TaqMan }^{\mathrm{TM}} \text { Reverse } \\
\text { Transcription Reagents }\end{array}$ & Invitrogen & Cat. No \#N8080234 \\
\hline $\begin{array}{l}\text { TaqMan Assay FAM-MGB: } \\
\text { Reg3g }\end{array}$ & ThermoFisher & Assay ID \#Mm00441127_m1 \\
\hline $\begin{array}{l}\text { TaqMan Assay FAM-MGB: } \\
\text { Reg3b }\end{array}$ & ThermoFisher & Assay ID \#Mm00440616_g1 \\
\hline $\begin{array}{l}\text { TaqMan Assay FAM-MGB: } \\
\text { Gapdh }\end{array}$ & ThermoFisher & Assay ID \#Mm99999915_g1 \\
\hline $\begin{array}{l}\text { TaqMan Assay FAM-MGB: } \\
\text { HPRT }\end{array}$ & ThermoFisher & Assay ID \#Mm03024075_m1 \\
\hline $\begin{array}{l}\text { TaqMan Assay FAM-MGB: } \\
\text { Actb }\end{array}$ & ThermoFisher & Assay ID \#Mm02619580_g1 \\
\hline BioAnalyzer 2100 & Agilent & Cat. No \#G2939BA \\
\hline $\begin{array}{l}\text { Bioanalyzer High Sensitivity } \\
\text { RNA } 6000 \text { Nano Kit }\end{array}$ & Agilent & Cat. No \#5067-1511 \\
\hline $\begin{array}{l}\text { StepOne Plus real-time PCR } \\
\text { system }\end{array}$ & ThermoFisher & Cat. No \#4376600 \\
\hline
\end{tabular}




\subsection{RNA sequencing and data analysis}

RNA of cells was extracted using RNeasy Plus Micro Kit (Qiagen) from sorted small intestinal lamina propria cells using the following markers LinLD'CD45 ${ }^{+} \mathrm{CD} 3^{-} \mathrm{ROR} \gamma \mathrm{t}-\mathrm{GFP}{ }^{+}$expressing either NKp46 or CD4. RNA quality was assessed by an Agilent 2100 Bioanalyzer. SMART-SeqII (ultralow input RNA) libraries were prepared using Nextera XT DNA sample preparation kit (Illumina). Sequencing was performed on an Illumina HiSeq4000 platform, PE100. For heatmaps, normalized RNA-seq data z-score transformed were plotted using ggplot2 R package. RNA-seq datasets analysed are publicly available in Gene Expression Omnibus repository with the accession number GSE161439.

Table 9. Bulk seq reagents.

\begin{tabular}{|l|l|l|}
\hline Reagent or resource & Source & Identifier \\
\hline RNeasy Plus Micro Kit & Qiagen & Cat. No \#74034 \\
\hline $\begin{array}{l}\text { Nextera XT DNA library } \\
\text { preparation kit }\end{array}$ & Illumina & Cat. No \#FC-131-1024 \\
\hline BioAnalyzer 2100 & Agilent & Cat. No \#G2939BA \\
\hline $\begin{array}{l}\text { Bioanalyzer High Sensitivity } \\
\text { RNA 6000 Pico Kit }\end{array}$ & Agilent & Cat. No \#5067-1513 \\
\hline HiSeq4000 platform & Illumina & $\begin{array}{l}\text { https://www.illumina.com/systems/seque } \\
\text { ncing-platforms/hiseq-3000-4000.html }\end{array}$ \\
\hline
\end{tabular}

\subsection{0x Genomics Chromium single-cell RNA-sequencing}

Fetal small intestinal lymphocytes were isolated as described above and sorted as LinLD$\mathrm{CD} 45^{+} \mathrm{CD} 122^{+}$and/or $\mathrm{CD} 127^{+}$. A total of 7 embryos from one pregnant Rorc $(g t)^{G F P / w t}$ female, 18 embryos from two pregnant $\operatorname{Rorc}(g t)^{G F P / G F P}$ females and 19 embryos from three pregnant $\operatorname{Rorc}(g t)^{G F P / W t} \times T$ TbX21\%- females were used. Samples were furthermore labelled with antibodyderived tags against specific extra-cellular targets and genotypes hash-tagged with TotalSeq ${ }^{\mathrm{TM}}$ A antibodies (BioLegend) following the manufacturer's protocol. A total of 8000 cells were loaded in the Chromium ${ }^{\mathrm{TM}}$ Controller for partitioning single cells into nanoliter-scale Gel Bead-InEmulsions (GEMs). Single Cell 3' reagent kit v3.1 was used for reverse transcription, cDNA amplification and library construction of gene expression libraries (10x Genomics) according to the manufacturer's instructions. TotalSeq ${ }^{\mathrm{TM}}-\mathrm{A}$ libraries were prepared following the protocol for 10x Single Cell 3' Reagent Kit v3.1 provided by BioLegend, including primer sequences and 
reagent specifications. Concentrations of all libraries were quantified using a QubitTM 2.0 Fluorometer (ThermoFisher) and quality was tested on a 2100 Bioanalyzer with High Sensitivity DNA kit (Agilent). Sequencing was performed using the NextSeq500/550 HighOutput Kit v2.5 (150 cycles) on a NextSeq500 sequencer (Illumina). scRNA-seq datasets analyzed are publicly available in Gene Expression Omnibus repository with the accession number GSE161441.

Table 10. ScRNA-seq reagents.

\begin{tabular}{|c|c|c|}
\hline Reagent or resource & Source & Identifier \\
\hline Anti-mouse CD49b (HMa2) & BioLegend & Cat. No. \#103523 \\
\hline Anti-mouse NK1.1 (PK136) & BioLegend & Cat. No. \#108755 \\
\hline Anti-mouse DNAM1 (10E5) & BioLegend & Cat. No. \#128823 \\
\hline Anti-mouse CD3 (17A2) & BioLegend & Cat. No. \#100251 \\
\hline Anti-mouse CCR6 (29-2L17) & BioLegend & Cat. No. \#129825 \\
\hline Anti-mouse NKp46 (29A1.4) & BioLegend & Cat. No. \#137633 \\
\hline Anti-mouse CD117 (2B8) & BioLegend & Cat. No. \#105843 \\
\hline Anti-mouse Nrp-1 (3E12) & BioLegend & Cat. No. \#145215 \\
\hline Anti-mouse NKG2D $\quad(C X 5)$ & BioLegend & Cat. No. \#130215 \\
\hline Anti-mouse TCRgd (GL3) & BioLegend & Cat. No. \#118137 \\
\hline Anti-mouse TCRb (H57-597) & BioLegend & Cat. No. \#109247 \\
\hline Anti-mouse CD49a (HMa1) & BioLegend & Cat. No. \#142613 \\
\hline Anti-mouse CXCR5 (L138D7) & BioLegend & Cat. No. \#145535 \\
\hline Anti-mouse CD27 (LG.3A10) & BioLegend & Cat. No. \#124235 \\
\hline Anti-mouse CD200R1 (OX-110) & BioLegend & Cat. No. \#123913 \\
\hline Anti-mouse CD4 (RM4-5) & BioLegend & Cat. No. \#100569 \\
\hline Anti-mouse PD-1 (RMP1-30) & BioLegend & Cat. No. \#109123 \\
\hline Anti-PE(PE001) & BioLegend & Cat. No. \#408109 \\
\hline anti-Biotin (1D4-C5) & BioLegend & Cat. No. \#409008 \\
\hline
\end{tabular}




\begin{tabular}{|c|c|c|}
\hline Anti-mouse ICOS (C398.4A) & BioLegend & Cat. No. \#313555 \\
\hline Anti-mouse Sca-1 (D7) & BioLegend & Cat. No. \#108147 \\
\hline Anti-mouse ST2 (DIH9) & BioLegend & Cat. No. \#145317 \\
\hline Anti-mouse CD25 (PC61) & BioLegend & Cat. No. \#102055 \\
\hline Anti-mouse KLRG1 (2F1/KLRG1) & BioLegend & Cat. No. \#138431 \\
\hline Hashtag A0301 (M1/42; 30-F11) & BioLegend & Cat. No. \#155801 \\
\hline Hashtag A0302 (M1/42; 30-F11) & BioLegend & Cat. No. \#155803 \\
\hline Hashtag A0303 (M1/42; 30-F11) & BioLegend & Cat. No. \#155805 \\
\hline Single Cell $3^{\prime}$ reagent kit v3.1 & 10x Genomics & Cat. No. \# PN-1000121 \\
\hline Chromium $^{\mathrm{TM}}$ Controller & 10x Genomics & $\begin{array}{l}\text { https://www.10xgenomics.co } \\
\text { m/instruments/chromium- } \\
\text { controller }\end{array}$ \\
\hline Qubit ${ }^{\mathrm{TM}}$ 2.0 Fluorometer & ThermoFisher & $N / A$ \\
\hline Qubit ${ }^{T M}$ dsDNA HS Assay Kit & ThermoFisher & Cat. No \#Q32854 \\
\hline BioAnalyzer 2100 & Agilent & Cat. No \#G2939BA \\
\hline $\begin{array}{l}\text { Bioanalyzer High Sensitivity DNA } \\
\text { Analysis }\end{array}$ & Agilent & Cat. No \#5067-4626 \\
\hline NextSeq500/550 HighOutput Kit v2.5 & Illumina & Cat. No \#20024907 \\
\hline NextSeq500 sequencer & Illumina & $\mathrm{N} / \mathrm{A}$ \\
\hline
\end{tabular}

\subsection{1 scRNA-seq data processing}

Demultiplexing and alignment to the mm10 reference transcriptome was performed with Cell Ranger v3.0.2 (Zheng et al., 2017). Antibody- and hashtag-barcodes were counted with CITEseq-Count (Roelli et al., 2019) and normalized by centered log ratio transformation with Seurat v3.0.2 (Stuart et al., 2019), which was also used for further data processing. Genotypes were assigned based on normalized hashtag counts. Cells were filtered by number of transcripts, genes, percent mitochondrial genes and for cells present within transcriptome, antibody and hashtag libraries. Normalized expression values were generated with scran (Lun et al., 2016), 
followed by scaling and principal component (PC) analysis. PC loadings and explained variance were visualized to determine PCs used for dimensionality reduction by uniform manifold approximation (UMAP) (McInnes et al., 2018) and Seurat clustering. Wilcoxon test was applied for differential gene expression analysis of genes expressed by $\geq 10 \%$ of cells in a cluster and with a log fold change of $\geq 0.25$. Lineage structures on UMAPs were analyzed with Slingshot (Street et al., 2018), giving the starting (CLP) and end populations (ILC1/2/3) as input. Partitionbased graph abstraction (PAGA) using the Fruchterman \& Reingold algorithm was performed within Scanpy v1.4.6 (Wolf et al., 2019) after import of pre-processed Seurat objects into Python with anndata2ri (A. et al., 2020). scRNA-seq data analysis was performed with R v4.0.0 and Python v3.7.6. Gene modules of specific subsets from cells of $\operatorname{Rorc}(g t)^{G F P / w t}$ mice were defined by the top 100 differentially regulates genes calculated from the comparison of all cells from all subsets.

Table 11. ScRNA-seq data processing algorithms.

\begin{tabular}{|l|l|l|}
\hline Reagent or resource & Source & Identifier \\
\hline Cell Ranger v3.0.2 & (Zheng et al., 2017) & $\begin{array}{l}\text { https://support.10xgenomics.com/singl } \\
\text { e-cell-gene- } \\
\text { expression/software/pipelines/latest/usi } \\
\text { ng/count }\end{array}$ \\
\hline CITE-seq-Count & (Roelli et al., 2019) & \\
\hline R Studio v4.0.0 & (Stuart et al., 2019) & https://rstudio.com/ \\
\hline Seurat v3.0.2 & (Lun et al., 2016) & \\
\hline Scran & (Street et al., 2018) & \\
\hline Slingshot & (Wolf et al., 2019) & \\
\hline Scanpy v1.4.6 & (A. et al., 2020) & https://zenodo.org/record/3992373 \\
\hline Python v3.7.6 & & \\
\hline Anndata2ri & & \\
\hline
\end{tabular}

\subsection{Statistical analysis}

Kruskal-Wallis test with Dunn's multiple comparison correction was employed for statistical analysis of datasets. Statistical analyses were performed with Prism 5.04 (GraphPad Software) 
using a confidence level of 0.95 , and $P$ values $>0.05$ were considered not significant, $P$ values from 0.01 to 0.05 were considered as $*$, $\mathrm{P}$ values from 0.001 to 0.005 were considered as $* *, \mathrm{P}$ values $<0.0001$ were considered as $* * *$.

Table 12. Softwares and algorithms.

\begin{tabular}{|l|l|l|}
\hline Reagent or resource & Source & Identifier \\
\hline GraphPadPrism 5.04 & GraphPad Software Inc & $\begin{array}{l}\text { https://www.graphpad.com/scientific- } \\
\text { software/prism/ }\end{array}$ \\
\hline FlowJo 10 & FlowJo LLC & https://www.flowjo.com/solutions/flowjo \\
\hline R Studio v4.0.0 & R Core Team & https://rstudio.com/ \\
\hline Python v3.7.6 & $\begin{array}{l}\text { Python Software } \\
\text { houndation }\end{array}$ & \\
\hline & https://www.python.org/ \\
\hline
\end{tabular}




\section{Results}

\subsection{Identification of fetal ILC subsets in the developing mouse intestine}

In order to study embryonic ILCs, the expression of the commonly used inclusion marker CD127 or IL-7R (Vonarbourg and Diefenbach, 2012) was characterized in the fetal small intestinal (SI) lamina propria 18.5 days post conception (E18.5). After exclusion of dead and lineage (CD19, FcERI $\alpha, \mathrm{GR}-1, \mathrm{~F} 4 / 80, \mathrm{CD} 3)^{+}$cells and inclusion of $\mathrm{CD}^{4} 5^{+}$cells of hematopoietic origin, CD127 was found to be highly expressed on RORyt ${ }^{+}$ILC3, lower on GATA3 ${ }^{\text {hi }}$ ILC2 and lowest on T-bet ${ }^{+}$ ILC1/NK. The usage of an additional marker CD122 (also known as IL-2R $\beta$ ), a member of the type I cytokine receptor family, that was highly expressed on T-bet ${ }^{+}$ILC1/NK enabled us to incorporate all members of the ILCs including T-bet-expressing ILC1 (Figure 9 A, B). Further validating the gating strategy, additional ILC markers CXCR6 as well as CD90 were also expressed by all subsets and can be used to study embryonic intestinal ILCs (Figure 9 B, C). Gating on CD122-CD127- cells revealed absence of CXCR6-, CD90- as well as ROR $\gamma$ t-, T-bet- and GATA3expressing cells, emphasizing the approach of using not only CD127 but also CD122 as valuable inclusion markers for ILC identification in the embryonic gut. 
A

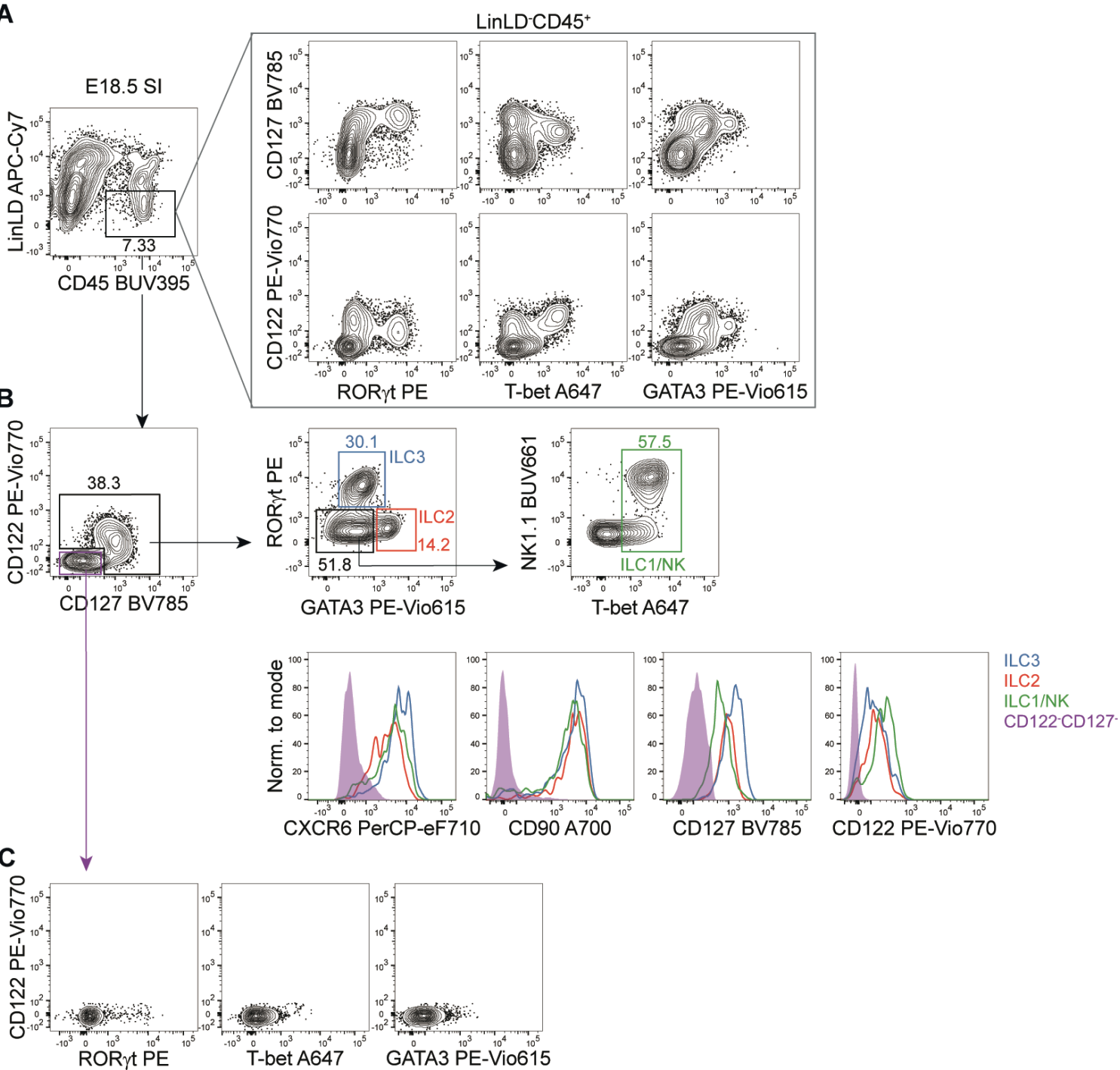

Figure 9. Identification of embryonic ILCs by flow cytometry. (A) Flow cytometric representative plots showing expression of transcription factors on CD127+ and/or CD122+ on LinLD-CD45+ cells of the E18.5 small intestine (SI). (B) Specific ILC populations are defined by transcription factors within CD127 $7^{+}$and/or CD122 ${ }^{+}$cells that furthermore express the surface markers CXCR6 or CD90. (C) Within CD127- CD122- minor populations of ROR $\gamma$ t, T-bet or GATA3 are detected.

\subsection{Single-cell sequencing of fetal intestinal cells reveals a spectrum of mature ILC subsets and progenitors}

For the purpose to study prenatal ILC heterogeneity, single-cell RNA sequencing (scRNA-seq) of viable Lin(CD19, CD3, CD5, F4/80,FCERI $\alpha, \mathrm{Gr}-1)^{-}$CD45+ cells expressing CD127 and/or CD122 isolated from the small intestine (SI) of embryonic day 18.5 (E18.5) of $\operatorname{Rorc}(g t)^{G F P / w t}$ mice was performed.

For simultaneous characterization of RNA and protein, oligo-conjugated antibodies were integrated into the single-cell sequencing workflow, also known as CITE-seq (Cellular Indexing of Transcriptomes and Epitopes by sequencing) that enabled validation of ILC lineage identities with surface protein expression. After quality control, normalization and filtering out a DC-like population (displaying transcripts for Cd209a, Cd86 and Cfs1r, Figure 10 A), we analysed 1268 
remaining cells. Clearly distinct gene signatures marked by 498-1466 differentially expressed genes (DEGs) led to the identification of eight distinct clusters (Figure 10 B and Figure $11 \mathbf{A}$ ).
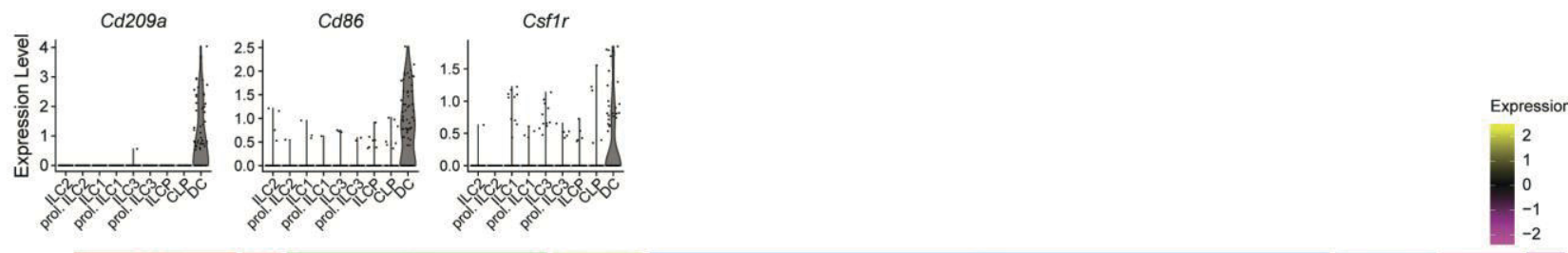

B

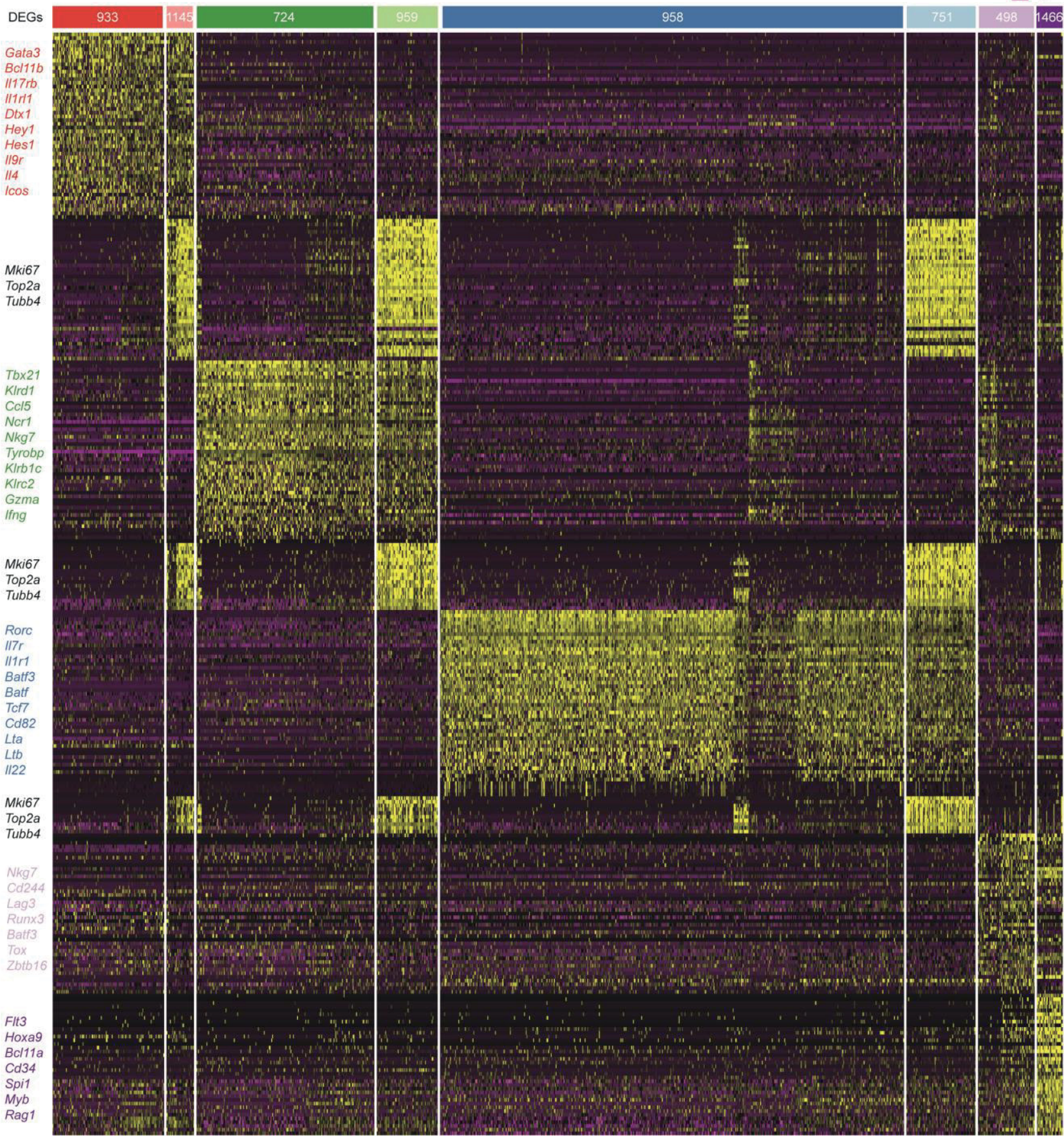

Figure 10. Single-cell RNA sequencing of E18.5 intestinal cells identifies distinct clusters. Viable Lin(CD19, CD3, CD5, F4/80, FceRIa, Gr-1)- CD45+ cells expressing IL-7 receptor (CD127) and/or the IL-2 receptor subunit beta (CD122) isolated from the small intestine (SI) of E18.5 Rorc(gt) ${ }^{G F P / w t}$ embryos were sort-purified by flow cytometry, and a single-cell expression library was generated using 10x Genomics. (A) Violin plots of selected dendritic cell (DC)associated markers. (B) Heatmap displaying the top 50 differentially expressed genes (DEGs) within each cluster, gene examples are given. 


\section{Results}

The key ILC marker Id2 was transcribed in the majority of cells assigning these cells to the ILC lineage, except for one small cluster 8 (Figure 11 B, D). Besides Id2, other fundamental ILC transcription factors like Ets1, Rora or Ahrtranscripts were detected (Figure $11 \mathrm{D}$ ). Strong gene signatures, including transcripts for Gata3, Tbx21 and Rorc, facilitated the identification of three major superclusters comprising of ILC2 (cluster 1 and 7), of ILC1/NK (hereafter referred to as ILC1, cluster 3 and 5) and of ILC3/LTi (hereafter referred to as ILC3, cluster 2 and 6) (Figure 11 A, B, D). Identity of the inferred superclusters was also confirmed by the distribution of surface markers detected by oligo-conjugated antibodies as well as flow cytometry, ST2, NK1.1 and CD4 (Figure 11 B, C). Top 50 differentially expressed genes between these clusters included BCl11b, I/1rl1, I/17rb, ICOS, Hey1, I/9r and I/4 for ILC2; CCI5, KIrk1, Ncr1, CXCr3, XCl1 and Ifng for ILC1; Batf3, Batf, Tox2, I/1r1, I/23r, Nrp1, Lta and I/22 for ILC3 (Figure 10 B and Figure 11 D) (Gury-BenAri et al., 2016; Pokrovskii et al., 2019; Robinette et al., 2015). As also observed on protein level, transcripts for I/7r were highest in ILC3 and lowest on ILC1/NK, which conversely showcased most abundant I/2rb transcripts (Figure 11 B, D). Within all ILC superclusters, we further detected clusters of cells (5-7) enriched in gene transcripts associated to active cell cycle, such as Mki67, Top2a and Tubb4, highlighting a substantial fraction of proliferating ILC3, ILC2 and ILC1 within the embryonic intestine (Figure 10 B and Figure 11 A). Expression of the NK cell TF Eomes was mostly negative and limited to a small subset within Tbx $21^{+}$cells, supporting the notion that clusters 3 and 5 mainly comprise of ILC1 (Figure 11 D, E). In addition to classical ILC lineages, two other clusters (4 and 8) were observed of which cluster 8 lacked expression of most ILC-related genes such as Id 2 but displayed abundant transcripts for F/t3, Cd34 and BCl11a along with $\operatorname{Il} 7 \mathrm{r}$ (Figure 11 B, D). Moreover, transcripts for Tcf3 (encoding for E2A) which facilitates lymphocyte differentiation from hematopoietic stem cells but also Nfil3 transcripts were found, a transcription factor that controls NK/ILC lineage commitment by regulating Id2 expression (Male et al., 2014; Seillet et al., 2014b, 2014a; Xu et al., 2015). Few cells within this cluster additionally showcased expression of recombination activating genes Rag1 or Early B cell Factor 1 (Ebf1), altogether designating this expression signature being compatible with the one previously described for common lymphocyte progenitors (CLP) from adult BM (Ghaedi et al., 2016). Cluster 4 on the other hand showed high expression of most ILC lineage-associated genes such as Id2, Ets, Rora or Gata3 but also had abundant transcripts for Zbtb16 encoding for PLZF, Tcf7 or Tox, core regulators of multiple ILC developmental pathways (Constantinides et al., 2014; Klose et al., 2014; Mielke et al., 2013; Seillet et al., 2016; Yang et al., 2013). Transcripts such as Ikzf2, Runx3 and Maf, genes that are implied in commitment to ILC lineages were similarly upregulated in this cluster suggesting that these cells are enriched in ILC precursors (ILCP), sharing a gene signature previously described in FL and adult BM (Figure 11 D). Thus, cluster 8 and 4 are hereafter referred to as CLP and ILCP, respectively. Interestingly, a conversion from Notch1 to Notch2 expression was noted in 
these two progenitor clusters underlining the transition from CLP to ILCP as reported in FL progenitors by Golub et al. (Chea et al., 2016b).

A

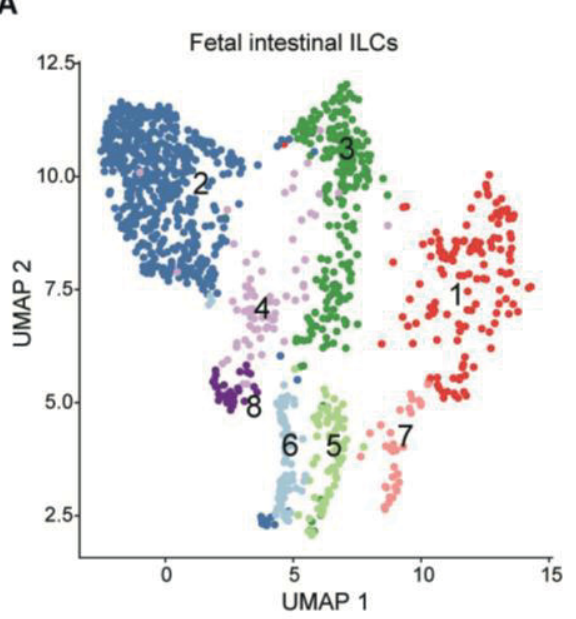

B Id2

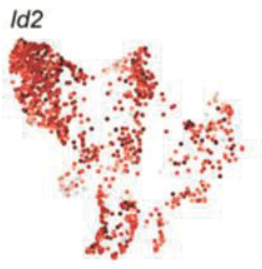

Rorc

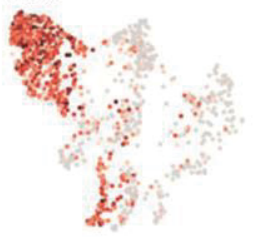

protein $\mathrm{CD} 4$

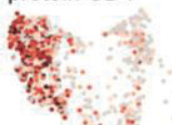

3.

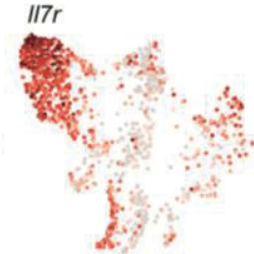

Tbx21
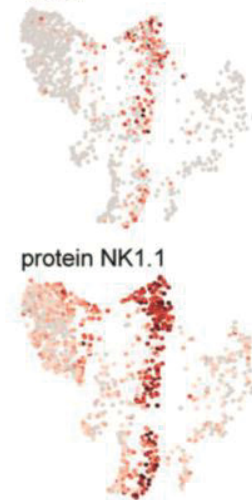

- ILC2

- prol. ILC2

- ILC1

- prol. ILC1

- ILC3

- prol. ILC3

- CLP

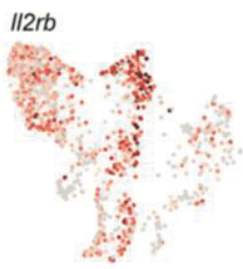

Gata3

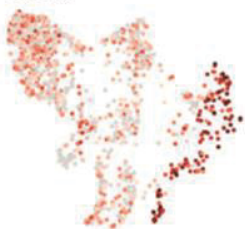

protein ST2

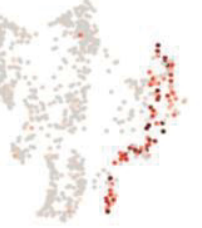

C LinLDCD $45^{+}$

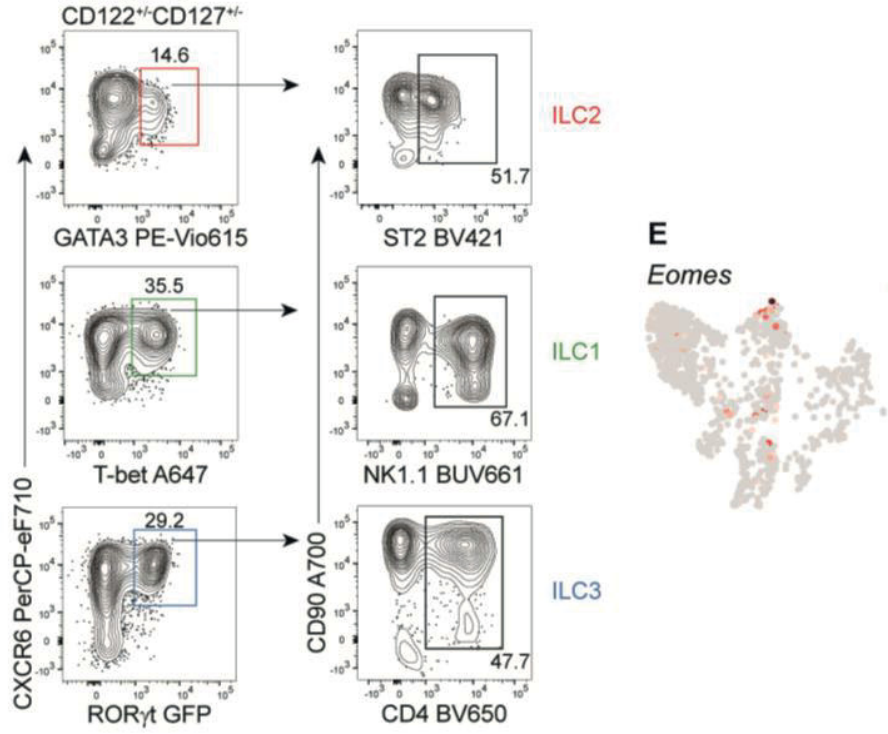

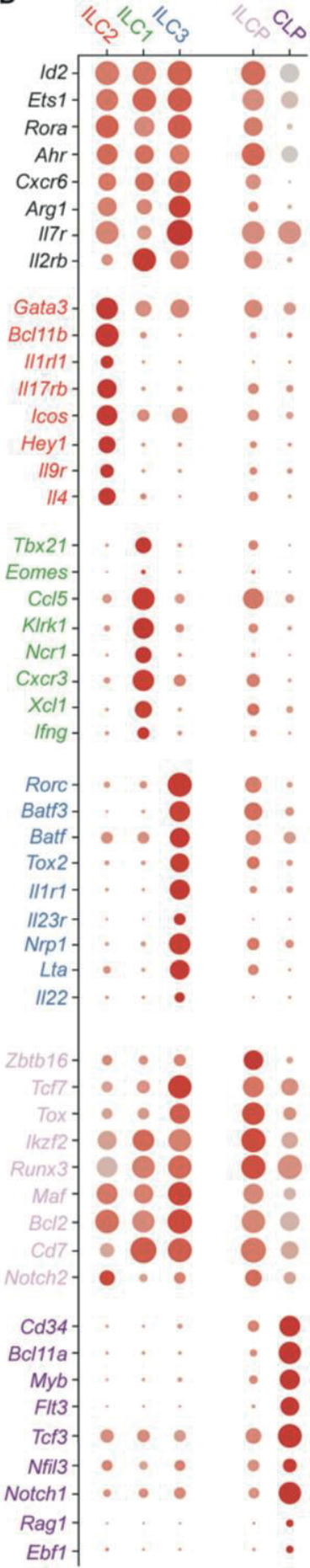

Average expression Percent expressed

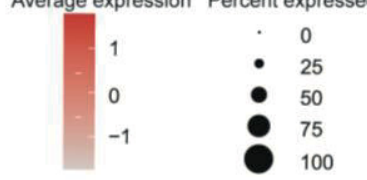

*legend on next page 


\section{Results}

Figure 11. Single-cell RNA sequencing of fetal intestinal cells reveals a spectrum of mature ILC subsets and progenitors. Viable Lin(CD19, CD3, CD5, F4/80, FceRIa, Gr-1)- CD45+ cells expressing IL-7 receptor (CD127) and/or the IL-2 receptor subunit beta (CD122) isolated from the small intestine (SI) of E18.5 Rorc(gt) ${ }^{G F P / w t}$ embryos were sort-purified by flow cytometry, and a single-cell expression library was generated using 10x Genomics. (A) UMAP dimensional reduction projection identifies 8 distinct clusters. (B) Gene expression and CITE-seq protein expression UMAP plots. $(\mathbf{C})$ representative flow cytometry plots identifying ST2 expression on GATA3 ${ }^{+}$ILC2, NK1.1 experssion on T-bet ${ }^{+}$NK/ILC1 and CD4 expression by ROR $\gamma$ t-GFP+ ILC3. (D) Selected gene expression within clusters. Colour scale represents average expression, dot size visualizes fraction of cells within the cluster expressing the gene. (E) UMAP-projected expression of Eomes transcripts.

ILCP mostly co-expressed Zbtb16 together with Tcf7, Tox and also Gata3'o, while few cells coexpressed Zbtb16 with CXCr6, Gata3hi, Tbx21 or Rorc with only little or no expression in CLP (Figure 12 A), suggestive of continuous transition from ILCP towards the diverse ILC lineages. On this matter, expression of genes linked to specific lineages such as ILC1-associated transcripts CXCr3, KIrk1, XCl1, as well as ILC2-associated transcripts I/17rb, Icos, I/4, and ILC3-associated transcripts Batf3, Batf, Nrp1 and I/1r1 could be observed accordingly within the ILCP cluster (Figure 11 D). In line with this, surface PD-1 expression, which was shown to identify PLZF+ ILCP in BM (Seillet et al., 2016; Yu et al., 2016) correlated with Zbtb16 transcripts. Fetal intestinal ILCP were also characterized by the expression of the heterodimeric integrin receptor $\alpha_{4} \beta_{7}$ and c-Kit, as previously shown for their BM and FL counterpart (Figure 12 B).

In order to test whether the transcriptional signatures displayed by fetal intestinal ILC and progenitors were compatible with a model of in situ differentiation from CLP, via ILCP, to mature ILC subsets single-cell developmental trajectories were inferred, using two distinct computational models, namely partition-based graph abstraction (PAGA) (Figure 12 C) (Wolf et al., 2019) and Slingshot (Figure 12 D) (Street et al., 2018). For this analysis, cell-cycle related genes were regressed out to correct the positioning of the proliferating clusters $(5,6,7)$ in the embedding, thereby mapping these cells onto their respective ILC superclusters. Both analyses revealed comparable trajectories with continuous cell transitions from CLP to ILCP, further branching into ILC1, ILC2 and ILC3. Of note, Slingshot analysis emphasized an early branching of the ILCP towards the ILC2 cluster, while ILC1 and ILC3 retained a common trajectory and transitional states before bifurcating into separate lineages (Figure 12 D). Pseudotime analysis of selected genes showed early expression of Zbtb16, Tcf7 and Tox where ILC1/NK upregulate Zbtb16 and ILC3 upregulate TCf7 after lineage commitment (Figure 12 E). Notably, ILC3 lineage shows lowest expression of $Z b t b 16$ but highest expression of Tcf7 suggesting a potential regulation of ILC3 differentiation by these two TFs. Additional evaluation of I/1rl1, Ncr1 and I/1r1 transcripts in ILC2, ILC1 and ILC3 lineages respectively indicated upregulation of these genes later in pseudotime and validated the analysis (Figure $12 \mathrm{E}$ ). 
A
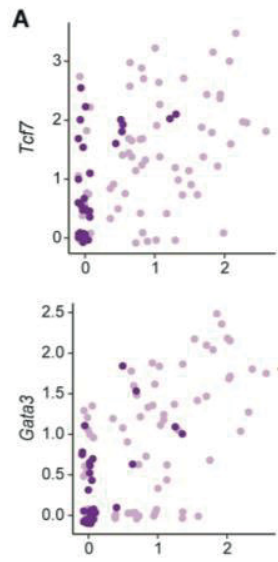

C

PAGA trajectory analysis

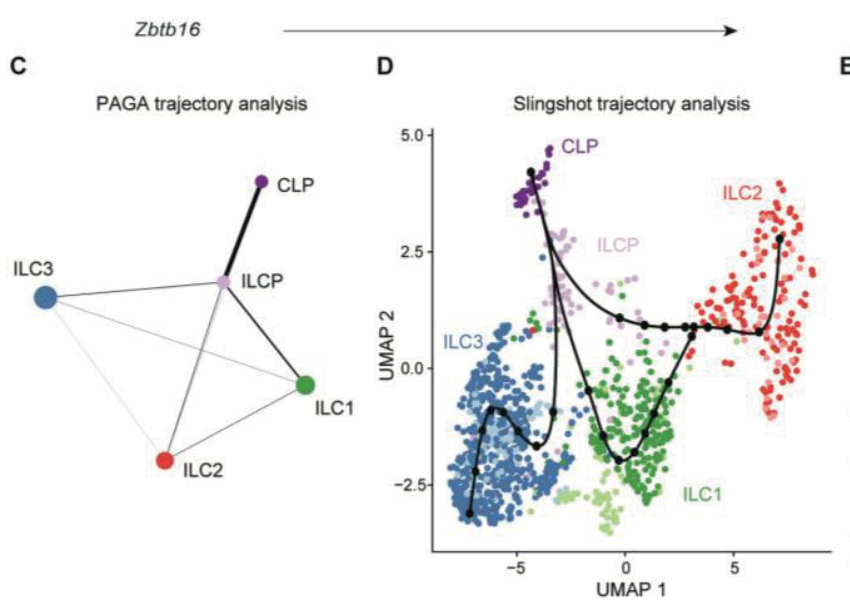

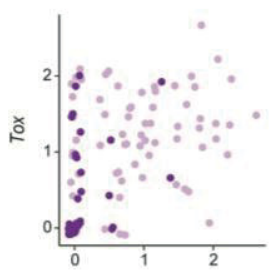
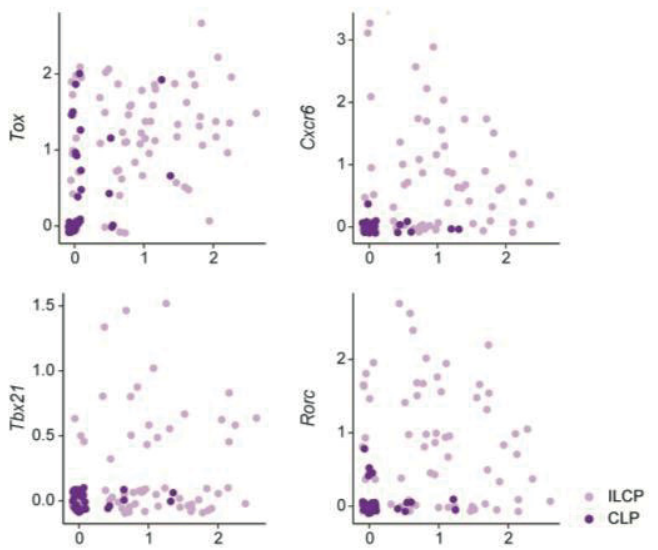
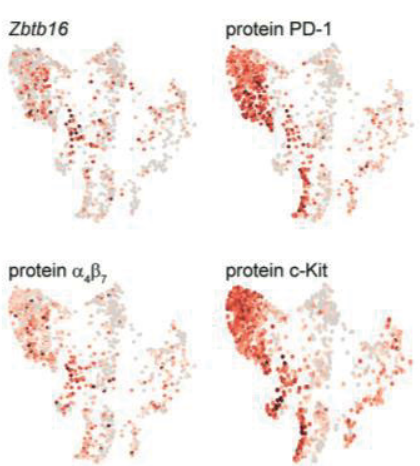

E ILC1 ILC2 ILC3
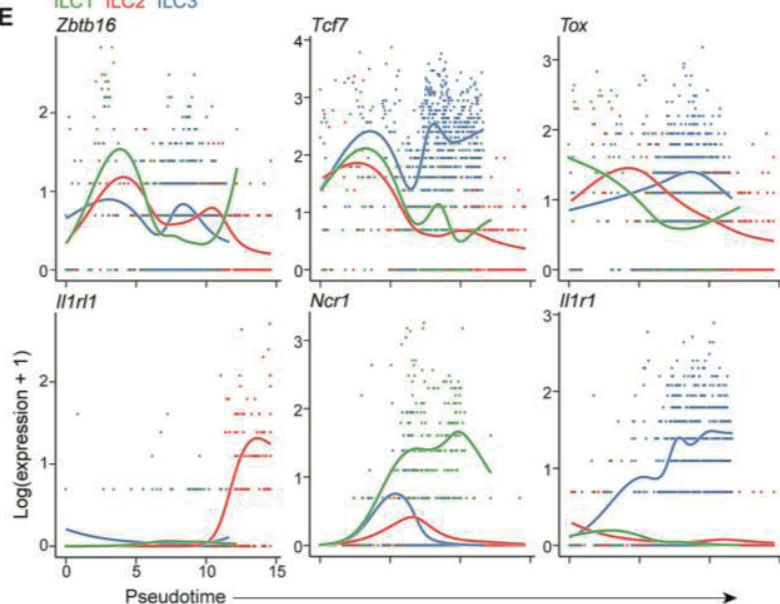

Figure 12. Single-cell RNA sequencing defines CLP and ILCP progenitors within the embryonic intestine. (A) Zbtb16 co-expression plots of selected genes within CLP and ILCP cluster. (B) Gene expression and CITE-seq protein expression UMAP plots. (C) Trajectory analysis using PAGA lineage interference method. (D) Trajectory analysis using Slingshot. Inferred trajectories are represented as lines starting within the CLP cluster. Dots represent knots. (E) Selected gene expression over pseudotime within ILC1, ILC2 and ILC3.

Altogether, these data report prenatal transcriptional signature of ILC lineages in SI lamina propria and identify progenitor populations within the isolated tissue that resemble those described in FL or adult BM, highlighting the fetal intestine as an active niche for ILC differentiation.

\subsection{Transcriptomic profiling on single-cell level discloses heterogeneity within the embryonic ILC3 supercluster}

Next, the ILC3 cluster was further dissected to assess whether fetal ILC3 comprise heterogeneous subsets similar to their adult counterparts. Using unsupervised clustering, three subclusters with defined sets of 350-496 differentially regulated genes were identified (Figure 13 A and B), highlighting heterogeneity within this fetal ILC lineage, despite comparable expression of the ILC3 TF Rorc in all three subclusters (Figure $13 \mathrm{C}$ ). Among the top 30 genes characterizing subcluster 1, Tnfsf11 (encoding RANKL or TRANCE), Pdcd1 and transcripts for 
cytokines or chemokines such as I/22, Lif(Cella et al., 2009), Csf2 (encoding GM-CSF) and the neutrophil chemoattractant $C X C / 2$ (Figure 13 C, D) were found. IITr, Ltb, Ccr6, Batf and Cd7 were among the transcripts enriched in subcluster 2. Interestingly, expression of transcripts associated with major histocompatibility (MHC) class molecules, including $\mathrm{H} 2$-genes as well as Cd74, Cd82 and Lst1, previously reported in CD4 ${ }^{+}$fetal LTi cells and in CCR6 ${ }^{+}$ILC3 postnatally (Von Burg et al., 2014; Hepworth et al., 2013; Lehmann et al., 2020; Mackley et al., 2015; Mebius et al., 1997; Robinette et al., 2015), were specifically upregulated in this subcluster (Figure 13 B). Subcluster 3 showcased augmented expression of genes typically associated to T-betexpressing ILC1 and NK cell subsets, as well as adult NKp46+ ILC3, such as KIrk1, KIrC2, KIrd1, KIrb1C together with $\mathrm{Nkg} 7$ (Figure $13 \mathrm{C}, \mathrm{D}$ ). Overall, these data suggest that generation of specified ILC3 subsets already happens before birth. Therefore, selected gene and protein expression patterns indicative of T-bet expression were analyzed in the next step to find out whether these cells could potentially be distinguished by surface markers. 
A

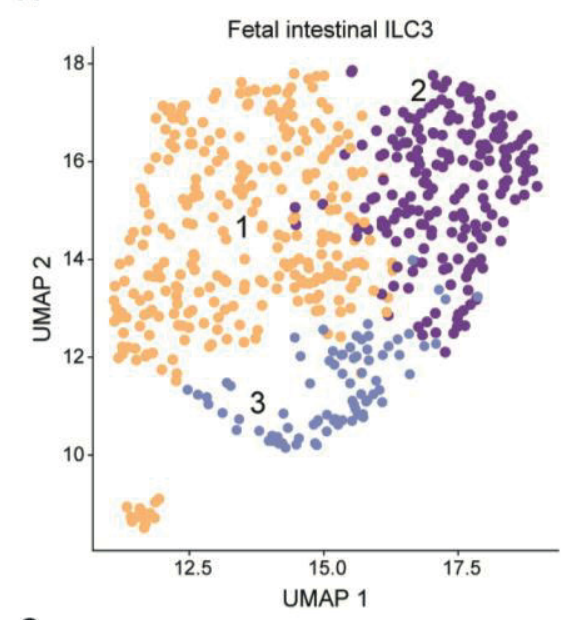

C

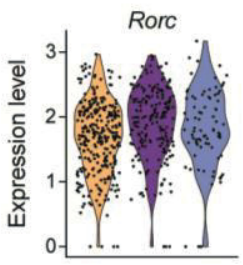

D $\quad 1122$

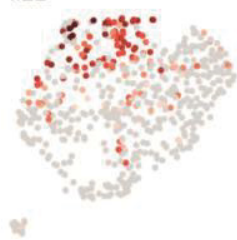

Ccr6

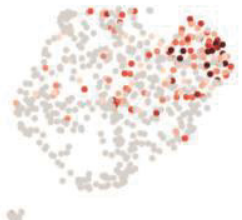

KIrk1

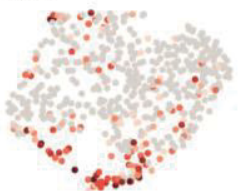

B

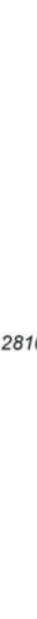

op 30 DEGs within ILC3 subclusters

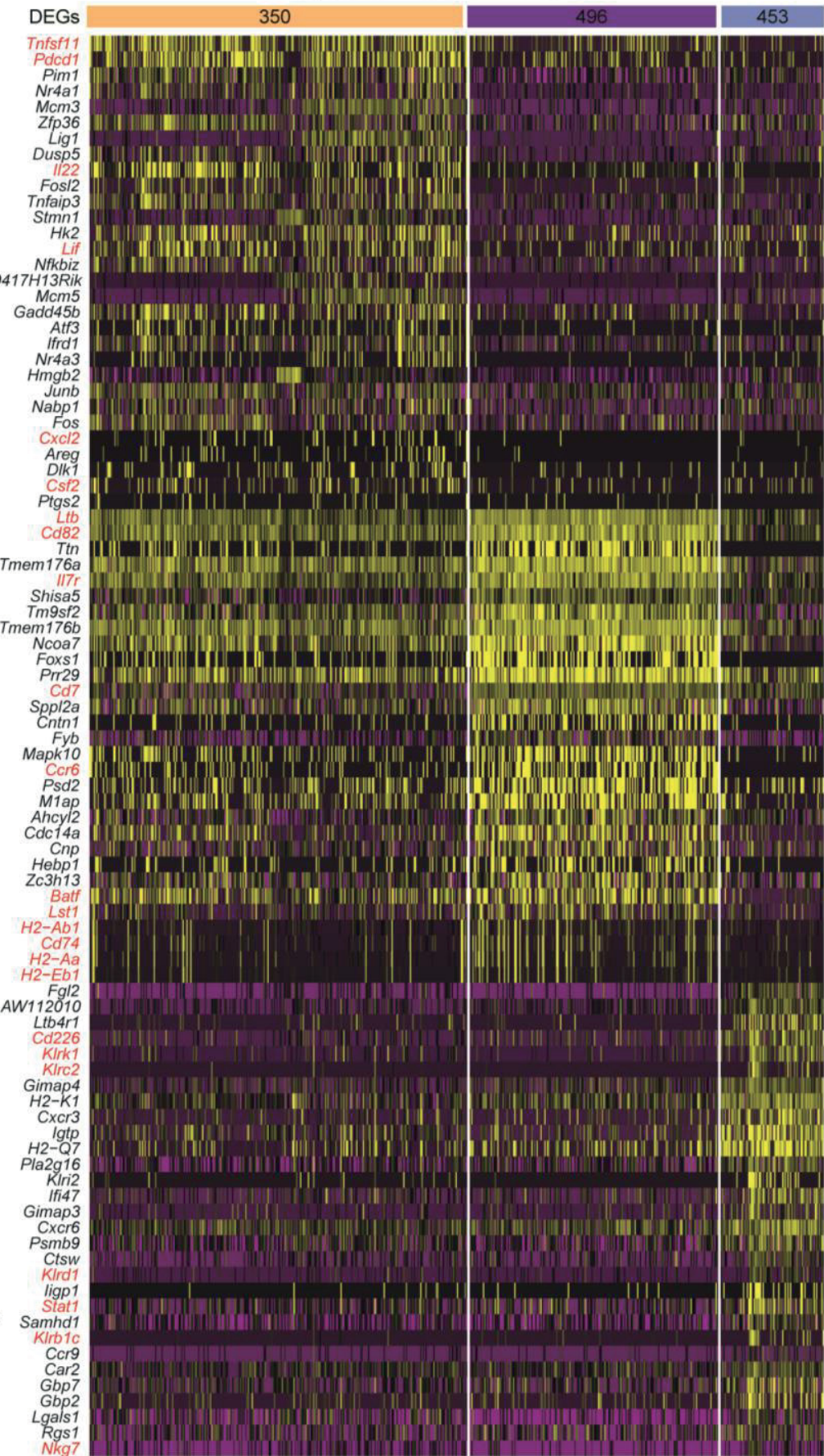

Figure 13. Gene expression patterns of specified embryonic ILC3 subsets defined by SCRNA-seq. (A) UMAP dimensional reduction projection reveals three separate subclusters within the ILC3 cluster. (B) Heatmap displaying the top 30 differentially expressed genes (DEGs). (C) Violin plot of Rorc expression within ILC3 subclusters. (D) Selected gene expression UMAP plots.

Indeed, cell surface stainings of c-Kit and DNAM-1 protein correlated with enriched transcripts and displayed differential surface binding (Figure 14 A). Hence, these markers could be used to characterize prenatal ILC3 subsets similar to how they were applied to differentiate between subsets in adult mice (Verrier et al., 2016). Besides aforementioned enriched transcripts of LTiassociated gene Tnfsf11 in subcluster 1 , also $\mathrm{CXCr} 5$ and $L$ Ta were among the DEGs (Figure 14 B) suggesting that this subset represents cells capable of inducing LN formation. Importantly, 


\section{Results}

protein expression of CD4 or NRP-1, surface receptors previously reported to identify LTi cells in embryos or adult mice and humans (Robinette et al., 2015; Shikhagaie et al., 2017), did not resolve cluster 1 and 2 (Figure 14 B). Additionally, cells expressing transcripts for Tbx21 as well as for Ncr1 (encoding for NKp46) were detected (Figure 14 C) and enriched in subcluster 3. In line with low expression of CD127 protein observed in T-bet-expressing ILCs (Figure 14 D), II7rtranscripts as well as CD127 protein were lowest in this subcluster of ILC3 co-expressing T-bet (Figure 9 A and B).

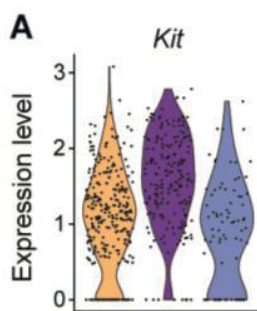

Cd226

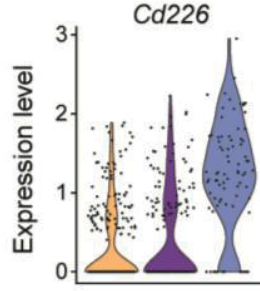

C

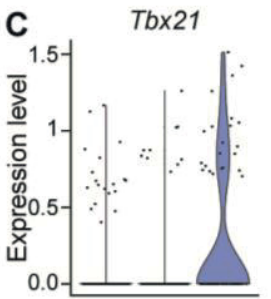

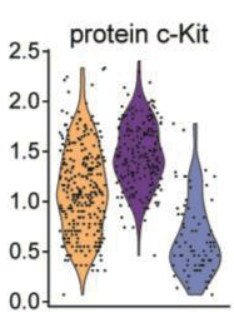

protein DNAM-1

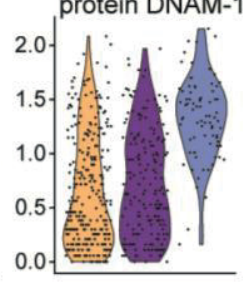

$\mathrm{Nor1}$

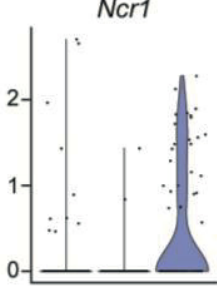

B

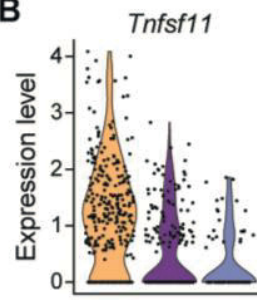

protein CD4

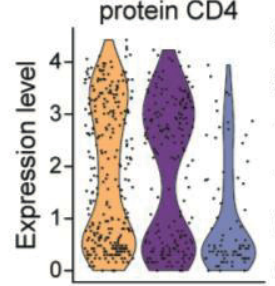

D

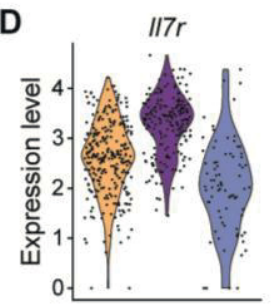

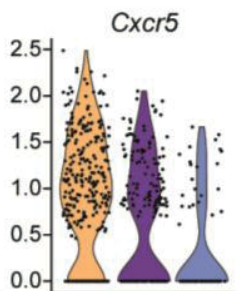

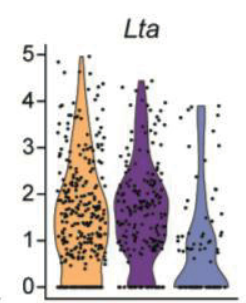

protein NRP-1
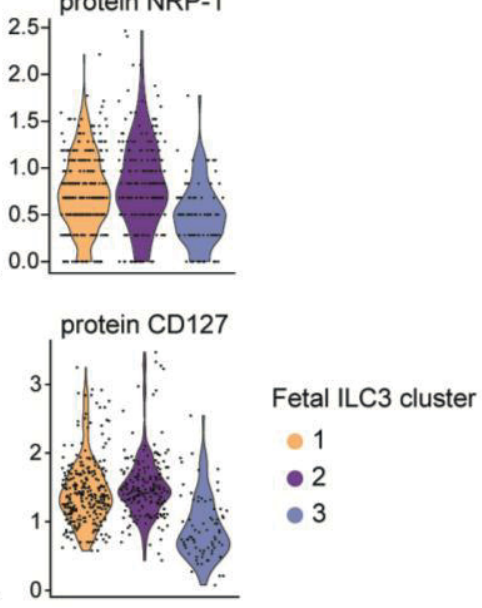

Figure 14. Transcriptomic profiling on single-cell level discloses heterogeneity within the embryonic ILC3 supercluster. (A, B) Violin plots of expressed transcripts or proteins within ILC3 subclusters. (C) Co-expression plots of Tbx21 and Ncr1 within ILC3 subclusters. (D) Violin plots of II7r transcripts or CD127 protein within ILC3 subclusters.

\subsection{A subset of $\mathrm{ROR} \mathrm{t}^{+} \mathrm{T}^{-b e t^{+} \mathrm{CD} 4^{+}}{ }^{+}$ILC3 emerges at E14.5 during embryonic development}

To validate the scRNA-seq data by flow cytometry, bacterial artificial chromosome (BAC) transgenic T-bet-ZsGreen reporter mice were used to accurately detect T-bet expressing ILC by flow cytometry. Further, to monitor when T-bet ${ }^{+}$ILC3 emerge during embryonic development, a kinetic analysis of T-bet and RORyt expression by LinLD $^{-} C D 45^{+} \mathrm{CD} 122^{+}$and/or $\mathrm{CD} 127^{+}$cells derived from the developing SI or FL was performed. First, T-bet-ZsGreen reporter signal was validated by intracellular staining of T-bet, showing full co-expression of T-bet and ZsGreen fluorescent proteins (Figure 15 A). Both ROR $\gamma \mathrm{t}^{+}$ILC3 and T-bet ${ }^{+}$ILC1/NK were present in SI and liver of E14.5 mice (Figure 15 B and C) where T-bet ${ }^{+}$subsets mainly comprised of ILC1 since Eomes was not detected (Figure 15 D, F, G). Within ROR $\gamma \mathrm{t}^{+}$ILC3, classical CD4 ${ }^{+}$and 
CD4- T-bet- LTi cells could be observed, as described previously (Eberl et al., 2004; Mebius et al., 2001). Besides classical LTi cells, around $25 \%$ of ROR $\gamma \mathrm{t}^{+}$ILC3 co-expressed T-bet and a fraction of these cells co-expressed CD4 (Figure 15 B and C). Similar subsets of ILC3 were dissected according to T-bet and CD4 expression were also observed in the E14.5 mesenteric LN ( $\mathrm{mLN}$ ) anlage (Figure 15 E). Further characterization of intestinal (Figure 15 F) and liver (Figure 15 G) ILC3 subsets showed high surface expression of CD127, NRP-1 and CXCR5 in the CD4 ${ }^{+}$T-bet subset, with intermediate levels in $\mathrm{CD}^{+}{ }^{+}$T-bet $^{+}$and lowest expression in CD4-T-bet ${ }^{+}$ cells. All ILC3 subsets expressed high levels of $\alpha_{4} \beta_{7}$; conversely, CD90 was enriched in T-bet ${ }^{+}$ compared to T-bet- ILC3 at E14.5 (Figure 15 F and G).

A LinLDCD $45^{+}$CD $122^{+}$and/or CD127+ B

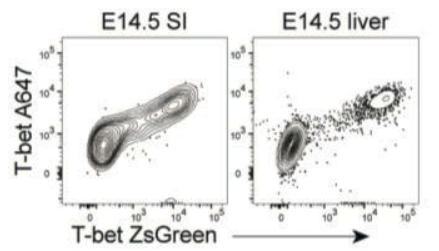

C

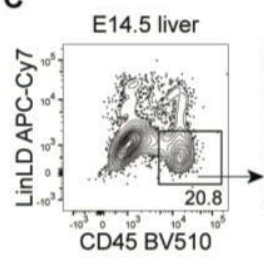

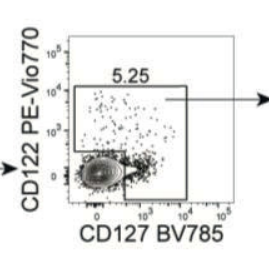

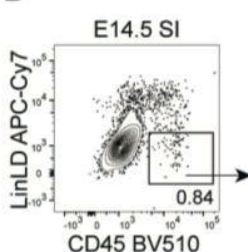

CD45 BV510

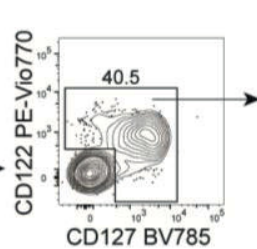

CD127 BV785

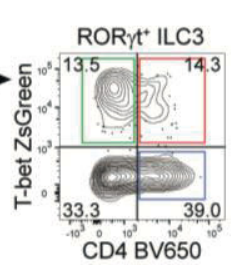

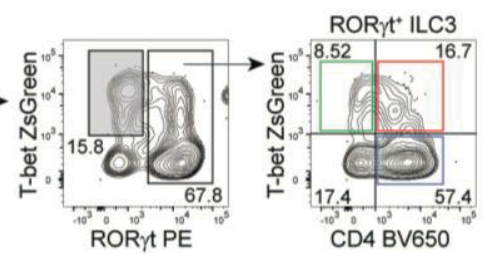

D LinLD CD $45^{+}$CD $122^{+}$and/or CD $127^{+}$

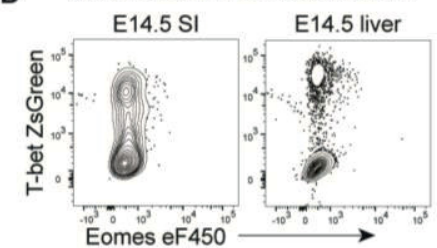

E

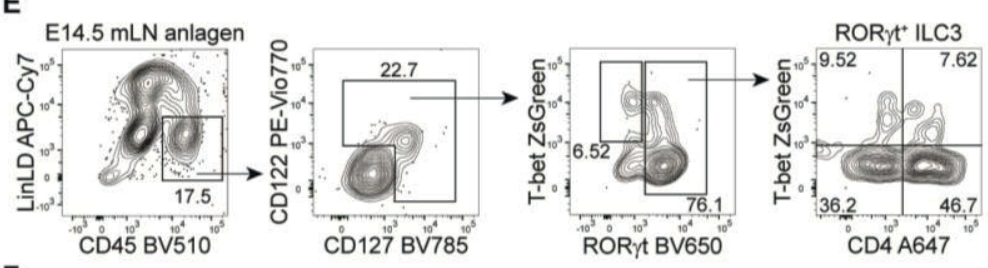

F

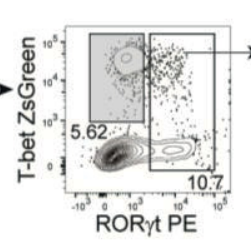

- CD4. T-bet ZsG' ILC3

- $\mathrm{CD}^{*}{ }^{*}$ T-bet ZsG ${ }^{*} \mathrm{ILC3}$

- CD4 ${ }^{*}$ T-bet ZsG-ILC3

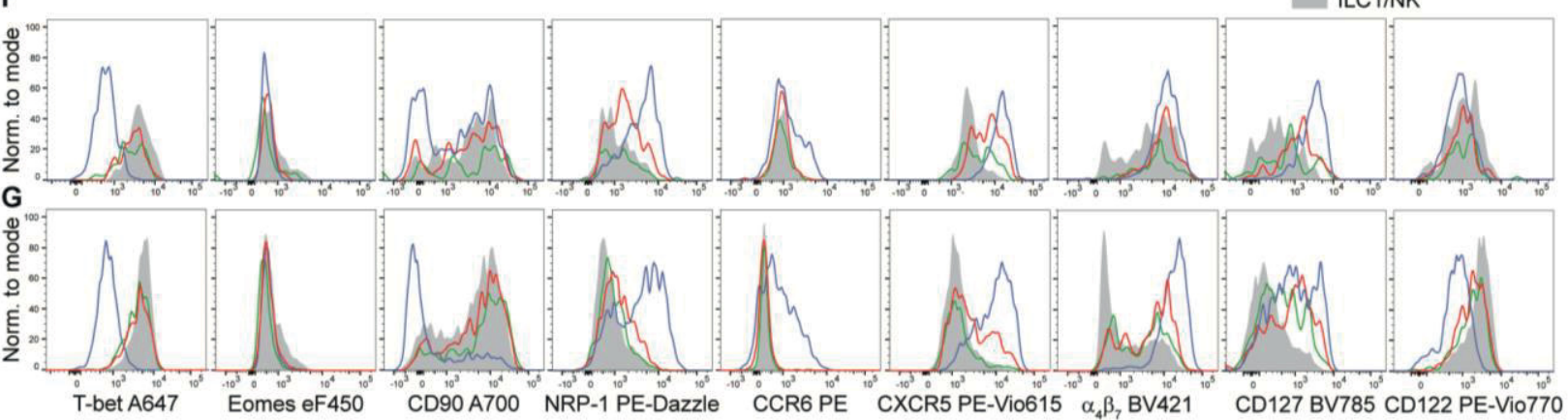

Figure 15. A subset of ROR $\mathbf{t}^{+} \mathbf{T}^{-}$-bet ${ }^{+} \mathrm{CD}^{+}{ }^{+}$ILC3 emerges during embryonic development. Flow cytometric analysis of E14.5 T-bet ZsGreen reporter mice. (A) Representative verification of T-bet protein and ZsGreen reporter signal in E14.5 small intestine (SI) or liver. Representative gating of E14.5 SI (B) or liver (C) for identification of T-bet ${ }^{+}$ROR $\gamma t^{-}$ILC1 (grey shaded gate) and ROR $\gamma \mathrm{t}^{+}$ILC3. (D) Representative plots of Eomes expression in E14.5 SI and liver. (E) Representative gating of E14.5 mLN anlagen (pooled sample) for identification of T-bet ${ }^{+} \mathrm{ROR} \gamma \mathrm{t}^{-}$ILC1 and ROR $\gamma \mathrm{t}^{+}$ILC3 subsets. Representative histograms of marker expression by ILC1/NK or ILC3 subsets based on expression of CD4 and T-bet ZsGreen from E14.5 SI (F) or liver (G). Data are representative of 2-3 independent experiments.

We next comparatively analyzed the kinetic of ILC subsets in SI and FL to understand whether frequencies of these populations varied in time pre- and postnatally. While the percentage of 
LinLD-CD45 ${ }^{+} \mathrm{CD} 122^{+}$and/or CD $127^{+}$cells was generally lower in FL than in SI (Figure 15 B and C), ILC3 represented the majority in SI and ILC1 were present at lower frequencies within group 1/3 ILCs at all time points (Figure $16 \mathbf{A}$ ). Although frequencies of small intestinal ILC1 and ILC3 converged at E18.5, ILC3 established as the major ILC population over time. In FL, comparable frequencies of ILC1 and ILC3 could be observed in E14.5 and percentages of ILC3 decreased successively with concomitant increase in ILC1 (Figure 16 A). The percentage of CD4-T-bet ${ }^{+}$ ILC3 increased gradually in the intestine, while CD4 ${ }^{+}$T-bet ${ }^{-}$and CD4 ${ }^{+} \mathrm{T}$-bet ${ }^{+}$ILC3 decreased from E14.5 to 4 weeks of age (Figure 16 B and C). In line with previous findings (Sawa et al., 2010), NKp46 was not detected on the surface of ILC3 during embryonic development and appeared only in newborn mice, regardless of T-bet expression (Figure 16 B). Expression of CCR6 was selectively observed by a minor fraction of intestinal CD4+T-bet ILC3 cells by E14.5 (Figure 15 F), while its expression increased until E18.5, when CCR6 ${ }^{+}$CD4- ILC3 first appeared (Figure 16 D). The frequency of $\mathrm{CCR} 6^{-} \mathrm{CD} 4^{+} \mathrm{ILC3}$ reduced progressively until virtually absent postnatally where $\mathrm{CCR} 6^{+} \mathrm{CD} 4^{+/-}$became the predominant ILC3 populations (Figure $16 \mathrm{D}$ ).

A
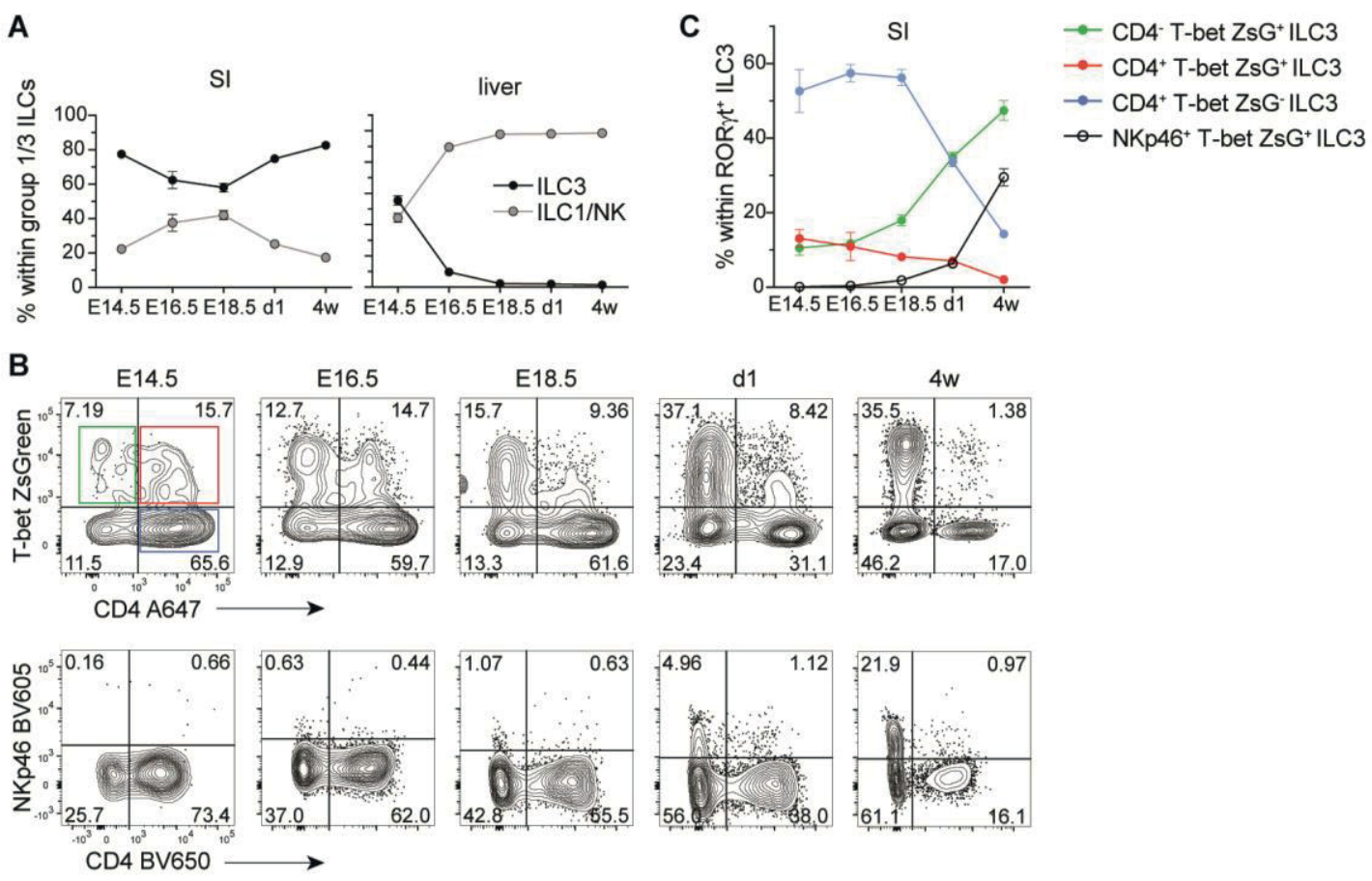

D
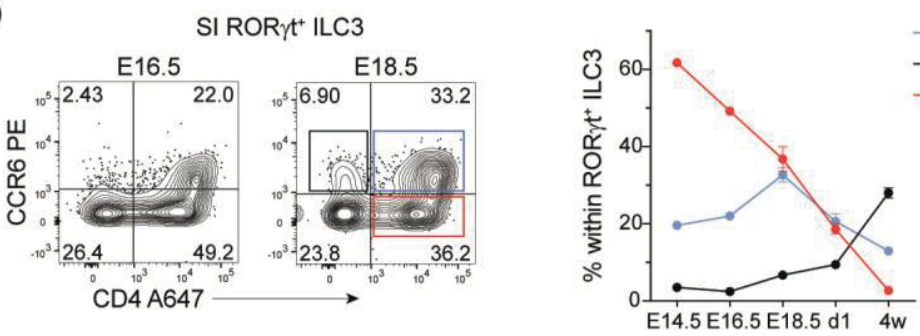

$\rightarrow \mathrm{CCR}^{+} \mathrm{CD}^{+}$

$\rightarrow$ CCR6-CD4 $4^{+}$

Figure 16. Kinetics of ILC3 subsets during ontogeny reveal distinct waves of differentiation. (A) Quantification of ILC1/NK or ILC3 frequencies within T-bet ${ }^{+}$and/or ROR $\gamma \mathrm{t}^{+}$ group 1/3 ILCs over time in ontogeny in SI and liver. (B) Representative plots of T-bet ZsGreen or NKp46 and CD4 expression by ROR $\gamma \mathrm{t}^{+}$ILC3 isolated from SI at different indicated embryonic and postnatal time-points. (C) Quantification of frequency of designated ROR $\gamma \mathrm{t}^{+}$ILC3 subsets over time in ontogeny. (D) Representative graphs of E16.5 and E18.5 and quantification over time of CCR6 and/or CD4 expression within ROR $\gamma \mathrm{t}^{+}$ILC3 isolated from SI. Graphs depict data as mean $\pm \mathrm{SEM}, \mathrm{n}=$ 3-12. Data are representative of 2-3 independent experiments. 
These data depict that a consistent proportion of CD4+ Tbet $^{+}$intestinal ILC3 was present at E14.5 in contrast to 4-week-old mice. To test whether embryonic CD4 ${ }^{+}$Tbet $^{+}$ILC3 might undergo lineage reprogramming already during fetal life, genetically fate-labelled mouse models that express cell-heritable fluorescent dyes in a cell type-specific manner were analyzed. These mice enable help to define relationships between cell types and provide information on lineage origin.

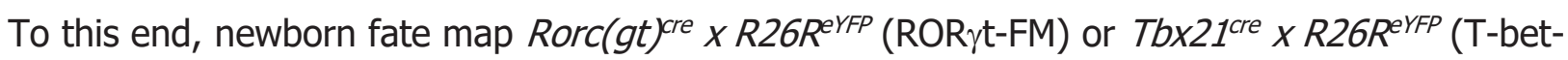
FM) reporter mice were analyzed as the frequency of $\mathrm{CD}^{+}{ }^{+}$Tbet $^{+}$ILC3 was already strongly diminished at birth. By analyzing ROR $\gamma \mathrm{t}-\mathrm{FM}$ mice indeed, a population of ILC3 was found that had switched off RORyt expression converting into ex ILC3 as it has also been previously described in adult intestine under the influence of IL-12 (Bernink et al., 2013; Klose et al., 2013; Vonarbourg et al., 2010). Specifically, a population of NK1.1+ROR $\gamma \mathrm{t}-\mathrm{FM}^{+}$cells was found within T-bet ${ }^{+}$ROR $\gamma t^{-}$ILC1 in both intestine and liver, showing that ex ILC3 are not only generated postnatally, but are already present at birth (Figure 17 A and B). Reciprocally, a population of T-bet-FM+ cells among ROR $\gamma \mathrm{t}^{+} \mathrm{CD}^{+}{ }^{+}$ILC3 was detected in the intestine of newborn T-bet-FM reporter mice, suggesting a history of T-bet expression among LTi cells (Figure $17 \mathrm{C}$ ).

A $\operatorname{Rorc}(g t)^{\text {cro }} \times R 26 R^{\text {oYFP }}, \mathrm{d} 1 \mathrm{SI}$
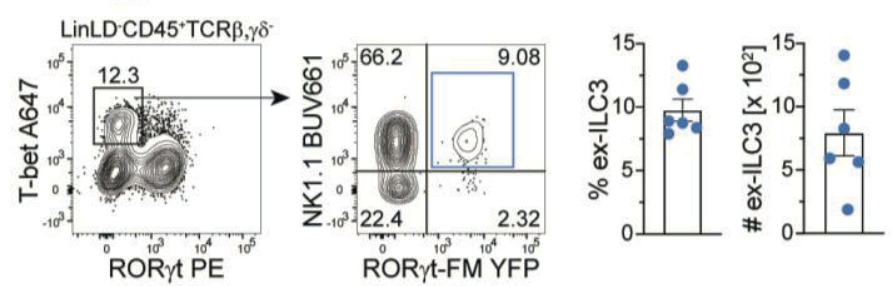

B

Rorc $(g t)^{\text {cre }} \times R^{2} 26 R^{\text {YYFP }}, \mathrm{d} 1$ liver
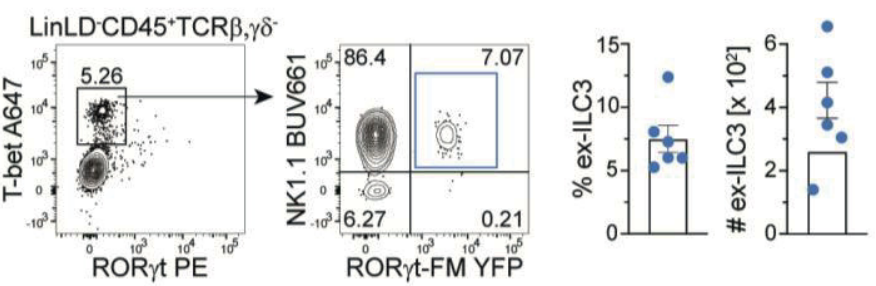

C ${ }^{b} \times 21^{\text {cro }} \times R 26 R^{\text {OYFP }}, \mathrm{d} 1 \mathrm{SI}$

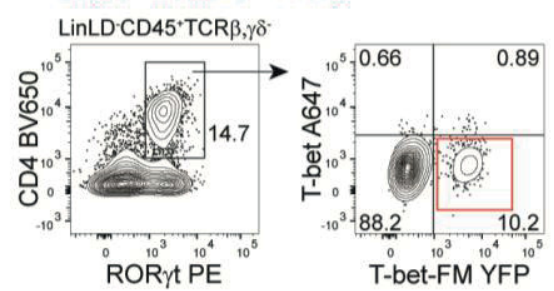

Figure 17. ILC3 plasticity starts prenatally. Representative gating on T-bet ${ }^{+}$ROR $\gamma$ t-protein ${ }^{-}$ ILC1 and identification of NK1.1 $1^{+} \mathrm{ROR} \gamma \mathrm{t}$-fate map $(\mathrm{FM})^{+}$ex-ILC3 in one day old Rorc(gt)cre/wt $x$ R26 YYFP newborn mice in SI (A) and liver (B). (C) Representative SI gating on ROR $\gamma t$ protein ${ }^{+} \mathrm{CD} 4^{+} \mathrm{LTi}$ cells and identification of $\mathrm{T}$ bet-protein-T-bet-FM+ ${ }^{+}$ILC3 in one day old Tbx21cre/wt $X \quad R 26^{\text {EYFP }}$ newborn mice. Quantification of frequencies and absolute numbers as mean \pm SEM. Data are representative of 2-3 independent experiments $(n=3-6)$ per time-point.

Altogether, T-bet was expressed by subsets of intestinal ILC3 during embryonic development; explicitly a population of CD4 ${ }^{+}$Tbet $^{+}$ILC3 was identified, which gradually decreased during development suggesting a possible conversion of ILC3 and ILC1 fates, highlighting a previously unappreciated prenatal ILC plasticity. 


\subsection{T-bet deficiency rescues formation of peripheral LN in ROR $\gamma t$-deficient mice}

Following up on the finding of T-bet expression in fetal ILC3, the effect of its expression on embryonic ILC3 functions, namely LN formation, was investigated next. To this point, $T b \times 21^{-1} x$ $\operatorname{Rorc}(g t)^{G F P / w t}$ mice were analyzed with $\operatorname{Rorc}(g t)^{G F P / w t}$ reporter mice as controls, demonstrating that $\mathrm{LN}$ generation in adult $\mathrm{Tb} \times 21^{--} \times \operatorname{Rorc}(g t)^{G F P / \omega t}$ mice was largely normal (Figure $18 \mathrm{~A}-\mathrm{C}$ ) (Rankin et al., 2013).
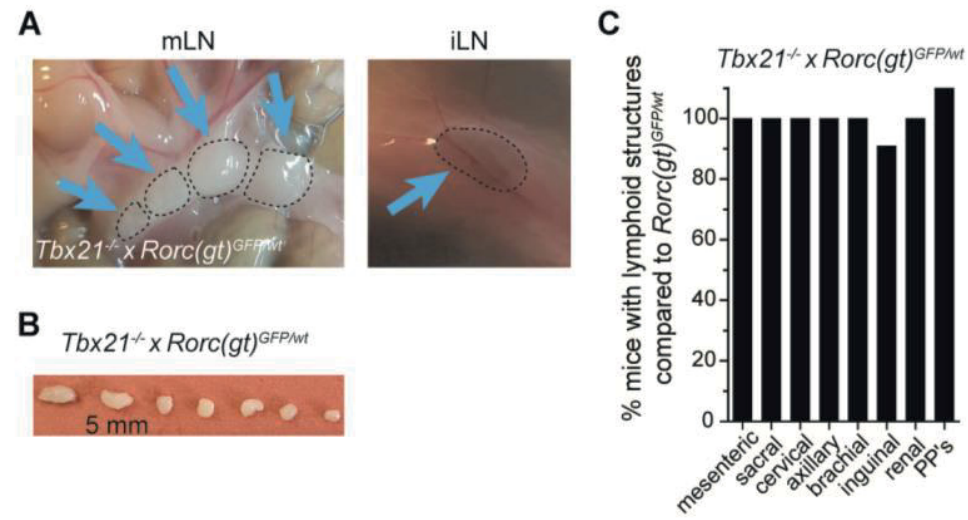

Figure 18. T-bet knockout mice have normal development of LN. (A) In vivo photos of mesenteric or inguinal lymph nodes (mLN, iLN) of adult $T b \times 21^{--} x$ $\operatorname{Rorc}(g t)^{G F P / G F P}$ mice or after isolation (B). (C) Quantification $(\mathrm{n}=12$ ) of frequencies of mice with lymphoid structures compared to control mouse strain in adult animals. PP's, Peyer's patches.

It is well established that T-bet and ROR $\gamma$ t are co-expressed within adult ILC3 where they crossregulate each other (Klose et al., 2013; Rankin et al., 2013; Zhong et al., 2016). To examine the influence of both TFs for embryonic ILC3 development, $\operatorname{Rorc}(g t)^{G F P / G F P} \times \operatorname{Tb} \times 21^{-}$double knockout (DKO) mice were generated. While RORyt deficiency in $\operatorname{Rorc}(g t)^{G F P / w t}$ mice led to dramatic impairment of peripheral LN generation as previously reported (Eberl et al., 2004; Sun et al., 2000), analysis of $\operatorname{Rorc}(g t)^{G F P / G F P} \times T$ Tb $\times 21^{1-}$ mice, lacking both T-bet and ROR $\gamma$, resulted in complete rescue of sacral, cervical, axillary and mesenteric LN, whereas emergence of brachial, inguinal and renal LN occurred in around 30-60\% and PP (Figure 19 A-C).
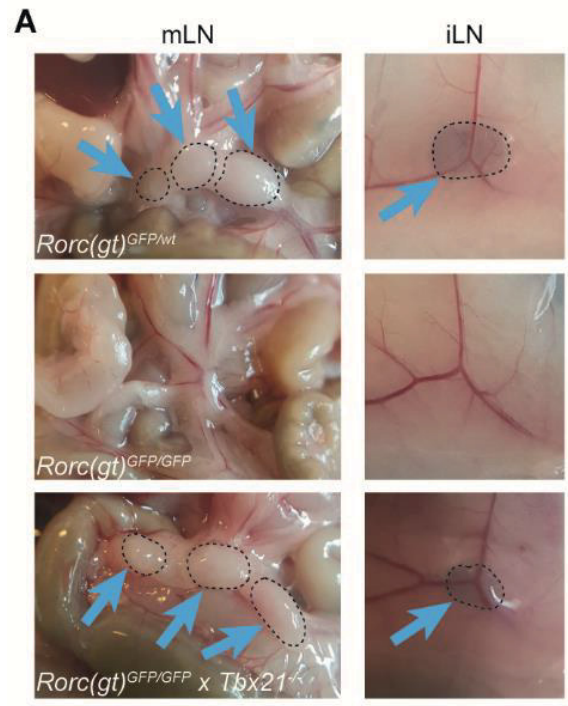

B

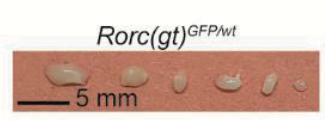

$\operatorname{Rorc}(g t)^{\text {GFP/GFP }} \times \operatorname{Tb} \times 21 \%$

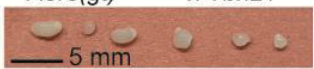

C

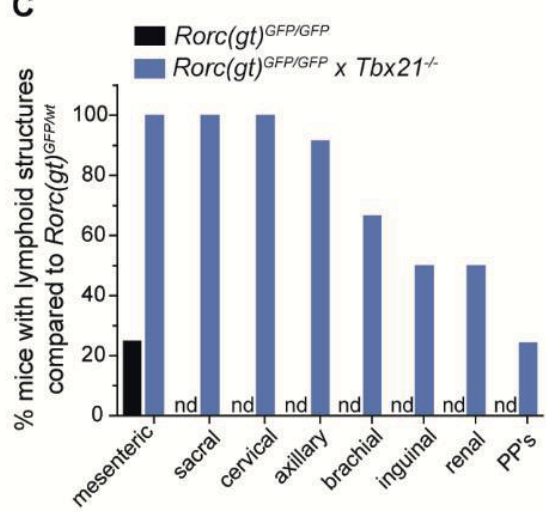

Figure 19. T-bet deficiency rescues $L N$ formation in ROR $\gamma$ t-deficient mice. (A) In vivo photos of mesenteric or inguinal lymph nodes ( $\mathrm{mLN}$, iLN) of adult animals in indicated mouse strains. (B) Photos of isolated $\mathrm{mLN}$ in indicated mouse strains. (C) Quantification ( $\mathrm{n}=12)$ of frequencies of mice with lymphoid structures compared to control mouse strain $\operatorname{Rorc}(g t)^{G F P / w t}$ in adult animals. PP's, Peyer's patches; nd, not detected. 
$\mathrm{LN}$ architecture and functions in $\mathrm{mLN}$ from $\operatorname{Rorc}(g t)^{G F P / G F P} \times \operatorname{Tb} \times 21^{\%}$ mice were normal, as evaluated by the presence of germinal centres (GCs) that were marked by PNA-binding B cell clusters and surrounded by $\operatorname{IgD}^{+}$naive B cells. GCs exhibited a discrete segregation of dark and light zone, where conventional $\mathrm{CD}^{+} 5^{+}$follicular dendritic cells were present (Figure 20 A). Moreover, flow cytometric analysis of $\mathrm{mLN}$ from $\operatorname{Rorc}(g t)^{G F P / G F P} \times \operatorname{Tb} \times 21^{-/-}$mice identified equal numbers of T follicular helper (Tfh) cells characterized by $\mathrm{CXCR} 5^{+} \mathrm{PD} 1^{+}$as well as ICOS and Bcl-6 expression in addition to $\mathrm{IgD}^{-/ 10} \mathrm{CD} 38^{-} \mathrm{Fas}^{+} \mathrm{GL7} 7^{+} \mathrm{GC}$ B cells when compared to control mice, revealing functional competence of these $\mathrm{mLN}$ (Figure $20 \mathrm{~B}$ ).

\section{A}
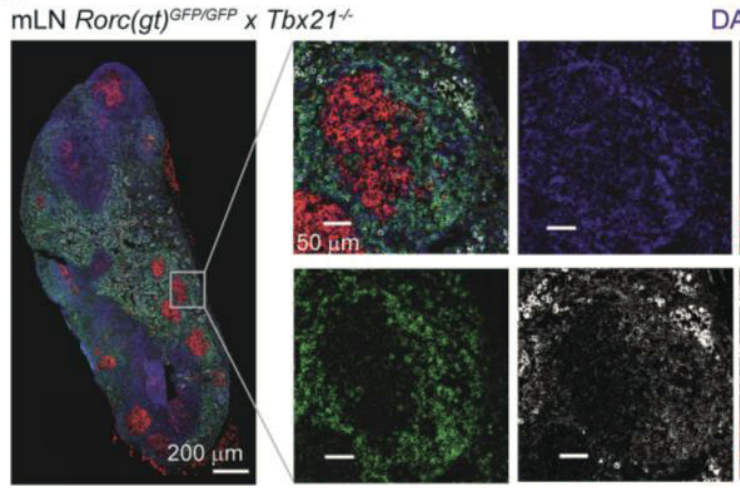

DAPI PNA IgD CD35
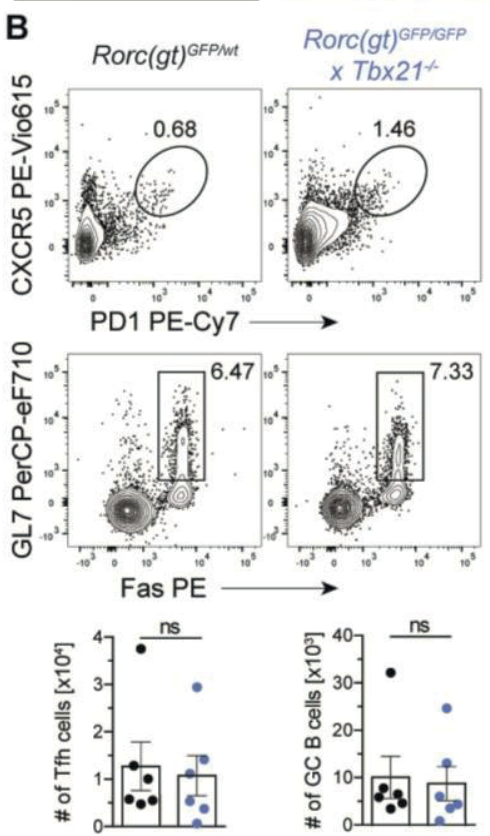
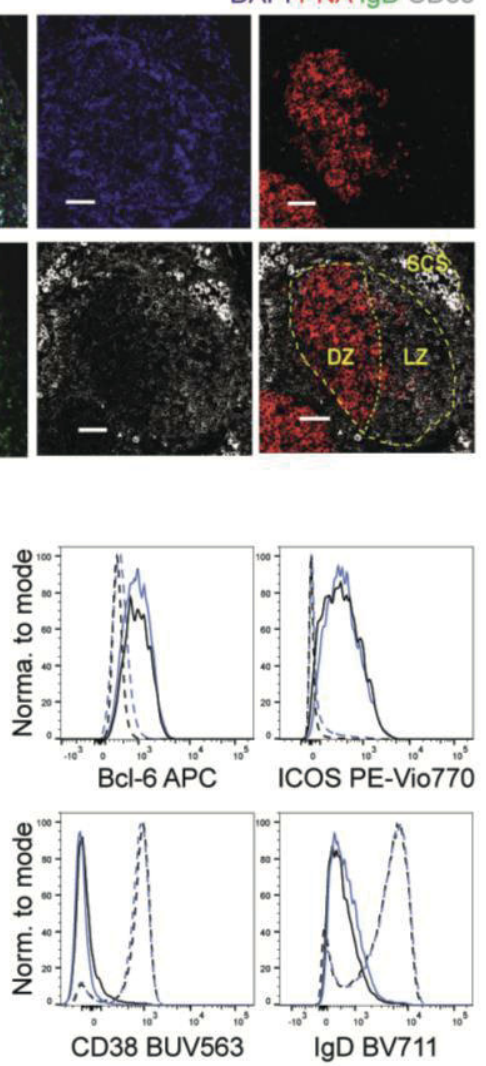

- Rorc(gt) GFPMt $^{\text {GPM }}$

- Rorc $(g t)^{\text {GFP/GFP }} \times \operatorname{Tb\times 21}$

::: ctrl. pop.
Figure 20. LN from ROR/t/T-bet-DKO mice have normal architecture and are functional. (A) Immunofluorescence of $\mathrm{mLN}$ in $\operatorname{Rorc}(g t)^{G F P / G F P} \times \mathrm{Tb} \times 21^{-1}$ mice. These data were acquired by $\mathrm{Dr}$. Carolin Ulbricht. DZ, dark zone; LZ, light zone; SCS; subcapsular sinus. (B) Representative flow cytometric plots of $\mathrm{T}$ follicular helper (Tfh) cells (upper row, gated as $\mathrm{LD}^{-} \mathrm{CD}_{4} 5^{+} \mathrm{CD}^{+}{ }^{+} \mathrm{CD} 4{ }^{+} \mathrm{CXCR}^{+}{ }^{+} \mathrm{PD} 1^{+}$) and germinal center (GC) B cells (lower row, gated as $\mathrm{LD}^{-} \mathrm{CD} 45^{+} \mathrm{CD}^{-}$ $\mathrm{B} 220^{+} \mathrm{CD} 19^{+} \mathrm{GL}^{+} \mathrm{Fas}^{+}$). Histograms show expression of Bcl-6 and ICOS in Tfh cells, control population consists of non-Tfh $\mathrm{CD}^{+}$ $T$ cells; and CD38 as well as IgD expression in GC B cells, control population consists of non-GC B cells. Black line represents $\operatorname{Rorc}(g t)^{G F P} / w_{t}$ mice, blue line represents $\operatorname{Rorc}(g t)^{G F P / G F P} \quad x \quad T b \times 21^{\%} \quad$ mice. Quantification of Tfh and GC B cells in the different mouse strains. Frequencies are shown as mean \pm SEM, data are representative of 2 independent experiments $(n=3)$ per time-point. MannWhitney U test; ns, not significant.

In order to exclude a possible role for T or B cells in shaping the observed LN phenotype,

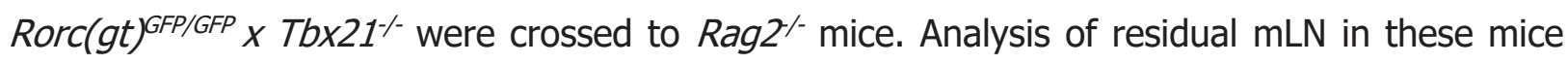
revealed analogously rescued development in $\operatorname{Rag} 2^{-1-} \times \operatorname{Rorc}(g t)^{G F P / G F P} \times \operatorname{Tb} \times 21^{1--}$ mice as opposed to $\operatorname{Rag}^{-1-} x \operatorname{Rorc}(g t)^{G F P / G F P}$ strain (Figure 21 A and B). 
A
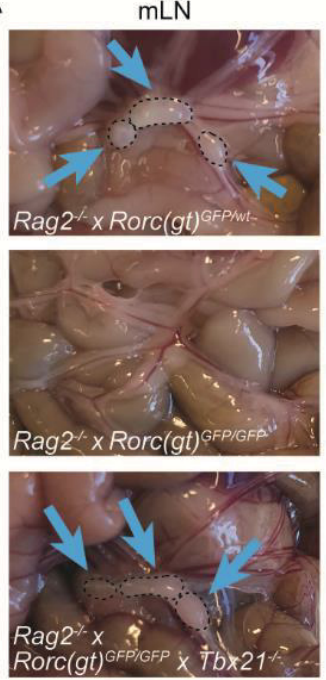

B

- Rag2 $2^{-1}$ Rorc $(g t)^{\text {GFP/GFP }}$ Rag 2 × Rorc $(g t)^{\text {GFP/GFP }} \times$ Tbx $21 \%$

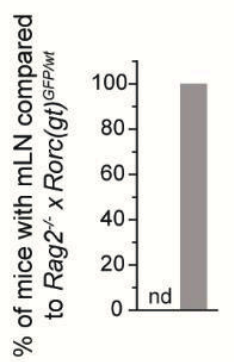

Figure 21. Re-appearance of $L N$ is independent of RAG. (A) Photos of mesenteric or inguinal lymph nodes ( $\mathrm{mLN}$, iLN) of adult animals in indicated mouse strains. (B) Quantification ( $\mathrm{n}=$ 12) of frequencies of mice with lymphoid structures compared to control mouse strain in adult animals. $\mathrm{Nd}$, not detected.

These results indicate that in the presence of ROR $\gamma$ t, T-bet does not affect embryonic LTi cell functions; however, in the absence of ROR $\gamma \mathrm{t}$, T-bet impedes LN formation independently of T and $B$ cells as evidenced by re-appearance of LN in RORyt and T-bet double knockout mice.

\subsection{Block of ILC1 and ILC3 differentiation in $\operatorname{RorC}(g t)^{G F P / G F P} \times \operatorname{Tb} \times 21^{-/-}$mice favors the accumulation of ILCP}

To address whether LN formation in $\operatorname{Rorc}(g t)^{G F P / G F P} \times \operatorname{Tb} \times 21^{--}$mice was associated with the presence of an ILC population displaying LTi cell functions, LinLD CD45 ${ }^{+}$CD $122^{+}$and/or CD $127^{+}$ cells isolated from E18.5 SI of $\operatorname{Rorc}(g t)^{G F P / G F P} \times \operatorname{Tb} \times 21^{-/-}$(DKO), were compared to $\operatorname{Rorc}(g t)^{G F P / G F P}$ (RKO) or Rorc(gt) GFP/wt (reporter) mice by scRNA-seq. Collective analysis of equalized cell numbers of all three mouse strains revealed overlapping ILC clusters displaying analogous gene expression signatures in RKO and DKO mice as seen in reporter mice, though with clearly different distributions (Figure 22 A and B). As expected from ROR $\gamma$ t deficiency, ILC3 populations were drastically reduced in both RKO and DKO strains. This effect was paralleled by a conspicuous increase of ILC2 in RKO and DKO mice as well as of ILC1/NK in RKO mice, indicating a competitive cross-regulation between the different ILC lineages in the absence of either TF (Figure 22 A and B). Due to T-bet deficiency, DKO mice were defective of the ILC1 cluster, while few cells expressing Eomes could be still detected as NK cells can still generate in mice lacking T-bet (Figure 22 C) (Townsend et al., 2004). Investigating the progenitor populations, a remarkable overrepresentation of the ILCP cluster was observed in DKO mice, while frequencies of CLP remained equal (Figure 22 A and B). Gene signatures such as ILClineage markers Id2, Ets1, Rora, Ahr, CXCr6, Arg1, II7r and I/2rb, as well as of ILCP-associated genes Zbtb16, Tcf7 and Tox showed comparable expression in ILCP from all strains (Figure 22 D and E). In addition, $\alpha_{4} \beta_{7}$ and PD-1 was detected on the surface of DKO ILCP (Figure 22 F), 
as also seen in ILCP of reporter mice (Figure 12 B). Altogether, comparable analysis of all mouse strains by scRNA-seq exposed an accumulation of ILCP in DKO mice that was analyzed in more detail next to reveal potential association to LN re-appearance.

A
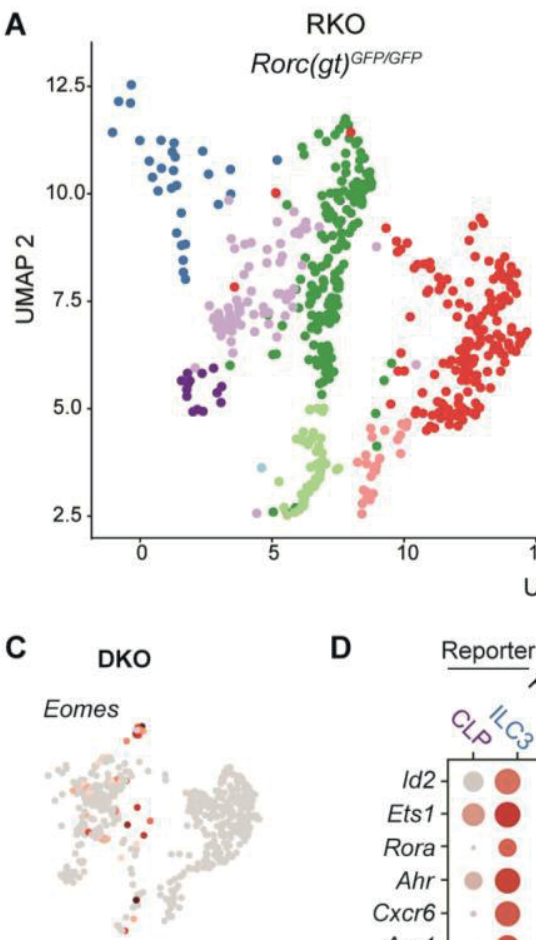

D

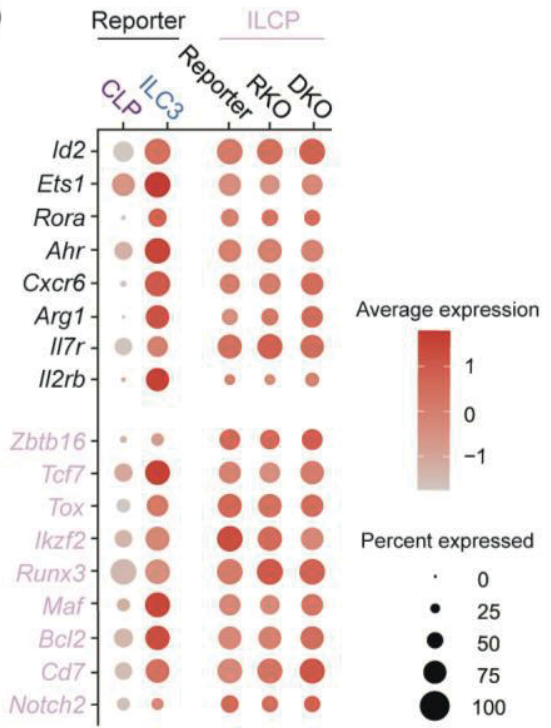

E

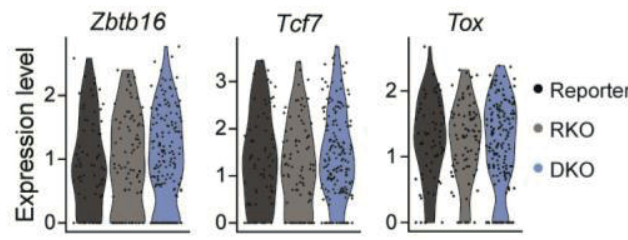

$\mathbf{F}$

DKO

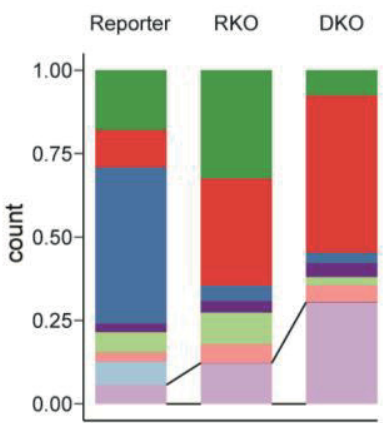

Figure 22. Single-cell RNA sequencing of fetal ILC populations reveal accumulation of ILCP in ROR $\gamma \mathbf{t} / \mathrm{T}-$ bet-DKO mice. Viable Lin(CD19, CD3, CD5, F4/80, FceRIa, Gr-1) ${ }^{-}$CD45 $5^{+}$cells expressing IL-7 receptor (CD127) and/or the IL-2 receptor subunit beta (CD122) isolated from the small intestine (SI) of E18.5 Rorc(gt)GFP/GFP(RKO) and $\operatorname{Rorc}(g t)^{G F P / G F P} \times \operatorname{Tb} \times 21^{-1}$ (DKO) embryos were sort-purified by flow cytometry, and a single-cell expression library was generated using 10x Genomics. (A) UMAP dimensional reduction projection identifies 8 distinct clusters. (B) Quantification of single cells mapping to correspondent clusters (legend see in A) in indicated mouse strains. (C) Selected gene expression within clusters and mouse strains. CLP and ILC3 from reporter mice compared to ILCPS of all strains. Color scale represents average expression, dot size visualizes fraction of cells within the cluster expressing the gene. (D) Expression of selected ILCP-associated genes in cells from ILCP cluster. (E) Expression of selected proteins on UMAP projection analyzed by CITE-Seq. (F) Selected protein expression on cells from DKO mice on UMAP plots.

\subsection{PLZF ${ }^{\text {hi }}$ ILCP from DKO mice are enriched in cells with LTi signatures}

Following up on the finding of increased ILCP in DKO mice, their transcriptional profile and differentiation commitment in DKO, RKO and reporter ILCP was further examined by evaluating the enrichment score of ILCP, ILC1, ILC2 or ILC3 gene modules, defined in the unperturbed subsets of reporter mice (Figure 23 A). As a result of ROR $\gamma$ t deficiency, RKO ILCP were enriched 


\section{Results}

for the ILC1 and ILC2, but not for the ILC3 module (Figure $23 \mathbf{A}$ ). In this regard, Tbx21 transcripts were more abundant in ILCP of RKO than in reporter mice (Figure 23 B), suggesting that in the absence of ROR $\gamma$ t counterbalance, expression of T-bet is enforced and skews ILCP maturation towards ILC1 lineage. On the contrary, the additional lack of T-bet in DKO mice caused accumulation of an ILCP population displaying higher enrichment score for ILC3, but not for ILC1 or ILC2 core modules (Figure 23 A). Interestingly, DKO ILCP with greater enrichment for ILC3 genes exhibited a lower enrichment score for the ILCP gene module (Figure 23 C). Examination of the top 100 genes defining the ILC3 core module in ILCP from DKO mice revealed no or only little expression of typical ILC3 genes, such as I/1r1, I/17re, Nrp1 or Ccr6, similar to their ILCP counterparts from reporter or RKO strains (Figure 23 D and E). In contrast, a fraction of DKO ILCP expressed higher transcripts for a defined set of ILC3 identifying genes as compared to reporter or RKO mice. Besides Gpr183, Batf3 and BCl2 (Figure 23 and F), DKO ILCP expressed higher levels of transcripts for genes associated with LTi cell phenotype and functions, namely $L t a, L t b, C X C r 5$ and Tnfsf11 (encoding RANKL) (Figure $23 \mathrm{G}$ ). Of note, while the absence of ROR $\gamma \mathrm{t}$ in both RKO and DKO mice clearly affected expression of ROR $\gamma$ t-driven signatures evidenced by the absence of ILC3 clusters (Figure 22 A and B), residual Rorc transcripts could still be observed within the ILCP from RKO and DKO strains (Figure 23 D), possibly indicating residual Rorc promoter activity. 

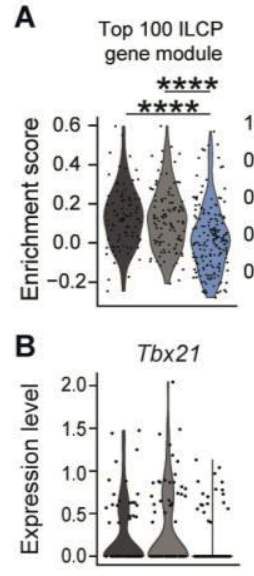

ILCP from

- Reporter

- RKO

- DKO

E

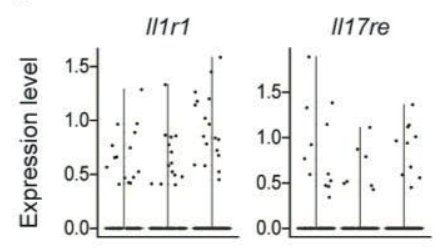

F
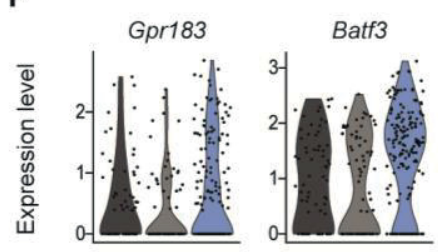

G
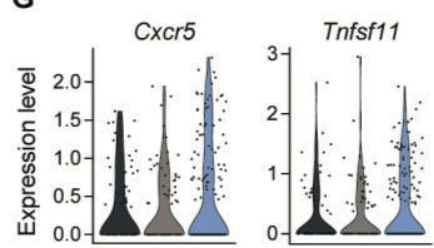

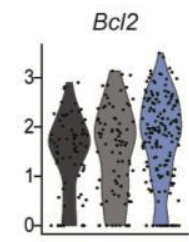

ILCP from

- Reporter

- RKO

- DKO
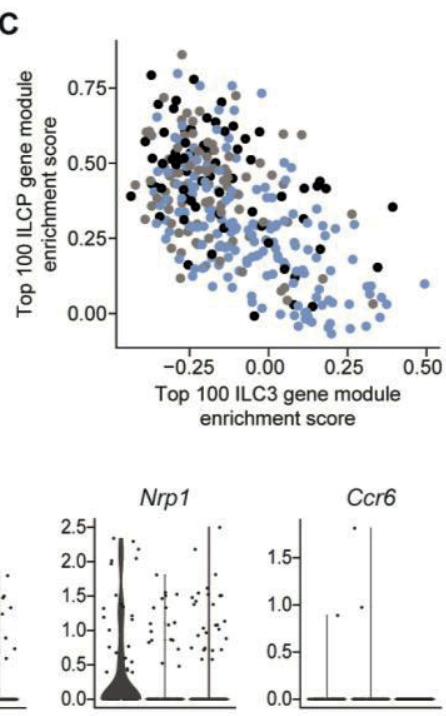

(20)
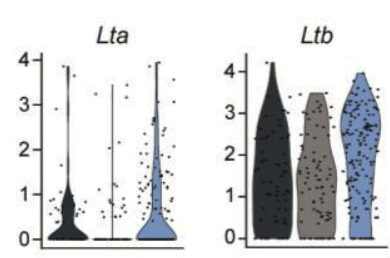

D
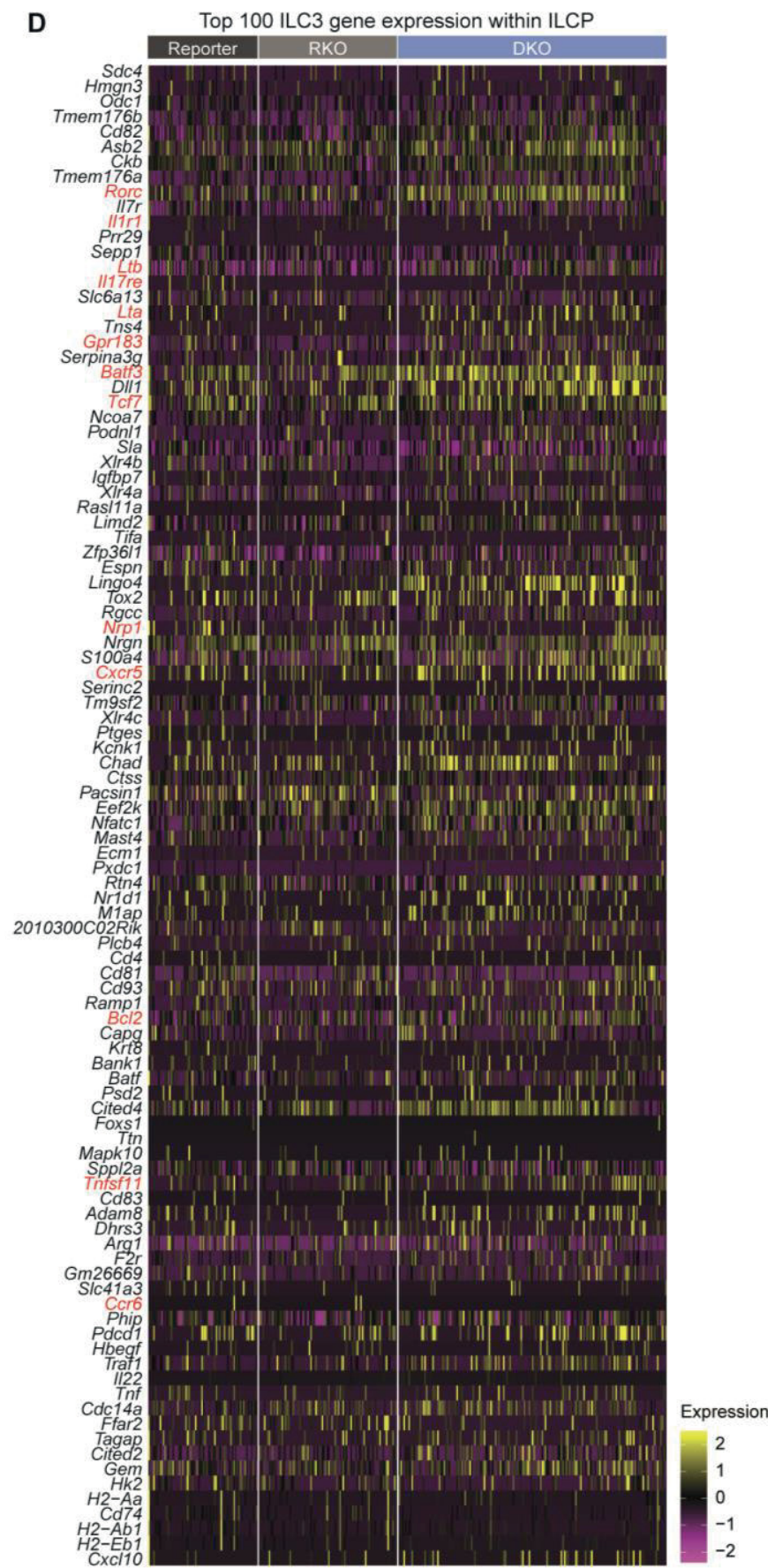

Top 100 ILC3 gene expression within ILCP

Figure 23. Gene expressions patterns in ILCP show enhanced LTi expression patterns in DKO mice. (A) Violin plots for enrichment score of gene modules of top 100 differentially expressed genes from ILCP or ILC1, ILC2, ILC3 cluster, defined in reporter mice, in single cells from ILCP cluster in indicated mouse strains. E18.5 scRNA-seq dataset, legend see in B. Statistical significance was calculated using Wilcoxon test with Bonferroni correction (B) Expression levels of Tbx21 within ILCP of all strains. (C) Co-enrichment plots of ILCP versus ILC3 gene modules within single cells of ILCP cluster in mouse strains. (D) Gene expression profiles of the top 100 genes differentially expressed in ILC3 of reporter mice within cells from ILCP cluster. (E-G) Violin plots depicting expression of selected transcripts in ILCP of the three mouse strains, legend see in B.

In order to validate the data generated by scRNA-seq, additional flow cytometric analysis of LinLD$^{-} C D 127^{+}$and/or CD122+ cells derived from E18.5 SI and mLN anlagen was performed. Among $\mathrm{CXCR6}^{+}$cells, a GFP ${ }^{+}$population was detected in DKO and to lower frequencies also in RKO mice indicating Rorc promoter activity and translation of the knock-in sequence encoding GFP in these mice. The observed GFP signal was in line with the observed Rorc transcripts by scRNA-seq (Figure 24 A). Furthermore, cells expressing LTi functional molecules such as the 
$\mathrm{LT} \alpha_{1} \beta_{2}$ heterotrimer, as well as CXCR5 and RANKL was present in Si and $\mathrm{mLN}$ at comparable frequencies to reporter mice while strongly reduced in RKO mice (Figure 24 B, C, E). Notably, in reporter mice these populations largely comprised of ROR $\gamma \mathrm{t}^{+} \mathrm{ILC} 3$. Although generally detected at low frequencies in FL, the frequency of CXCR5 ${ }^{+} R A N K L^{+}$ILCs was increased in DKO mice, suggesting that skewed ILCP differentiation towards acquisition of LTi signatures in DKO mice seems to occur already in the FL (Figure $24 \mathrm{D}$ ). In order to address whether $\mathrm{CXCR6}^{+}$cells in DKO mice corresponded to ILCP, expression of PLZF was assessed. Whereas CXCR6 ${ }^{+}$ILCs from reporter mice expressed low levels of PLZF, CXCR6+PLZFhi cells were selectively enriched in SI and $\mathrm{mLN}$ of DKO mice (Figure 24 F), where co-expression of PD-1 was seen likewise (Figure 24 G).

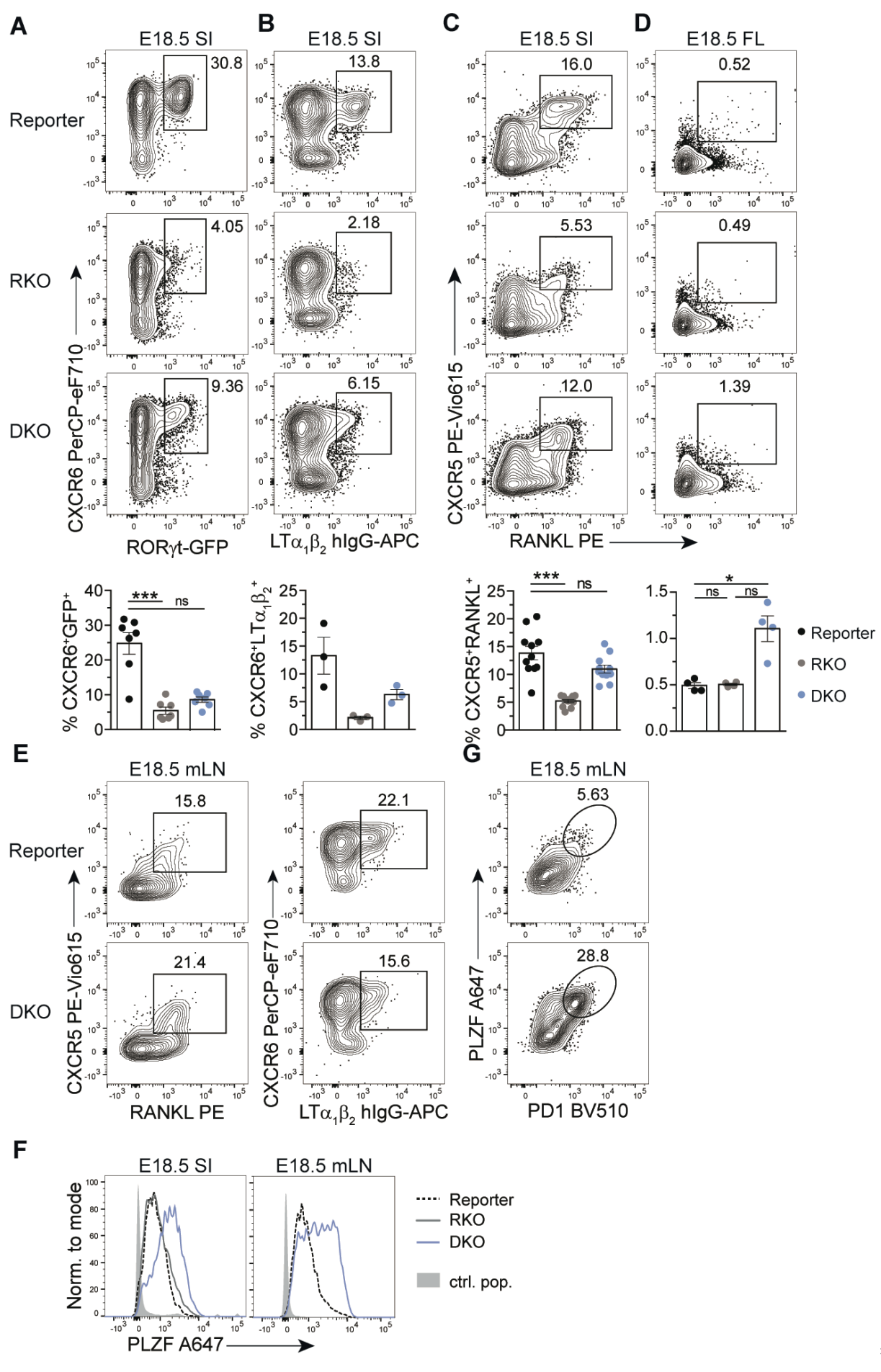


Figure 24. ILCP from DKO mice are enriched in cells with LTi signatures. (A) Representative flow cytometry from E18.5 SI of LinLD-CD45 ${ }^{+}$CD $127^{+}$and/or CD122 ${ }^{+}$cells. (A) Quantification CXCR6 ${ }^{+} \mathrm{GFP}^{+}$cells $(\mathrm{n}=7)$ and (B) of CXCR6 ${ }^{+} \mathrm{LT}_{1} \beta_{2}{ }^{+}$cells $(n=3)$ in the different mouse strains shown, see legend in $D$. (C) Quantification of CXCR5 $^{+}$RANKL $^{+}(n=10)$ in SI or fetal liver (FL) in (D). A-D, Kruskal-Wallis significance and Dunn's correction. (E) Representative flow cytometric plot of pooled E18.5 mLN anlagen gated on LinLD ${ }^{-} \mathrm{CD}_{4} 5^{+} \mathrm{CD} 127^{+}$and/or CD122 ${ }^{+}$cells. (F) Representative flow cytometric histogram of LinLD ${ }^{-} \mathrm{CD}_{4} 5^{+} \mathrm{CD} 127^{+}$and/or CD $122^{+} \mathrm{CXCR} 6^{+}$cells; control population (ctrl. pop.) defined as CD127 $\mathrm{CD} 122^{-}$of reporter mice. (G) Representative flow cytometric plot of pooled E18.5 mLN anlagen gated on LinLD-CD45+CD127+ and/or CD122+ cells.

To find out whether accumulation of DKO ILCP was due to enhanced proliferation, Ki67 expression was analyzed and revealed that transcripts as well as protein expression were comparable in ILCP from all three strains, thereby excluding this mechanism for increased ILCP in DKO mice (Figure 25 A-C).

A

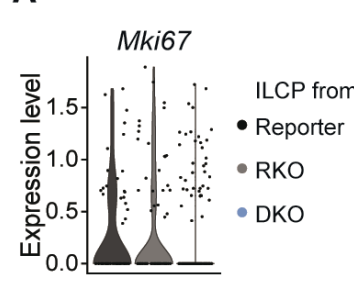

B

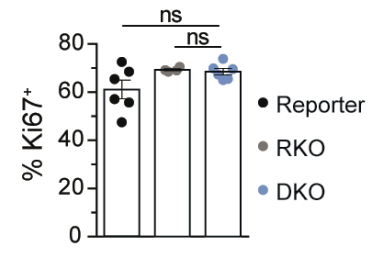

C

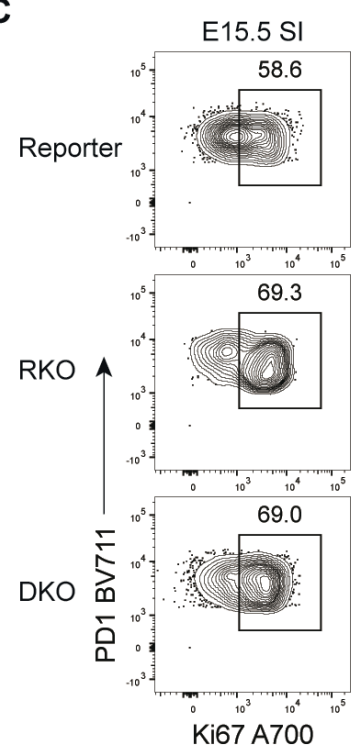

Figure 25. Equal Ki67 expression within ILCP of reporter, RKO and DKO mouse strains. (A) Violin plot for Mki67 transcripts among ILCP in all mouse strains, legend see in B. (B, C) Quantification and representative flow cytometry of Ki67 expression in LinLD$\mathrm{CD}^{2} 5^{+} \mathrm{CD} 127^{+}$ROR $\gamma$ tPD1 ${ }^{\text {hipLZF }}{ }^{\text {hi }}$ cells from E15.5 SI $(\mathrm{n}=$ 4-6). Quantification as mean \pm SEM with Kruskal-Wallis significance and Dunn's correction.

Thus, both scRNA-seq and flow cytometry analysis identified an ILCP population expressing CXCR6, PLZF, PD-1 and key LTi molecules that accumulated in FL, SI and mLN anlage of DKO mice. Overall, these results imply that lack of functional ROR $\gamma$ t prevents the development of fully mature ILC3; however, in the absence of T-bet, differentiation of ILCP with distinct LTi features is enabled.

To complement the in vivo data, in vitro differentiation assays culturing sorted FL-derived progenitors on stromal OP9 feeder cells were performed. After one week of culture in the presence of stem cell factor (SCF) and IL-7, all ILC lineages could be generated from progenitors obtained from reporter mice (Figure 26 A, B). As also seen ex vivo, ILC2 differentiation was increased in cultures from RKO and DKO mice, while generation of NK1.1+ cells (marking both ILC1 and NK cells) was additionally reduced in DKO as compared to RKO mice (Figure 26 A). Importantly, cultures from DKO, but not from RKO, progenitors enabled the generation of a population of RANKL ${ }^{+}$CXCR5 ${ }^{+}$cells, which co-expressed PLZF (Figure 26 B). 


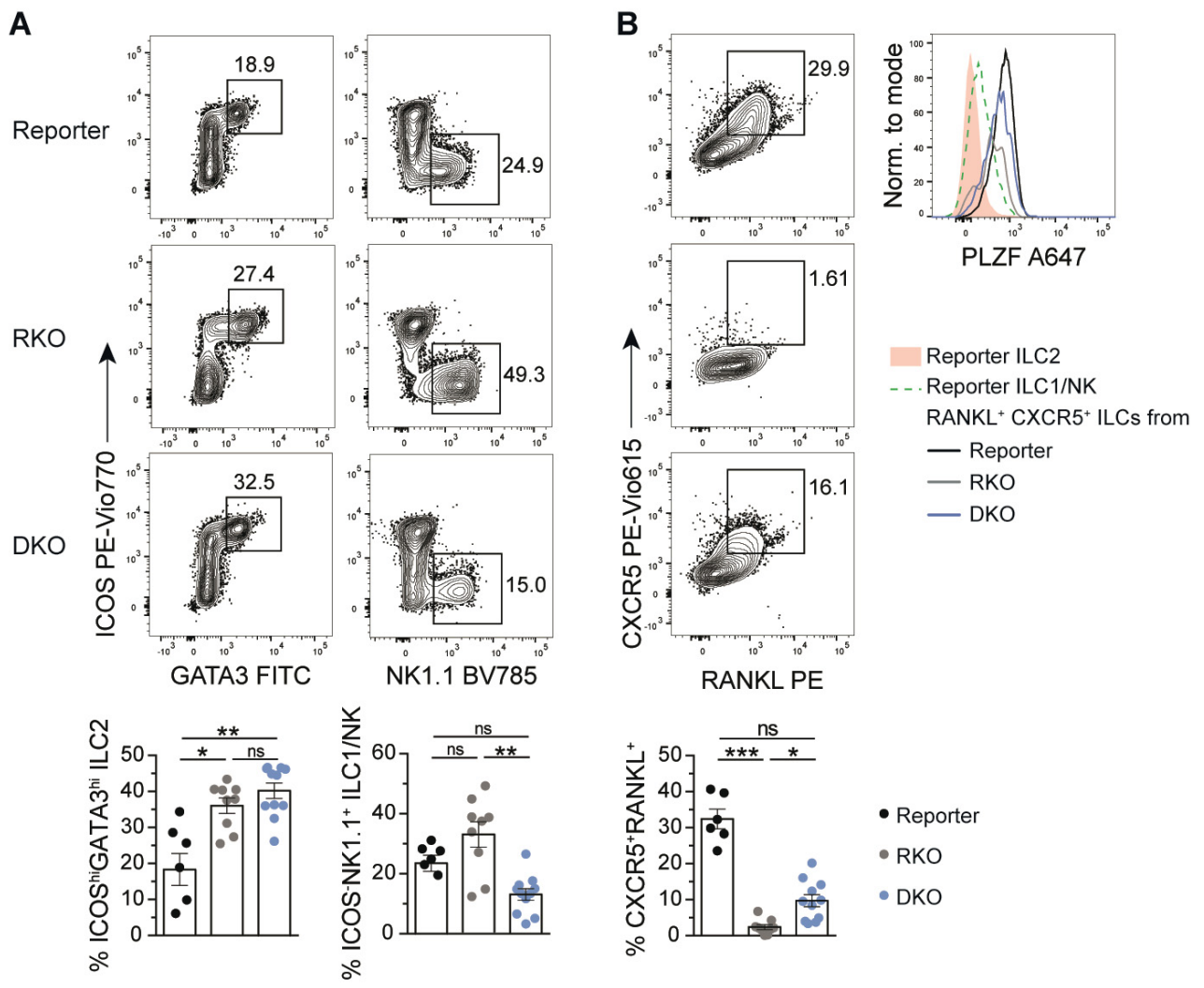

Figure 26. In vitro OP9 differentiation cultures. In vitro differentiation of E14.5 fetal liver-derived ILC progenitors on OP9 stromal cells after culture for 5-8 days in the presence of SCF and IL-7 and analysis by flow cytometry. (A) Representative flow cytometry plots and quantification of LinLDCD45 ${ }^{+}$ICOS $^{\text {hi GATA3 }}{ }^{\text {hi }}$ ILC2 and LinLDCD45 ${ }^{+}$ICOS$^{-N K 1.1^{+}}$group 1 ILCs cells in the different mouse strains, see legend in $B$. (B) Representative flow cytometry plots of $\mathrm{CXCR}^{+} \mathrm{RANKL}^{+}$cells among LinLDCD45 ${ }^{+}$GATA3 ${ }^{\text {hi-negNK1.1 }}{ }^{-}$in the different mouse strains. Quantification shown as mean \pm SEM, data are representative of 2-3 independent experiments $(n=3-5)$ per timepoint. Kruskal-Wallis significance and Dunn's correction.

\subsection{PLZFi ${ }^{\text {hXCR6 }}{ }^{+}$GFP $^{+}$cells persist in $\mathrm{mLN}$ and intestine of adult DKO mice}

Having identified an ILCP population with LTi features in embryos of DKO mice, 4-week-old mice were examined next to investigate whether such a population would persist into adulthood. $\mathrm{CXCR6}^{+} \mathrm{PLZF}^{+}$and $\mathrm{GFP}^{+}$cells were present in $\mathrm{mLN}$ and SI of adult DKO mice (Figure $27 \mathrm{~A}-\mathrm{C}$ ). Importantly, the GFP signal resolved GFPlo cells found in both RKO and DKO mice as well as GFPhi ILCs which were absent in SI of RKO but detected at similar frequencies in DKO and reporter mice (Figure $27 \mathrm{C}$ ). Conversely to RKO mice, DKO GFP+ ILCs partially expressed CD4, but not

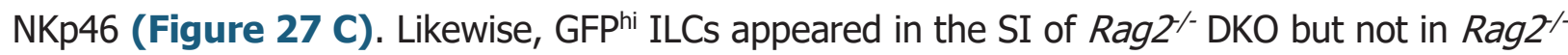
RKO mice (Figure 27 D). 

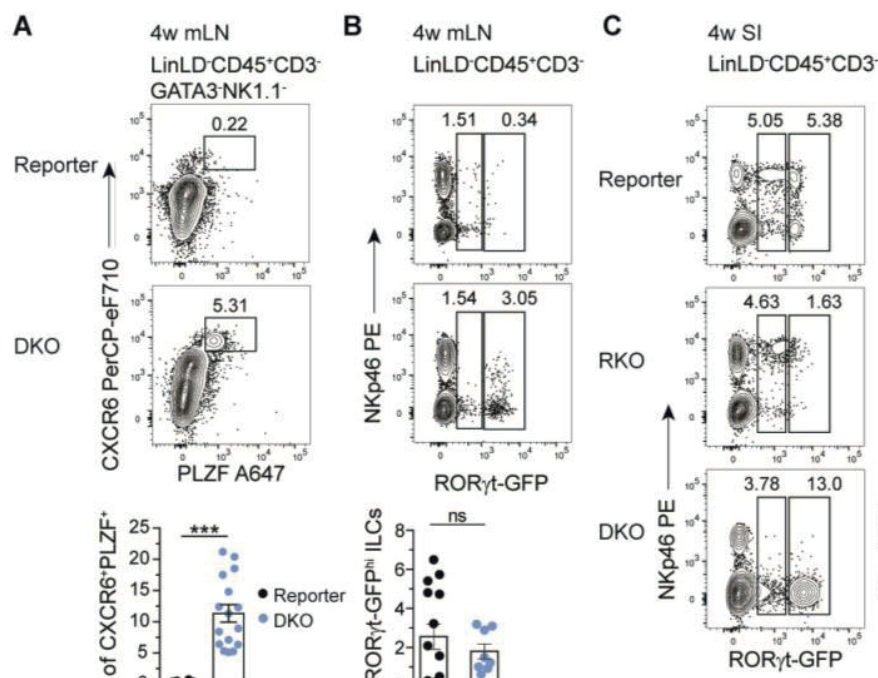

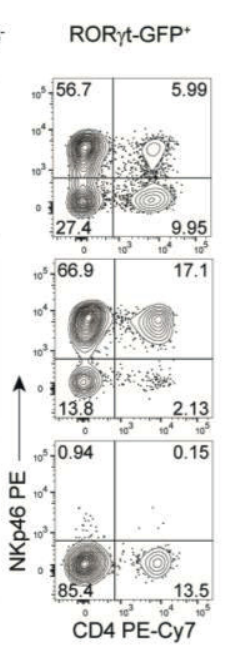

D
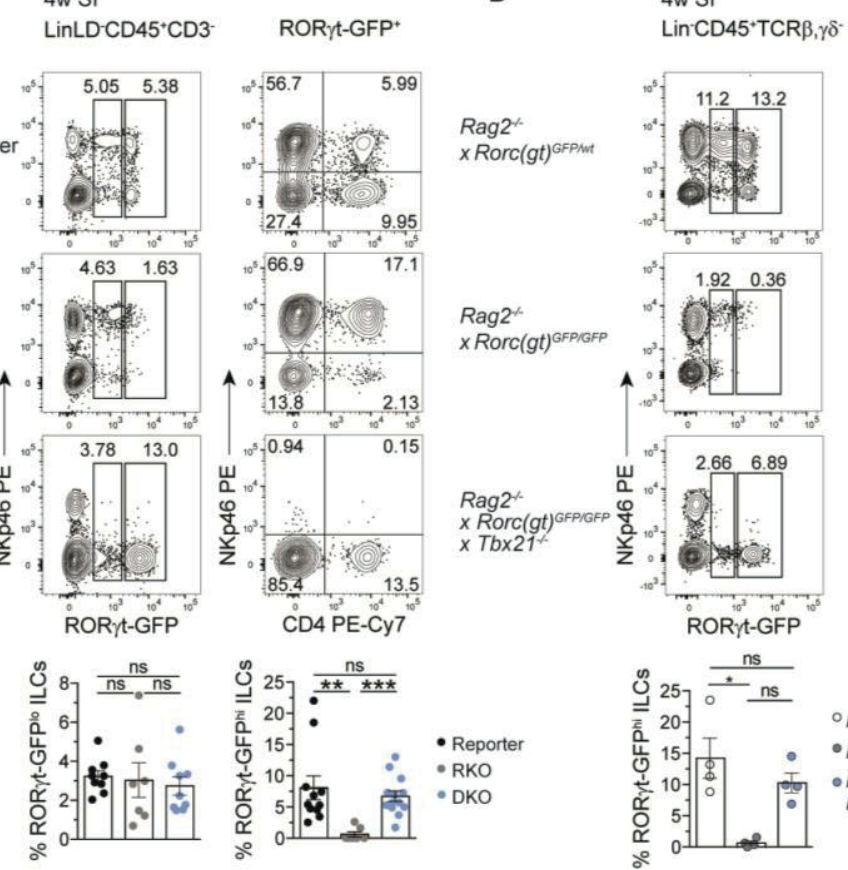

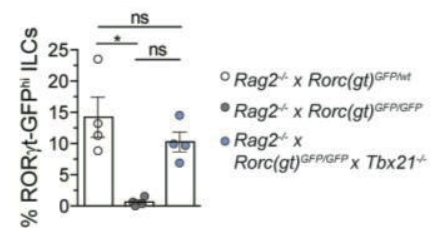

Figure 27. PLZFi CXCR6+ ${ }^{+}$ILCP persist in LN and intestine of adult DKO mice. (A, B) Flow cytometry representative plots of $\mathrm{mLN}$ from 4-week-old reporter and DKO mice. Quantification of frequencies from two to three independent experiments $(n=8-16)$, mean \pm SEM and Mann-Whitney-U testing. (C, D) Representative flow cytometry plots of SI from indicated mouse strains. Quantification of ROR $\gamma$ t-GFPlo and ROR $\gamma$ t-GFPhi populations. Data depicted as mean \pm SEM, Kruskal-Wallis testing with Dunn's multiple correction, $n=4-12$.

Bulk RNA-sequencing of $\mathrm{NKp} 46^{+}$and of $\mathrm{CD}^{+} \mathrm{GFP}^{+}$populations sorted from SI of adult reporter, RKO and DKO mice underlined the observations gained in the E18.5 single-cell approach. DKO $\mathrm{CD}^{+} \mathrm{GFP}^{+}$cells expressed abundant transcripts for Zbtb16, Tox and Tcf7, while lacking other ILC3-related genes including I/1r1 and Ccr6. Of note, CD4 ${ }^{+} \mathrm{GFP}^{+}$cells from DKO and reporter mice displayed comparable levels of transcripts for central LTi cell molecules, such as $L t a, L t b$, Tnfsf11 and CXCr5 (Figure $28 \mathrm{~A}$ ). Additionally, I/22, but not I/17a, transcripts were restored to similar amounts in DKO CD4+ GFP $^{+}$cells as in $\mathrm{NKp} 46+^{+}$ILC3 from reporter mice (Figure 28 A and B). These data could be confirmed at the protein level by intracellular staining of cytokines after ex vivo restimulation, showing that DKO $\mathrm{CD}^{+} \mathrm{GFP}^{+}$cells showcased IL-22, but not IL-17A protein, in line with the transcriptional data (Figure $28 \mathrm{C}$ ). Since IL-22 is crucial for induction of AMP it has an essential role in barrier homeostasis within the intestinal epithelium. To test whether partial restoration of IL-22 expression in DKO ILCs could rescue the expression of transcripts encoding for Reg $3 \gamma$ and Reg3 $\beta$ proteins in the intestinal epithelium, Reg $3 g$ and $R e g 3 b$ transcripts were measured in the SI epithelial cells from DKO, RKO and reporter mice. Notably, while RKO mice displayed dramatic reduction in Reg $3 g$ and Reg3b transcripts, DKO had comparable levels to reporter mice, indicating that partial IL-22 restoration observed in DKO ILCS is sufficient to restore important features of the intestinal barrier (Figure $28 \mathrm{D}$ ). 
A

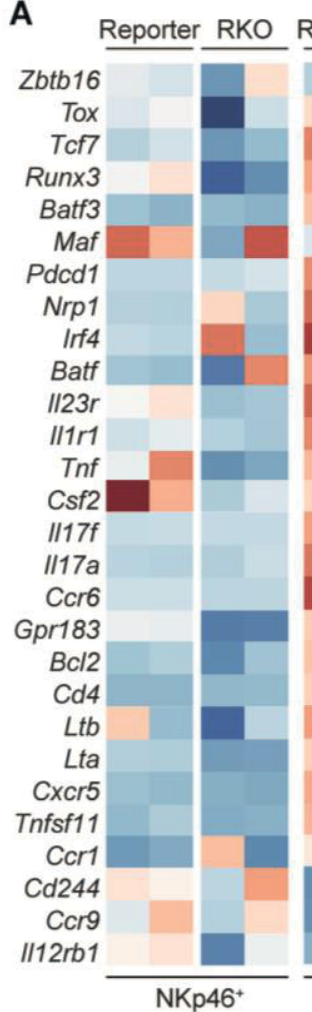

NKp46+
Reporter DKO

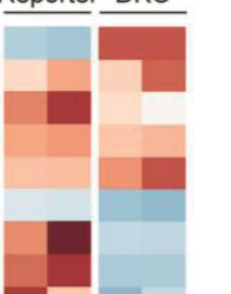

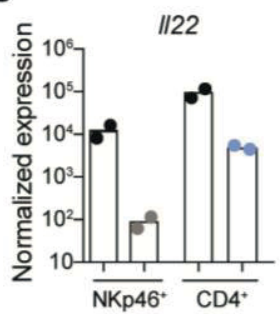

C DKO

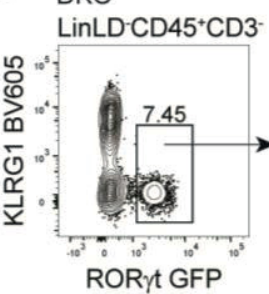

D

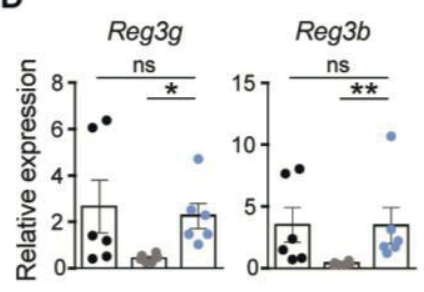

Figure 28. RORyt/T-bet-deficient ILCP produce IL-22 and promote intestinal barrier functions in adult mice. (A) Heatmap of bulk RNA-seq of ROR $\gamma t-G^{-} P^{+}$population sort-purified according to NKp46 or CD4 expression isolated from SI of 4-week-old mice. Selected gene transcripts are shown as Z-score. (B) Normalized expression values of $I / 22$ transcripts from bulk RNA-seq dataset and (C) IL-17A and IL-22 protein expression in SI of DKO mice determined by flow cytometry after 4h ex vivo restimulation using PMA/iono/IL23. Quantification of frequencies from three independent experiments $(n=11-16)$, mean \pm SEM and Kruskal-Wallis testing with Dunn's multiple correction. (D) Expression of Reg3g and Reg3b in intestinal epithelial compartment determined by quantitative PCR. Values are normalized to housekeeping genes Actb, Hprt and Gapdh. Each symbol represents an individual mouse. Data show mean \pm SEM, Kruskal-Wallis testing with Dunn's multiple correction, $\mathrm{n}=6$, see legend in $\mathrm{C}$.

Collectively, these data demonstrate that in the absence of T-bet and ROR $\gamma \mathrm{t}$, PLZF ${ }^{\text {hi }}$ ILCP with LTi signatures and residual Rorc promoter activity develop during embryonic life and persist into adulthood. To supplement the results from in vitro differentiation assays and to determine whether this effect was cell intrinsic in vivo, adoptive transfer experiments were conducted. To this end, irradiated $\mathrm{CD} 45.1^{+} \mathrm{CD} 90.2^{+} \mathrm{Rag}^{-1-} \mathrm{I} / 2 \mathrm{rg}^{/-}$recipient mice received progenitors derived from FL of CD90.1+ strains from reporter, RKO or DKO all expressing CD45.2. In order to deplete residual radio-resistant host ILCs (Gasteiger et al., 2015; Hanash et al., 2012; Robinette et al., 2017), host mice were treated bi-weekly with anti-CD90.2 antibody (schematic overview on experimental setup see Figure 8, page $\mathbf{2 5}$ in material and methods section). Four weeks after reconstitution, DKO progenitors efficiently engendered a population of GFPhi cells at similar frequencies as observed in reporter mice, equivalent to the phenotype observed ex vivo in adult mice (Figure 29). 


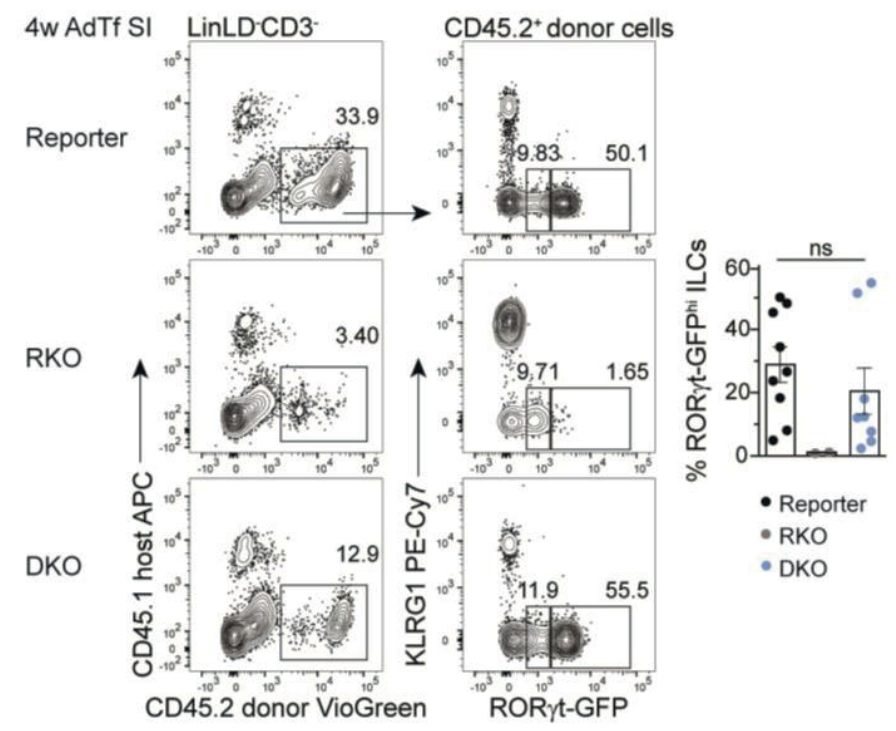

Figure 29. GFP+ ILCP are generated in in vivo adoptive transfer experimental setup. Representative flow cytometry in SI 4 weeks after adoptive transfer and quantification of frequencies of ROR $\gamma \mathrm{t}-\mathrm{GFPhi}$ ILCs as mean \pm SEM $(n=2-9)$, Kruskal-Wallis significance with Dunn's correction. Data are representative of 2-3 experiments.

Together, these results demonstrate that deficiency of T-bet and ROR $\gamma$ t facilitates the generation and accumulation of embryonic PLZFi ILCP, which can persist into adulthood. On the one hand, the absence of T-bet during ILCP differentiation enables expression of molecules crucial for LTi function thereby promoting LN formation, during embryonic development, while on the other hand, IL-22 production restores barrier functions during adulthood in DKO mice.

\subsection{LN development and accumulation of PLZFhi ILCP in DKO mice is promoted by ROR $\alpha$}

Since residual transcriptional activity at the Rorc locus was observed and GFPlo/hi expression was detected in both RKO and DKO mice, phenotypes were validated in alternative models of ROR $\gamma \mathrm{t}$ deficiency to exclude that LN generation and accumulation of ILCP in KO mice might be due to residual expression of ROR $\gamma t$ in DKO mice. $\operatorname{Rorc}(g t)^{G F P / G F P}(\mathrm{RKO})$ mice have been genetically modified by substitution of Rorc exon $1 \gamma$ t with a DNA sequence encoding GFP inserted directly

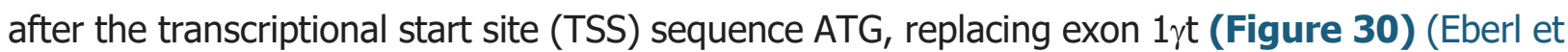
al., 2004). Hence, heterozygously bred mice report expression of ROR $\gamma \mathrm{t}$ by GFP, while in homozygously bred mice both alleles of Rorc exon $1 \gamma \mathrm{t}$ are replaced by GFP, resulting in loss of ROR $\gamma \mathrm{t}$ (knockout). To rule out that the observed phenotype is caused by functional activity of a potential residual protein transcribed from exon 3-11, Rorc(gt) ${ }^{4 e x 4}$ mice, which carry a conventional deletion in exon 4 of the Rorc locus (Sun et al., 2000) were crossed to Tbx21\% mice. Lack of exon 4 leads to disruption of the DNA-binding domain (DBD) of ROR $\gamma$ t (Figure 30). Moreover, $\operatorname{Rorc}(g t)^{f / f l}$ mice, in which Rorc exons 3 to 6 are floxed and cre recombinase activity deletes not only the DBD but also hinge and ligand-binding domain (LBD) regions of ROR $\gamma$ t (Figure 30) (Choi et al., 2016), were crossed to $T b \times 21^{f / f l}$ and $I / 7 r^{c r e / w t}$ mouse lines, enabling conditional deletion of T-bet as well as ROR $\gamma$ t exon 3-6 in IL-7R+ lymphocytes, including ILC progenitors from the CLP stage onwards. 


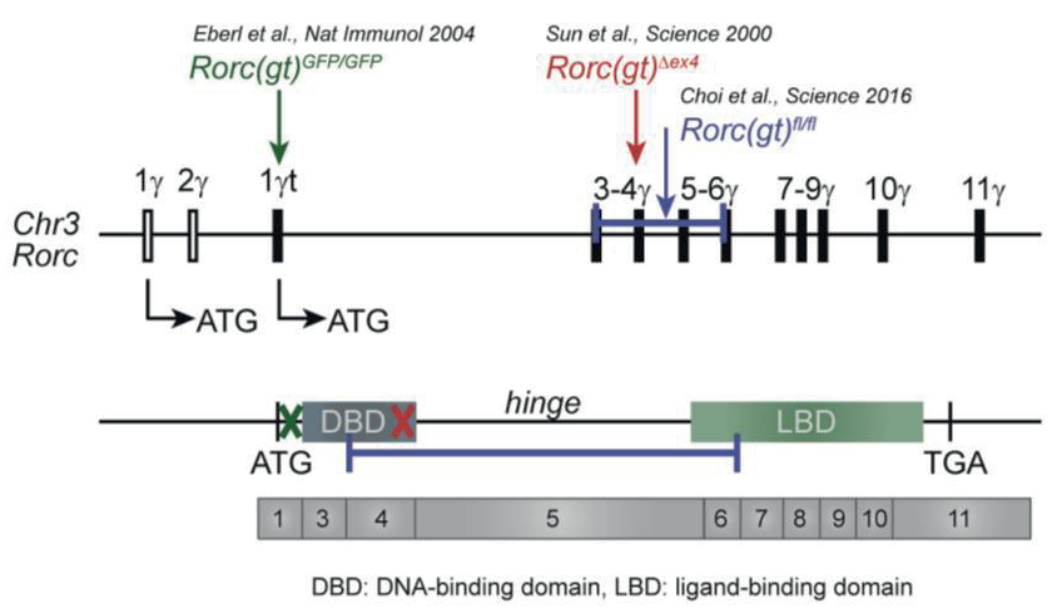

\begin{abstract}
Figure 30. Schematic overview of Rorclocus and Rorc deletions in mouse models. In $\operatorname{Rorc}(g t)^{G F P / G F P}$ mice GFP is inserted directly after the transcriptional start site ATG of exon $1 \gamma \mathrm{t}$ while $\operatorname{Rorc}(g t)^{\text {tex4 }}$ have a conventional deletion in exon 4 that encodes for the DNA-binding domain (DBD). In $\operatorname{Rorc}(g t)^{f l / f l}$ mice exon 3-6 are surrounded by floxed regions and under cre-recombinase activity delete $\mathrm{DBD}$, hinge and ligand-binding domain (LBD) of ROR $\gamma$ t.
\end{abstract}

Notably, thymic disruption of T cell development typically associated to ROR $\gamma$ t deficiency (Guo et al., 2016; He et al., 2000; Sun et al., 2000) was observed in both ROR $\gamma$ t single-deficient mouse models (Rorc(gt) ${ }^{G F P / G F P}$ and $\operatorname{Rorc}(g t)^{4{ }^{1 e x} 4}$ mice) and could not be rescued in ROR $\gamma \mathrm{t} / \mathrm{T}$-bet doubledeficient $\left(\operatorname{Rorc}(g t)^{G F P / G F P} \times \operatorname{Tb} \times 21^{\%}\right.$ and $\left.\operatorname{Rorc}(g t)^{\operatorname{Mex} 4} \times \operatorname{Tb} \times 21^{-/}\right)$mice. Likewise, thymic compartments were similarly impaired in $I / 7 r^{c r e / w t} \times \operatorname{Rorc}(g t)^{f / f f} \times T b \times 21^{f / f l}$ mice, altogether corroborating the absence of functional ROR $\gamma$ t activity (Figure 31).

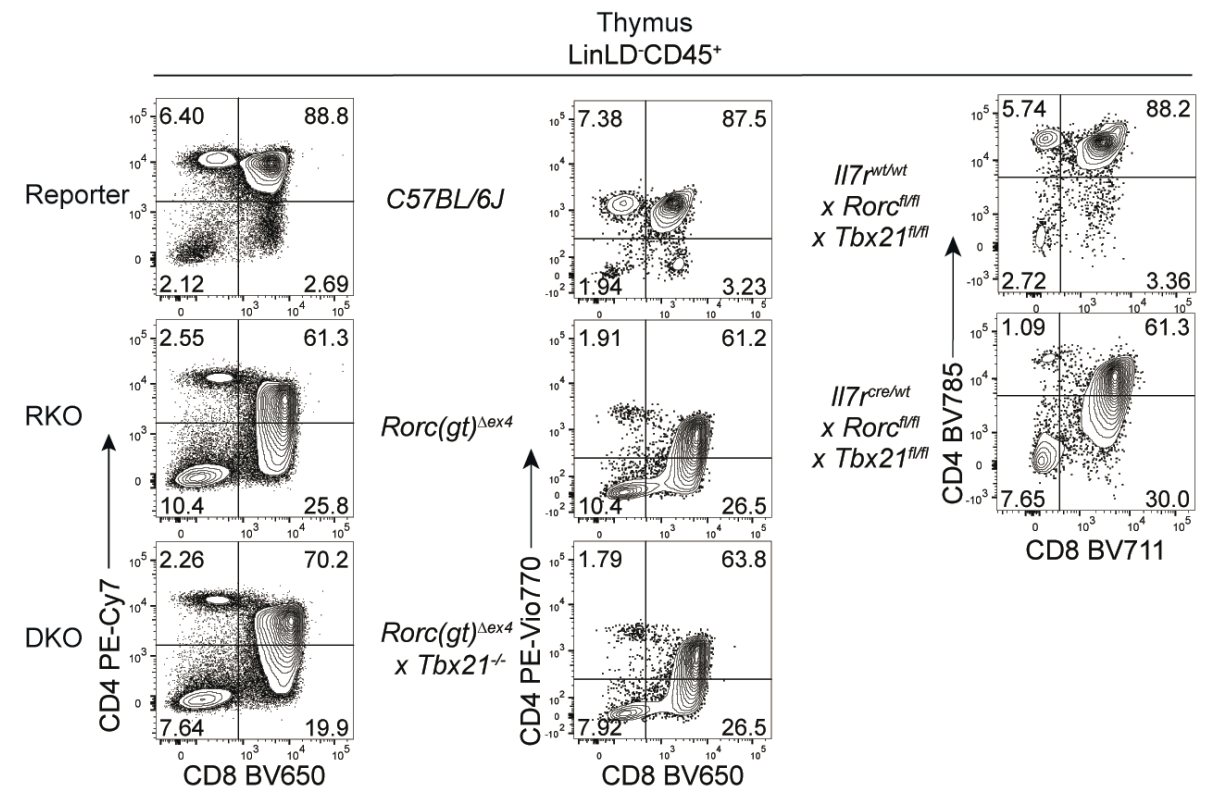

Figure 31. Thymic $\mathrm{CD}^{+} \mathrm{CD}^{+}$compartments are not restored in ROR $\mathbf{r}$ /T-bet double-deficient mouse models. Flow cytometry representative plots of thymi from 4-week-old mice of indicated mouse strains. 
Markedly, both additional models of concomitant ROR $\gamma$ t and T-bet deletion phenocopied $\operatorname{Rorc}(g t)^{G F P / G F P} \times$ Tbx21\%- mice, resulting in presence of LN which were absent in the ROR $\gamma \mathrm{t}$ singledeficient counterparts ( $\operatorname{Rorc}\left(g t^{\mathrm{eex} 4}\right.$ and $I / 7 r^{\mathrm{cre} / \mathrm{wt}} \times \operatorname{Rorc}(g t)^{f / f l}$ mice) (Figure $32 \mathrm{~A}$ and B). In support of this phenotype, PLZFi ILCP accumulated in $\mathrm{mLN}$ of both additional mouse strains lacking ROR $\gamma$ t and T-bet (Figure $32 \mathrm{C}$ and D), matching the observations in $\operatorname{Rorc}(g t)^{G F P / G F P} x$ Tbx $21^{-1-}$ DKO versus Rorc(gt) $)^{G F P / w t}$ reporter mice.
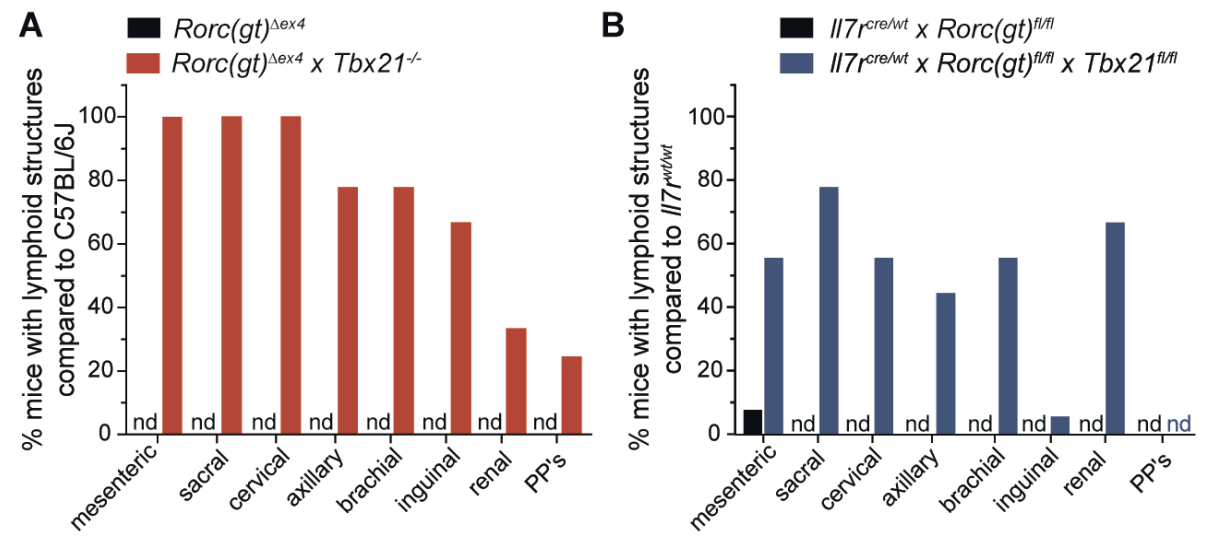

。
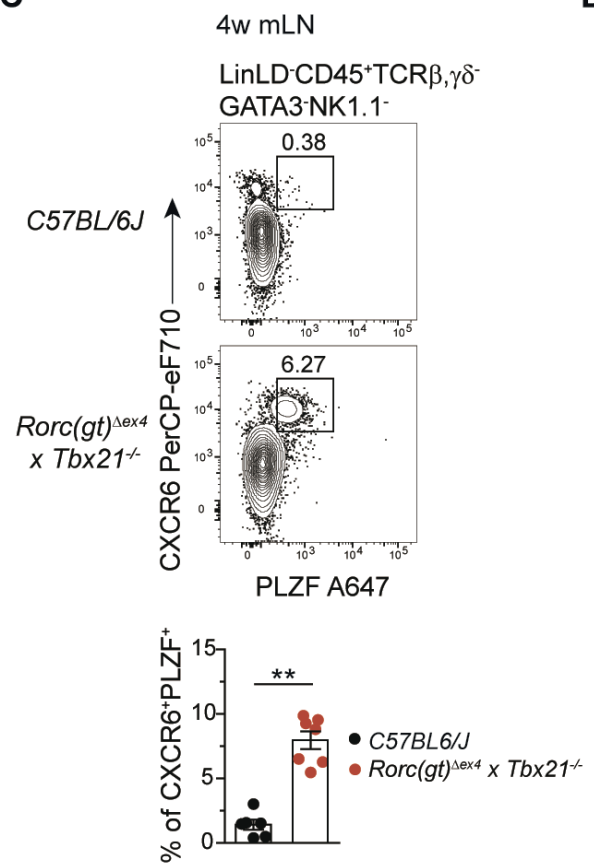

D

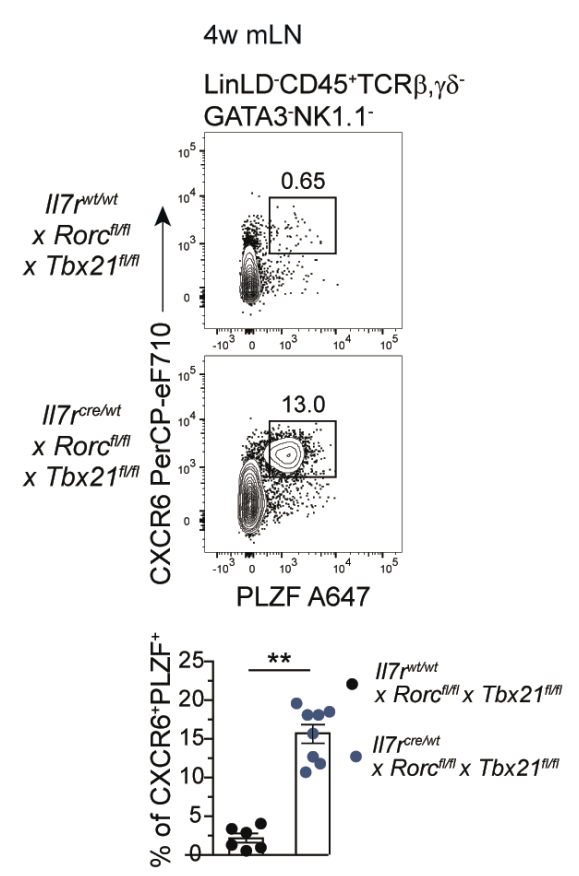

Figure 32. LN formation and accumulation of PLZF ${ }^{+}$ILCP is independent of DNA- and ligand-binding domains of ROR $\gamma \mathrm{t}$ in the absence of T-bet. (A, B) Quantification $(n=12)$ of frequencies of mice with lymphoid structures compared to control mouse strain in adult animals. PP's, Peyer's patches; nd, not detected. (C, D) Flow cytometry representative plots of $\mathrm{mLN}$ from 4-week-old mice of indicated mouse strains. Quantification of frequencies from two to three independent experiments as mean \pm SEM $(n=6-8)$, Mann-Whitney-U testing.

Together, these results demonstrate that, in the absence of T-bet, LN formation and accumulation of PLZFi ILCP with LTi signatures occur irrespective of ROR $\gamma$ t TSS ATG, DBD, hinge 
and LBD regions, indicating that acquisition of LTi functions, but not full ILC3 maturation or thymic T cell development, can happen independently of ROR $\gamma$ t.

Finally, to assess whether other transcription factors promote acquisition of LTi profile and LN formation in the absence of ROR $\gamma$ t and T-bet, the importance of ROR $\alpha$ (closely related to ROR $\gamma \mathrm{t}$ ) was analysed, which has been shown to cooperatively regulate Th17 genes conjointly with RORyt (Castro et al., 2017; Sundrud and Rao, 2008; Yang et al., 2008a). In the E18.5 scRNA-seq dataset, Rora was found to be highly expressed in ILCP from all mouse strains (Figure 33 A). To test whether ROR - could substitute absence of ROR - t and account for generation of ILCP displaying LTi activity $I / 7 r^{c r e / w t} \times \operatorname{Rorc}(g t)^{f / f l} \times \operatorname{Tb} \times 21^{f / f l}$ mice were crossed to Rora ${ }^{f / f l}$ mice and

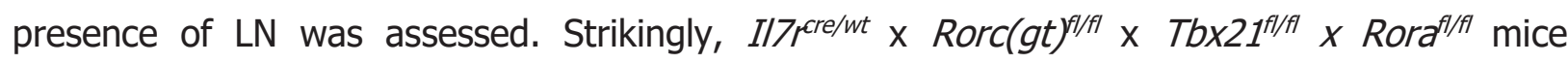
showcased complete absence of all LN (Figure 33 B). Along this line, intestinal PLZF ${ }^{\text {hi }}$ ILCP did not generate in triple-floxed mice compared to double-floxed animals (Figure $33 \mathbf{C}$ ).

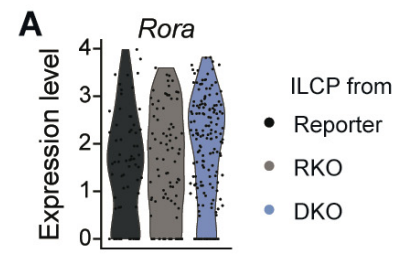

B

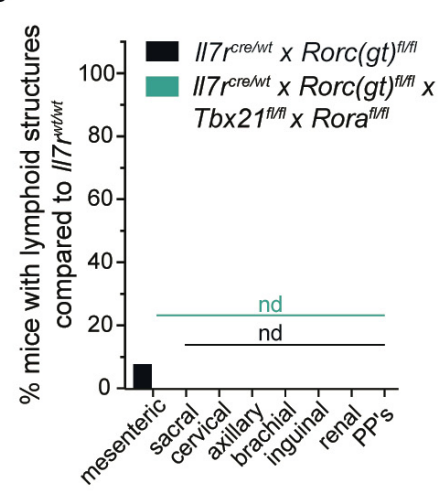

C $\quad 4 \mathrm{wSI}$

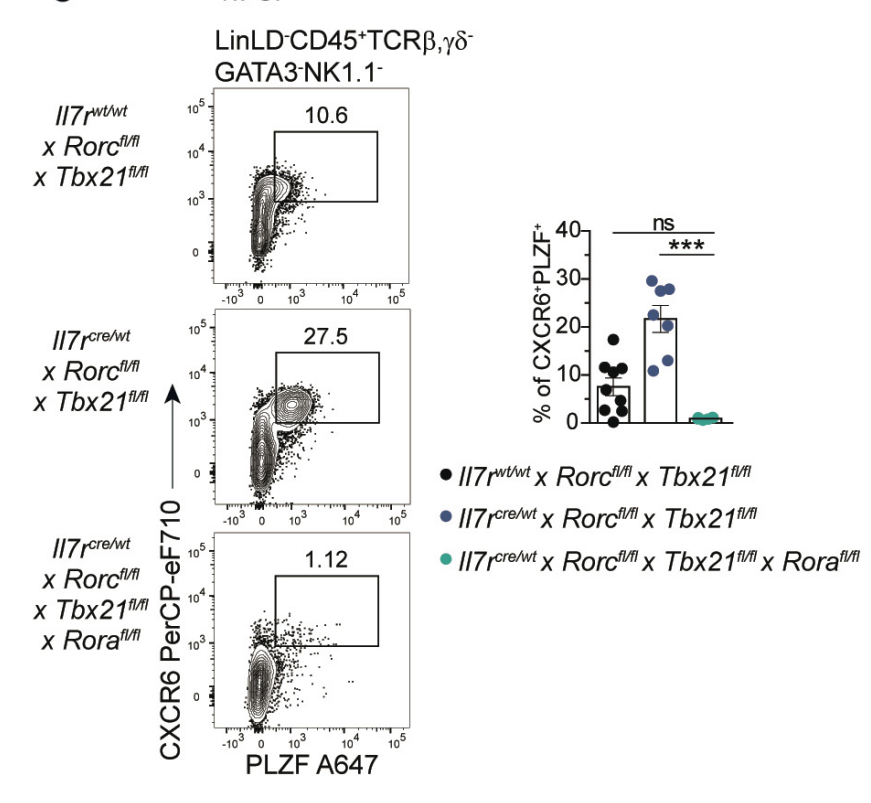

Figure 33. ROR $\alpha$ promotes LN development and PLZF ${ }^{+}$ILCP accumulation in DKO mice. (A) Violin plot of Rora expression within ILCP of designated mouse strains from E18.5 scRNA-seq dataset. (B) Quantification $(n=12)$ of frequencies of mice with lymphoid structures compared to control mouse strain in adult animals. PP's, Peyer's patches; nd, not detected. (C) Flow cytometry representative plots of SI from 4-week-old mice of indicated mouse strains. Quantification of frequencies from two to three independent experiments as mean \pm SEM $(n=8-9)$, Kruskal-Wallis testing with Dunn's multiple correction.

Altogether, the present study reveals a complex interplay between T-bet, ROR $\gamma \mathrm{t}$ and ROR $\alpha$ in regulating fetal ILCP differentiation and unravel a previously unrecognized role for T-bet and $\mathrm{ROR} \alpha$ in LN organogenesis during embryonic life. 


\section{Discussion}

The fetal intestine holds mature ILC subsets and immature progenitors

ILCs are among the first lymphocytes to appear during ontogeny to seed their respective organs and tissues even before cells of the acquired immune system are generated. Nevertheless, apart from broad description in the intestine of adult animals also at single-cell level (Gury-BenAri et al., 2016; Hernández et al., 2018), their transcriptomic profile in the fetus has not been intensively studied to date. To identify not only the embryonic transcriptional profile of ILCs but also to characterize their phenotype in parallel, proteogenomic single-cell RNA sequencing was performed that allows to accurately understand fetal ILC diversity. Since the development and homeostasis of ILCs depends on stromal IL-7, CD127 has evolved as a defining marker for all ILCs. However, CD127 expression varies throughout ILC subsets and tissues. In the current study, all members of the ILC family were effectively sorted by using CD127 and additionally CD122 as ILC surface markers, furthermore revealing progenitor populations that could be defined as CLPS and ILCPs within the isolated tissue. Intriguingly, accumulating evidence suggests that local pools of tissue-resident ILCs originate from circulating naive progenitor cells that mature within the tissue (Lim et al., 2017; Nagasawa et al., 2019; Oherle et al., 2020; Zeis et al., 2020). On this matter, Locksley and colleagues have previously described a population of Arg1 ${ }^{+}$ILCP in fetal intestine of Arg1 reporter mice (Bando et al., 2015), probably largely overlapping with the ILCP detected in this work, which also expressed Arg1. Furthermore, CLP and ILCP populations have also been recently reported in peripheral LN anlagen at E13.5 and E14.5 (Simic et al., 2020), suggesting that FL CLP and ILCP might seed these sites to mature locally. Altogether, these results highlight the embryonic intestine as an active site of differentiation in situ during fetal development.

Interestingly, while ILC2 bifurcated at an early stage, trajectory analysis revealed a common lineage development of ILC3 and ILC1/NK. Similar observations were reported in BM-derived progenitors where ILC2 rapidly commit to lineage while putative ILC1, ILC3 and NK progenitors display heterogeneous TF expression with many more transitional states (Walker et al., 2019). The current study underlines this concept and similarly suggests a greater common transcriptional regulation of ILC3/ILC1 lineages in embryonic ILC differentiation. While ILCP differentiation was highly influenced in mice deficient for ROR $\gamma$ t and/or T-bet, ILC2 differentiation remained unaffected in the used KO models, pinpointing towards a lineage differentiation program different to ILC3/ILC1 lineages.

\section{Fetal ILC3 are heterogeneous and display T-bet expression}

Embryonic LTi cells were described and considered a rather homogenous population with the central function to organize LN formation. In mature mice, it is well established that ROR $\gamma \mathrm{t}$ and 


\section{Discussion}

T-bet are co-expressed within ILC3 where they cross-regulate each other. Remarkably, a certain heterogeneity within the transcriptome of E18.5 intestinal ILC3 was detected in the present work. Besides LTi cells, the emergence of an unappreciated T-bet ${ }^{+}$ILC3 subset was observed. Using T-bet ZsGreen reporter mice, the ILC compartments were dissected in a kinetic fashion in the developing intestine and a T-bet ${ }^{+} \mathrm{CD}^{+}$population was found as early as E14.5 displaying some phenotypic similarities with classical LTi cells. While the current data confirm the prenatal absence of ILC3 expressing NKp46 protein, T-bet transcripts and protein expression starts already as early as E14.5 during embryonic development. The quantitative expression of the two lineage-defining TFs ROR $\gamma$ t and T-bet may alter their reciprocal balance, influencing phenotype and plasticity of ILC subsets. Indeed, T-bet, GATA3 and ROR $\gamma$ t haplo-insufficiencies have been reported to affect marker expression and functions (Klein Wolterink et al., 2013; Rankin et al., 2016; Walker et al., 2019; Zhong et al., 2016). Contrary to the prevailing view, the data obtained here indicate that, acquisition of T-bet and generation of plastic transcriptional states by loss of ROR $\gamma$ t or T-bet, as investigated using ROR $\gamma t-F M$ and T-bet-FM reporter mice, is not a phenomenon linked to exposure to inflammatory signals postnatally, but can occur already prenatally. Together, these data indicate a prenatal divergence of group 3 ILC subsets with imprinted transcriptional programs, independent of colonization of the intestine with commensal microbiota. The observed transcriptional states in the fetal gut might instead result from other environmental cues such as for instance cellular interactions or local cytokine milieus in the developing tissue. As described in the introduction section 1.3.3, environmental signals influencing ROR $\gamma$ t expression in fetal LTi cells have been described, such as maternal-derived RA, indicating a direct regulation by localized metabolites (Van De Pavert et al., 2014). It has also been shown in ILC3 of adult mice (see also section 1.3.2), that T-bet can be induced by Notch signaling to generate NKp46+ subsets (Rankin et al., 2013; Verrier et al., 2016). Such local availabilities of functional molecules or receptors in the evolving intestinal tissue could represent plausible factors influencing TF expression and/or their balance prenatally.

T-bet has furthermore been shown to bind and activate numerous genes like CXCr3, Ccr5, Itgal and Icam1, thereby controlling $\mathrm{T}$ cell transcriptional programs and determining lymphocyte homing (Jenner et al., 2009; Lord et al., 2005; Matsuda et al., 2007). A similar influence of T-bet on regulation of chemokine receptor expression in ILC3 might actively control migratory features to sites other than LN anlagen suggesting that heterogenic ILC3 might locate to specific environments. Interestingly, reduced expression of CXCR5, CCR6 and integrin $\alpha_{4} \beta_{7}$ was observed in ILC3 that co-expressed T-bet (Figure 15 F and G), potentially affecting their homing properties to specific niches.

Notably, further heterogeneity besides type 1-like ILC3 was observed, with one cluster displaying enhanced transcriptional circuits associated to MHCII molecules. These ILC3 emerged in the 
absence of $\mathrm{CD}^{+} \mathrm{T}$ cells that will only generate after birth. In adult mice, selective ablation of MHCII on ILC3 impaired adaptive T and B cell responses, weakened tolerance to commensals and induced intestinal inflammation (Von Burg et al., 2014; Hepworth et al., 2013, 2015). How this relates to embryonic MHCII-expressing ILC3 and what role tolerance versus immunity plays at this phase in ontogeny remains to be seen. With regards to ILC3-mediated tolerance unrelated to microbiota, embryonic LTi cells in the thymus were shown to provide RANKL stimulation to medullary thymic epithelial cells (mTECs) to induce expression of autoimmune regulator (Aire) (Roberts et al., 2012). Along this line, Aire+ ILC3 with potent antigen-presenting features were found in peripheral LN of adult mice, where those cells could be rarely detected at birth, numbers peaked at the time of weaning and declined thereafter (Yamano et al., 2019). In this context, it will be interesting to clarify whether $\mathrm{MHCII}^{+}$ILC3 with antigen-presenting potential are involved in establishment of early $T$ cell tolerance in the developing intestinal environment prenatally.

Remarkably, MHCII expression by ILC3 was shown to be regulated by the pIV promoter elements of the master TF for MHCII transactivator or CIITA in an IFN $\gamma$-dependent manner (Hepworth et al., 2015). Whether IFN $\gamma$ could serve as a polarizing signal determining the heterogeneity towards MHCII-expressing ILC3 in the embryonic intestine is an interesting question that remains to be addressed in future studies.

\section{Specified ILC3 subsets might indicate a layered ontogeny}

Multiple studies over the past years have revealed that diverse lymphoid subsets are exclusively produced during the embryonic to neonatal period such as B-1a B cells locating to peritoneal and pleural cavities or certain subsets of $\gamma \delta$ T cells residing in gut and skin (Böiers et al., 2013; Kobayashi et al., 2014; Yoshimoto et al., 2011, 2012). In this regard, it is progressively becoming clearer from previous mouse studies that ILCs arise from distinct waves during development, a term called layered ontogeny (Oherle et al., 2020; Schneider et al., 2019). Interestingly, in the present study, it was observed that early CD4 ${ }^{+} \mathrm{LTi}^{-}$cells were CCR6 ${ }^{-}$and that CCR6 ${ }^{+} \mathrm{CD}^{-}$ILC3 $^{-}$ started to establish only between E16.5 and E18.5, suggesting diverse waves of ILC3 differentiation. Considering that, during embryonic development distinct waves of hematopoietic progenitors emerge prior to the generation of HSCs, giving rise to a series of transient progenitors, it is conceivable that ILC3, especially early LTi cells, might in part originate from different layers to fulfill the immediate needs of the developing embryo. The first wave is initiated at E7 from endothelial cells that undergo Endothelial to Hematopoietic cell Transition (EHT), localizing to the yolk-sac and generating progenitors with potential to give rise to primitive erythrocytes, macrophages and megakaryocytes (Moore and Metcalf, 1970; Palis et al., 1999; Tober et al., 2007). A second wave with broader myeloid potential emerging after establishment of circulation locating to the blood vessel of the yolk sac from E8, to generate erythro-myeloid progenitors (EMPs) (McGrath et al., 2011, 2015b). Only the third final wave, produces cells with 


\section{Discussion}

lymphoid potential (LMPPs, lymphoid- and myeloid-primed progenitors) around E10 (Böiers et al., 2013; Ohmura et al., 1999, 2001). At this timepoint, the first HSC are detected within in the aorta-gonad-mesonephros (AGM) region as well as in vitelline and umbilical vessels as extraembryonic sites (De Bruijn et al., 2000; Gordon-Keylock et al., 2013; Kumaravelu et al., 2002; Müller et al., 1994). Later in ontogeny, HSCs mobilize into FL before emigrating into the BM just before birth. By analyzing several mouse lines with time-specific cre expression, van de Pavert et al. describe a detailed view of ILC3 ontogeny (Simic et al., 2020) and suggest that hemogenic progenitors appear from the third wave of hematopoiesis explicitly in early embryonic hemogenic endothelium, giving rise to proliferating precursor populations in FL. They furthermore propose that embryonic LTi cells are replaced by HSC-derived LTi cells in adults. In another study using Vavere $x$ Gata ${ }^{f / f l}$ mice it was shown that ILC1/2/3 do not develop where Vav ${ }^{c r e}$ is active at the HSC stage (Yagi et al., 2014). Surprisingly, Vav ${ }^{\text {re }} x$ Gata ${ }^{f / f l}$ mice do not generate $\mathrm{CD} 127^{+} \alpha_{4} \beta_{7}^{+}$ILCP in adult BM; however such progenitors could be detected in E15.5 FL indicating that these might derive from an early wave before cre-excision. Intriguingly, Vavre $x$ Gata $^{f / f f}$ mice harbor a small population of $\mathrm{CD} 4^{+} \mathrm{LTi}$ cells in adult SI, possibly originating from the embryonic progenitors, but do not develop LN (Yagi et al., 2014). Why these mice fail to generate LN is not clarified. It is thinkable, that quantity of LTi cells might be crucial but numbers of embryonic LTi cells were not analyzed in their study.

Whether ILCs originating from these diverse waves of differentiation might differ in their functions, or whether they just need to be localized to specific sites in time and space to accomplish complex developmental phases of tissue or organ development remains to be clarified in future studies. Upcoming technical advances in lineage tracing together with in-depth single cell transcriptional and epigenetic technologies will help to discover new insights into how sequential layers during ontogeny contribute to different lineage progeny and decipher their functional heterogeneity.

\section{ROR $\gamma$ t counteracts T-bet during fetal ILC differentiation}

With the intention to delineate the role of T-bet in ILC3 lineage specification during embryogenesis, it was found that lack of T-bet restored the development of peripheral LN, typically absent in ROR $\gamma$ t-deficient mice. This phenotype was recapitulated in three independent mouse models with different Rorc locus disruptions, pinpointing a redundant role for DNA- and LBD-domains as well as hinge region of ROR $\gamma \mathrm{t}$ for LN formation in the absence of T-bet. Notably, LN formation in all double knockout strains was associated with the appearance and accumulation of an ILCP population expressing PLZF that, due to absence of ROR $\gamma$ t and T-bet, is likely not able to differentiate towards mature ILC lineages. Accumulation of PLZFhi ILCP was not accompanied with increased proliferation but might be linked to increased survival, as suggested by elevated expression of the anti-apoptotic molecule $B C l 2$, whose transcripts were enriched among DKO 
ILCP (Figure 23 F). It was previously shown in Zbtb16 GFPcre FM mice that PLZF transiently marks a FL and BM ILCP, which has lost LTi potential, while mature ILCs are largely negative for PLZF (Constantinides et al., 2014). The data here confirm high enrichment of PLZF expression in ILCP from embryonic intestine and $\mathrm{mLN}$ anlage but demonstrate that Zbtb16 expression is also present, albeit at a lower level, in fetal ILCs especially within ILC3/LTi cells, in line with a recent report by McKenzie et al. (Walker et al., 2019). Using scRNA-seq this study furthermore describes a CXCr5-expressing ILCP in adult BM, and together with data obtained in another study using bulk RNA-seq, they propose CXCR5 as a useful marker to identify ILCP (Harly et al., 2018; Walker et al., 2019). Besides CXCr5 transcripts and protein, in the present study expression of LTi functional molecules such as RANKL and $L T \alpha_{1} \beta_{2}$ heterotrimer was detected within embryonic tissue-derived ILCP, preceding expression of Rorcthat subsequently allows maturation to full LTi cell phenotype. These data suggest that ROR $\gamma$ t is important to drive full differentiation of the ILCP towards the ILC3 lineage but seems redundant for the acquisition of central LTi molecules and related functions, such as LN development. A model of mixed lineage priming (Ishizuka et al., 2016; Walker et al., 2019) with transient upregulation of multiple master TFs has been proposed in differentiating ILCP isolated from E15.5 FL or adult BM, a process also observed here in tissue-derived ILCP. However, upregulation of LTi signatures within these progenitors only manifested in the absence of T-bet and did not emerge in ROR $\gamma$ t single deficient mice, where ILCP preferentially differentiate towards ILC1 (and ILC2). Thus, this indicates that ROR $\gamma \mathrm{t}$ has a central role in counteracting T-bet-driven transcriptional programs highlighting the importance of a balanced expression of these two TFs in the regulation of ILC3/ILC1 lineage maturation not only postnatally, but also prenatally.

\section{ROR $\gamma$ t-independent restoration of IL-22 in the absence of T-bet}

ILC3 do not only play a crucial role in fetal development of $L N$, but also in sustaining intestinal homeostasis postnatally by their production of IL-22. In the absence of ROR $\gamma$ t and T-bet, PLZFhi ILCP persisted in adult $\mathrm{mLN}$ and intestine, and promoted epithelial barrier functions via restored IL-22 production. While the role of ROR $\alpha$ in restoring IL-22 from ILC3 in the absence of ROR $\gamma \mathrm{t}$ and T-bet was not directly ascertained in this study, this has been recently shown in a study accepted for publication (Fiancette et al., 2021, personal communication). Moreover, additional TF might contribute to this effect. For example, the TF Ahr has been shown to be critical for IL-22 production by ILC3 (Kiss et al., 2011; Qiu et al., 2012) but also GATA3 binds to the I/22 promoter to regulate production within ILC3 (Zhong et al., 2016). Hence, various other factors, also found here to be expressed in ILCP, may compensate for the lack of ROR $\gamma$ t to foster the production of IL-22. Interestingly, analysis of patients carrying bi-allelic Rorc loss-of-function mutations revealed absence of IL-17-producing lymphocytes but residual IL-22 producers suggesting similar compensatory pathways in humans (Okada et al., 2015). 


\section{Discussion}

ROR $\gamma$ t and ROR $\alpha$ act to limit T-bet-driven programs in ILCP

Finally, in regards to which other TF might compensate and promote expression of LTi molecules, $\mathrm{ROR} \alpha$ was reported to synergistically regulate Th17 genes together with ROR $\gamma \mathrm{t}$ and has furthermore been described to stably maintain ILC3 lineage (Castro et al., 2017; Lo et al., 2016, 2019; Sundrud and Rao, 2008; Yang et al., 2008b). New data show that ROR $\alpha$ binds to an enhancer element within the Rorc locus reinforcing ROR $\gamma$ t transcriptional programs and crucially stabilizing Th17 lineage maintenance (Lee et al., 2020). Likewise, ROR $\alpha$ is suggested to essentially preserve ILC3 fate during $S$. typhimurium infection and in its absence ILC3 display enhanced core ILC1 genes proposing conversion into ex ILC3 (Lo et al., 2019). While ROR $\alpha$ expression is a common feature of all ILC subsets (Halim et al., 2012; Wong et al., 2012), an additional study also predicted ROR $\alpha$ to repress ILC1/NK gene expression under homeostatic conditions (Pokrovskii et al., 2019). This led to the assumption that ROR $\alpha$ could potentially compensate for the loss of ROR $\gamma$ t in T-bet-deficient ILCP and endorse expression of LTi cell-

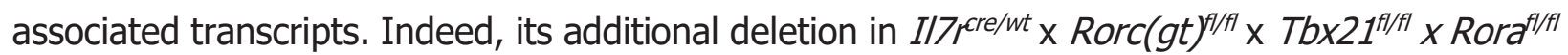
mice abolished LN development as well as accumulation of PLZF ${ }^{\text {hi }}$ ILCP. Collectively, these findings demonstrate a new role for ROR $\gamma \mathrm{t}$ in counteracting T-bet driven differentiation of embryonic ILCP and reveal ROR $\alpha$ as a crucial regulator of ILCP differentiation and early acquisition of LTi signatures. A strict balance of ROR $\gamma$ t and T-bet in differentiating ILCP governs the ILC3/ILC1 equilibrium, while ROR $\alpha$ sustains PLZF ${ }^{\text {hi }}$ ILCP accumulation and functions before and after birth.

Altogether, the results provided in this study define a novel role for T-bet during fetal ILC3 differentiation and LN generation and unveil a previously unrecognized embryonic ILC3 heterogeneity complementing and advancing the knowledge of transcription factor networks yet described in adult ILC lineages.

\subsection{Concluding remarks and open questions}

Collectively, this work provides new insights into transcriptional regulations within embryonic ILC and identify TF networks crucial for formation of LN. The key findings are summarized below.

- Single-cell sequencing of fetal intestinal cells identifies mature ILCs and progenitors

- T-bet $^{+}$ILC3 are already present in the E14.5 intestine, liver and mLN anlage

- T-bet deficiency rescues LN organogenesis in ROR $\gamma$ t-deficient mice and is associated with accumulation of a PLZF ${ }^{\text {hi }}$ ILCP population with LTi functions

- $\quad$ PLZF ${ }^{\text {hi }}$ ILCP persist in the adult $\mathrm{mLN}$ and intestine and promote epithelial barrier functions via restored IL-22 production 
- LN development and accumulation of PLZFhi ILCP with LTi functions in mice deficient for ROR $\gamma$ t and T-bet is dependent on ROR $\alpha$

In view of these novel findings, several open questions remain and might direct potential future subjects of investigation, for example:

Which signals drive early acquisition of T-bet during fetal ILC differentiation (IL-12, IFN $\gamma$, Notch)? Where do CLPS and ILCPs localize in the developing intestine? Is there a special niche with distinct environmental settings and special cellular components? Future improvements of techniques such as spatial transcriptomics will help to decipher this issue.

The understanding of the programmed development of secondary lymphoid organs has progressively increased over the last decade and the identified LTi cells interacting with stromal cells as key players herein. These processes are orchestrated by a variety of chemokines, cytokines and cell-cell contacts; however, the exact mechanisms and signals needed for development of the various distinct lymphoid structures are not clarified in detail at current. In light of the re-occurrence of most but not all LN in ROR $\gamma \mathrm{t} / \mathrm{T}$-bet-DKO mice, what exact role do ILCP with LTi activity play in the generation of specific LN? Could separate waves of ILC3 differentiation colonize individual LN anlagen? May different ILC3 subsets home to specific sites of LN formation?

Human ILC poiesis is less well characterized and most studies use CD34+ CLP-like hematopoietic progenitor cells which can be further delineated by a defined a set of surface markers. Committed ILC3 precursors have been described in human tonsil and intestinal lamina propria and a subset of IL-17-producing fetal LTi cells was also identified. Are intermediate stages displaying expression of PLZF and LTi genes also present in humans? 


\subsection{Graphical summary}

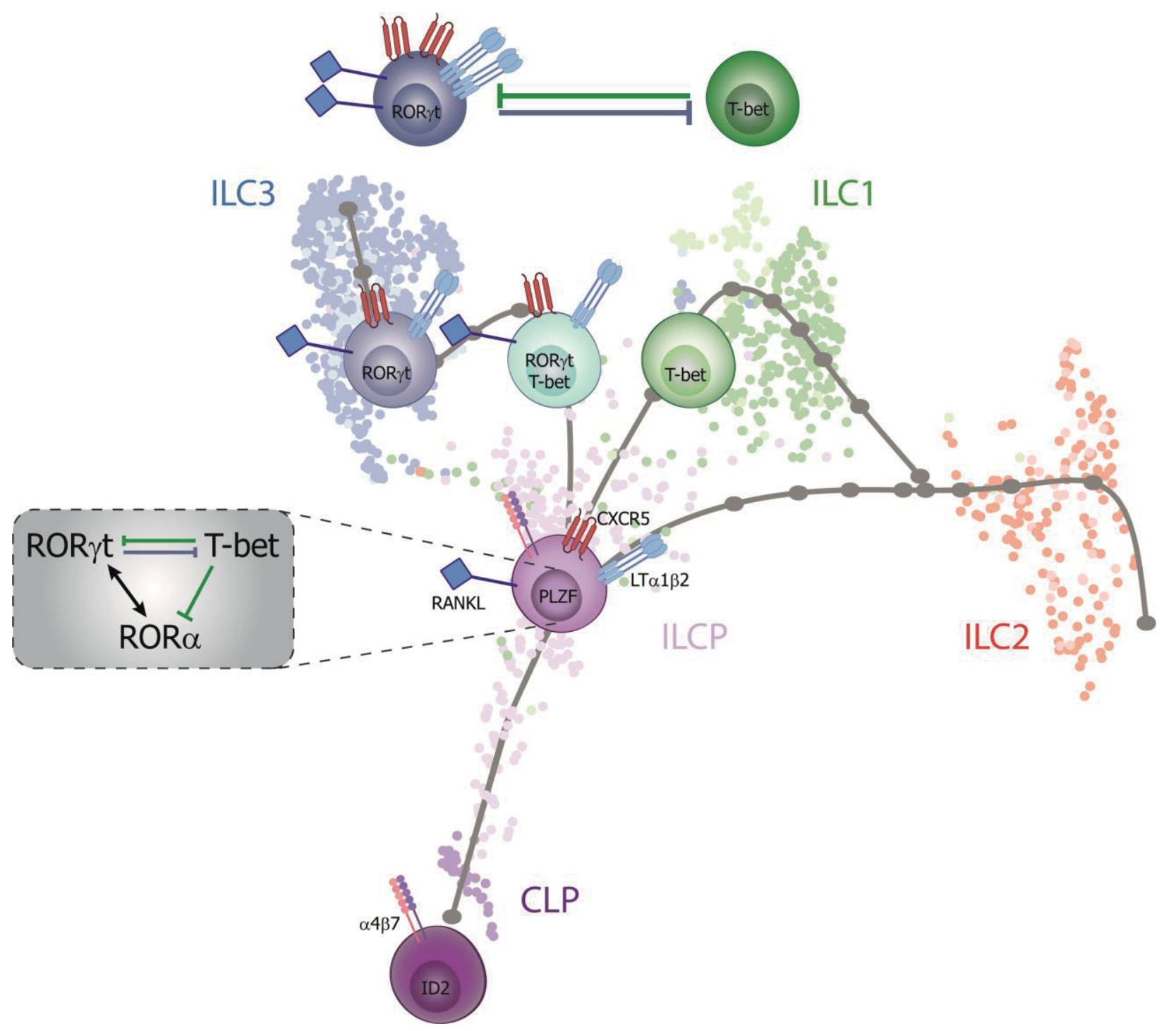

Figure 34. Schematic overview on transcription factor networks regulation during ILC differentiation. Differentiation of common lymphoid progenitors (CLP) towards innate lymphoid cell progenitors (ILCP) is marked by upregulation of transcription factors ID2 and PLZF. ROR $\alpha$ promotes expression of lymphoid tissue inducer (LTi) cell activity-associated molecules such as CXCR5, RANKL as well as the heterotrimer LT $\alpha_{1} \beta_{2}$ in differentiating ILCP. At this stage, a phase of multilineage priming with upregulation of various lineage-defining transcription factors eventually determines cell progeny fate. The two orphan nuclear receptor transcription factors ROR $\gamma \mathrm{t}$ and ROR $\alpha$ synergistically regulate gene expression, whereas, expression of T-bet counteracts ROR $\alpha / \gamma$ t-driven signatures and induces a type 1associated transcriptional program that antagonizes LTi-related gene transcription. Thus, the absence of T-bet skews the differentiation fate of fetal ILCs and promotes accumulation of PLZF ${ }^{+}$ILCP expressing central LTi molecules. In mature ILC3, ROR $\gamma$ t is crucial in counteracting the suppressive effects of T-bet. 


\section{References}

A., P., Eraslan, G., Virshup, I., and Gigante, S. (2020). theislab/anndata2ri v1.0.4.

Adachi, S., Yoshida, H., Kataoka, H., and Nishikawa, S.I. (1997). Three distinctive steps in Peyer's patch formation of murine embryo. Int. Immunol. 9, 507-514.

Aliahmad, P., De La Torre, B., and Kaye, J. (2010). Shared dependence on the DNA-binding factor TOX for the development of lymphoid tissue-inducer cell and NK cell lineages. Nat. Immunol. 11, 945-952.

Ansel, K.M., Ngo, V.N., Hyman, P.L., Luther, S.A., Förster, R., Sedgwlck, J.D., Browning, J.L., Upp, M., and Cyster, J.G. (2000). A chemokine-driven positive feedback loop organizes lymphoid follicles. Nature 406, 309-314.

Bando, J.K., Liang, H.E., and Locksley, R.M. (2015). Identification and distribution of developing innate lymphoid cells in the fetal mouse intestine. Nat. Immunol. 16, 153-160.

Banks, T.A., Rouse, B.T., Kerley, M.K., Blair, P.J., Godfrey, V.L., Kuklin, N.A., Bouley, D.M., Thomas, J., Kanangat, S., and Mucenski, M.L. (1995). Lymphotoxin-alpha-deficient mice. Effects on secondary lymphoid organ development and humoral immune responsiveness. J. Immunol. 155.

Bar-Ephraim, Y.E., Cornelissen, F., Papazian, N., Konijn, T., Hoogenboezem, R.M., Sanders, M.A., Westerman, B.A., Gönültas, M., Kwekkeboom, J., Den Haan, J.M.M., et al. (2017). Cross-Tissue Transcriptomic Analysis of Human Secondary Lymphoid Organ-Residing ILC3s Reveals a Quiescent State in the Absence of Inflammation. Cell Rep. 21, 823-833.

Basu, R., O'Quinn, D.B., Silberger, D.J., Schoeb, T.R., Fouser, L., Ouyang, W., Hatton, R.D., and Weaver, C.T. (2012). Th22 Cells Are an Important Source of IL-22 for Host Protection against Enteropathogenic Bacteria. Immunity 37, 1061-1075.

Bernink, J.H., Peters, C.P., Munneke, M., Te Velde, A.A., Meijer, S.L., Weijer, K., Hreggvidsdottir, H.S., Heinsbroek, S.E., Legrand, N., Buskens, C.J., et al. (2013). Human type 1 innate lymphoid cells accumulate in inflamed mucosal tissues. Nat. Immunol. 14, 221-229.

Bernink, J.H., Krabbendam, L., Germar, K., de Jong, E., Gronke, K., Kofoed-Nielsen, M., Munneke, J.M., Hazenberg, M.D., Villaudy, J., Buskens, C.J., et al. (2015). Interleukin-12 and 23 Control Plasticity Of Cd127+ Group 1 And Group 3 Innate Lymphoid Cells In The Intestinal Lamina Propria. Immunity 43, 146-160.

Björklund, A.K., Forkel, M., Picelli, S., Konya, V., Theorell, J., Friberg, D., Sandberg, R., and Mjösberg, J. (2016). The heterogeneity of human CD127+ innate lymphoid cells revealed by single-cell RNA sequencing. Nat. Immunol. 17, 451-460.

Böiers, C., Carrelha, J., Lutteropp, M., Luc, S., Green, J.C.A., Azzoni, E., Woll, P.S., Mead, A.J., Hultquist, A., Swiers, G., et al. (2013). Lymphomyeloid contribution of an immune-restricted progenitor emerging prior to definitive hematopoietic stem cells. Cell Stem Cell 13, 535-548.

Boos, M.D., Yokota, Y., Eberl, G., and Kee, B.L. (2007). Mature natural killer cell and lymphoid tissue-inducing cell development requires Id2-mediated suppression of E protein activity. J. Exp. Med. 204, 1119-1130.

Bouskra, D., Brézillon, C., Bérard, M., Werts, C., Varona, R., Boneca, I.G., and Eberl, G. (2008). Lymphoid tissue genesis induced by commensals through NOD1 regulates intestinal homeostasis. Nat. 20084567221 456, 507-510.

De Bruijn, M.F.T.R., Speck, N.A., Peeters, M.C.E., and Dzierzak, E. (2000). Definitive 


\section{References}

hematopoietic stem cells first develop within the major arterial regions of the mouse embryo. EMBO J. 19, 2465-2474.

Buonocore, S., Ahern, P.P., Uhlig, H.H., Ivanov, I.I., Littman, D.R., Maloy, K.J., and Powrie, F. (2010). Innate lymphoid cells drive IL-23 dependent innate intestinal pathology. Nature 464.

Von Burg, N., Chappaz, S., Baerenwaldt, A., Horvath, E., Bose Dasgupta, S., Ashok, D., Pieters, J., Tacchini-Cottier, F., Rolink, A., Acha-Orbea, H., et al. (2014). Activated group 3 innate lymphoid cells promote T-cell-mediated immune responses. Proc. Natl. Acad. Sci. U. S. A. 111, $12835-12840$.

Cao, X., Shores, E.W., Hu-Li, J., Anver, M.R., Kelsail, B.L., Russell, S.M., Drago, J., Noguchi, M., Grinberg, A., Bloom, E.T., et al. (1995). Defective lymphoid development in mice lacking expression of the common cytokine receptor $y$ chain. Immunity 2, 223-238.

Castro, G., Liu, X., Ngo, K., De Leon-Tabaldo, A., Zhao, S., Luna-Roman, R., Yu, J., Cao, T., Kuhn, R., Wilkinson, P., et al. (2017). RORyt and RORa signature genes in human Th17 cells. PLoS One 12.

Cella, M., Fuchs, A., Vermi, W., Facchetti, F., Otero, K., Lennerz, J.K.M., Doherty, J.M., Mills, J.C., and Colonna, M. (2009). A human natural killer cell subset provides an innate source of IL-22 for mucosal immunity. Nature $457,722-725$.

Cella, M., Gamini, R., Sécca, C., Collins, P.L., Zhao, S., Peng, V., Robinette, M.L., Schettini, J., Zaitsev, K., Gordon, W., et al. (2019). Subsets of ILC3-ILC1-like cells generate a diversity spectrum of innate lymphoid cells in human mucosal tissues. Nat. Immunol. 20, 980-991.

Chea, S., Perchet, T., Petit, M., Verrier, T., Guy-Grand, D., Banchi, E.-G., Vosshenrich, C.A.J., Santo, J.P. Di, Cumano, A., and Golub, R. (2016a). Notch signaling in group 3 innate lymphoid cells modulates their plasticity. Sci. Signal. 9, ra45-ra45.

Chea, S., Schmutz, S., Berthault, C., Perchet, T., Petit, M., Burlen-Defranoux, O., Goldrath, A.W., Rodewald, H.R., Cumano, A., and Golub, R. (2016b). Single-Cell Gene Expression Analyses Reveal Heterogeneous Responsiveness of Fetal Innate Lymphoid Progenitors to Notch Signaling. Cell Rep. 14, 1500-1516.

Choi, G.B., Yim, Y.S., Wong, H., Kim, S., Kim, H., Kim, S. V., Hoeffer, C.A., Littman, D.R., and Huh, J.R. (2016). The maternal interleukin-17a pathway in mice promotes autism-like phenotypes in offspring. Science (80-. ). 351, 933-939.

Coles, M., Kioussis, D., and Veiga-Fernandes, H. (2010). Cellular and molecular requirements in lymph node and Peyer's patch development. In Progress in Molecular Biology and Translational Science, (Prog Mol Biol Transl Sci), pp. 177-205.

Constantinides, M.G., McDonald, B.D., Verhoef, P.A., and Bendelac, A. (2014). A committed precursor to innate lymphoid cells. Nature 508, 397-401.

Cossarizza, A., Chang, H.D., Radbruch, A., Akdis, M., Andrä, I., Annunziato, F., Bacher, P., Barnaba, V., Battistini, L., Bauer, W.M., et al. (2017). Guidelines for the use of flow cytometry and cell sorting in immunological studies. Eur. J. Immunol. 47, 1584-1797.

Cupedo, T., Vondenhoff, M.F.R., Heeregrave, E.J., de Weerd, A.E., Jansen, W., Jackson, D.G., Kraal, G., and Mebius, R.E. (2004). Presumptive Lymph Node Organizers are Differentially Represented in Developing Mesenteric and Peripheral Nodes. J. Immunol. 173, 2968-2975.

Cupedo, T., Crellin, N.K., Papazian, N., Rombouts, E.J., Weijer, K., Grogan, J.L., Fibbe, W.E., Cornelissen, J.J., and Spits, H. (2009). Human fetal lymphoid tissue-inducer cells are interleukin 17-producing precursors to RORC+ CD127+ natural killer-like cells. Nat. Immunol. 10, 66-74. 
Eberl, G., and Litman, D.R. (2004). Thymic origin of intestinal a $\mathrm{\beta}$ cells revealed by fate mapping of RORyt+ cells. Science (80-. ). 305, 248-251.

Eberl, G., Marmon, S., Sunshine, M.J., Rennert, P.D., Choi, Y., and Littmann, D.R. (2004). An essential function for the nuclear receptor RORyt in the generation of fetal lymphoid tissue inducer cells. Nat. Immunol. 5, 64-73.

Eberl, G., Di Santo, J.P., and Vivier, E. (2014). The brave new world of innate lymphoid cells. Nat. Immunol. 16, 1-5.

Fallon, P.G., Ballantyne, S.J., Mangan, N.E., Barlow, J.L., Dasvarma, A., Hewett, D.R., McIlgorm, A., Jolin, H.E., and McKenzie, A.N.J. (2006). Identification of an interleukin (IL)-25-dependent cell population that provides IL-4, IL-5, and IL-13 at the onset of helminth expulsion. J. Exp. Med. 203, 1105-1116.

Finke, D., Acha-Orbea, H., Mattis, A., Lipp, M., and Kraehenbuhl, J.. P. (2002). CD4+CD3- cells induce Peyer's patch development: role of alpha4beta1 integrin activation by CXCR5. Immunity $17,363-373$.

Finotto, S., Neurath, M.F., Glickman, J.N., Qin, S., Lehr, H.A., Green, F.H.Y., Ackerman, K., Haley, K., Galle, P.R., Szabo, S.J., et al. (2002). Development of spontaneous airway changes consistent with human asthma in mice lacking T-bet. Science (80-. ). 295, 336-338.

Förster, R., Mattis, A.E., Kremmer, E., Wolf, E., Brem, G., Lipp, M., R, F., AE, M., E, K., E, W., et al. (1996). A putative chemokine receptor, BLR1, directs B cell migration to defined lymphoid organs and specific anatomic compartments of the spleen. Cell 87, 1037-1047.

von Freeden-Jeffry, U., Vieira, P., Lucian, L.A., McNeil, T., Burdach, S.E., and Murray, R. (1995). Lymphopenia in interleukin (IL)-7 gene-deleted mice identifies IL-7 as a nonredundant cytokine. J. Exp. Med. 181, 1519-1526.

Gasteiger, G., Fan, X., Dikiy, S., Lee, S.Y., and Rudensky, A.Y. (2015). Tissue residency of innate lymphoid cells in lymphoid and nonlymphoid organs. Science (80-. ). 350, 981-985.

Geiger, T.L., Abt, M.C., Gasteiger, G., Firth, M.A., O'Connor, M.H., Geary, C.D., O'Sullivan, T.E., van den Brink, M.R., Pamer, E.G., Hanash, A.M., et al. (2014). Nfil3 is crucial for development of innate lymphoid cells and host protection against intestinal pathogens. J. Exp. Med. 211, 17231731.

Geremia, A., Arancibia-Cárcamo, C. V., Fleming, M.P.P., Rust, N., Singh, B., Mortensen, N.J., Travis, S.P.L., and Powrie, F. (2011). IL-23-responsive innate lymphoid cells are increased in inflammatory bowel disease. J. Exp. Med. 208, 1127-1133.

Ghaedi, M., Steer, C.A., Martinez-Gonzalez, I., Halim, T.Y.F., Abraham, N., and Takei, F. (2016). Common-Lymphoid-Progenitor-Independent Pathways of Innate and $T$ Lymphocyte Development. Cell Rep. 15, 471-480.

Gladiator, A., Wangler, N., Trautwein-Weidner, K., and LeibundGut-Landmann, S. (2013). Cutting Edge: IL-17-Secreting Innate Lymphoid Cells Are Essential for Host Defense against Fungal Infection. J. Immunol. 190, 521-525.

Glatzer, T., Killig, M., Meisig, J., Ommert, I., Luetke-Eversloh, M., Babic, M., Paclik, D., Blüthgen, N., Seidl, R., Seifarth, C., et al. (2013). RORyt+ Innate Lymphoid Cells Acquire a Proinflammatory Program upon Engagement of the Activating Receptor NKp44. Immunity 38, 1223-1235.

Gordon-Keylock, S., Sobiesiak, M., Rybtsov, S., Moore, K., and Medvinsky, A. (2013). Mouse extraembryonic arterial vessels harbor precursors capable of maturing into definitive HSCs. Blood $122,2338-2345$. 


\section{References}

Goto, Y., Obata, T., Kunisawa, J., Sato, S., Ivanov, I.I., Lamichhane, A., Takeyama, N., Kamioka, M., Sakamoto, M., Matsuki, T., et al. (2014). Innate lymphoid cells regulate intestinal epithelial cell glycosylation. Science (80-. ). 345.

Grivennikov, S.I., Wang, K., Mucida, D., Stewart, C.A., Schnabl, B., Jauch, D., Taniguchi, K., Yu, G.Y., Österreicher, C.H., Hung, K.E., et al. (2012). Adenoma-linked barrier defects and microbial products drive IL-23/IL-17-mediated tumour growth. Nature 491, 254-258.

Guo, Y., MacIsaac, K.D., Chen, Y., Miller, R.J., Jain, R., Joyce-Shaikh, B., Ferguson, H., Wang, I.M., Cristescu, R., Mudgett, J., et al. (2016). Inhibition of RORYT Skews TCRa Gene Rearrangement and Limits T Cell Repertoire Diversity. Cell Rep. 17, 3206-3218.

Gury-BenAri, M., Thaiss, C.A., Serafini, N., Winter, D.R., Giladi, A., Lara-Astiaso, D., Levy, M., Salame, T.M., Weiner, A., David, E., et al. (2016). The Spectrum and Regulatory Landscape of Intestinal Innate Lymphoid Cells Are Shaped by the Microbiome. Cell 166, 1231-1246.e13.

Haddad, R., Lanjuin, A., Madisen, L., Zeng, H., Murthy, V.N., and Uchida, N. (2013). Olfactory cortical neurons read out a relative time code in the olfactory bulb. Nat. Neurosci. 16, 949-957.

Halim, T.Y.F., MacLaren, A., Romanish, M.T., Gold, M.J., McNagny, K.M., and Takei, F. (2012). Retinoic-Acid-Receptor-Related Orphan Nuclear Receptor Alpha Is Required for Natural Helper Cell Development and Allergic Inflammation. Immunity 37, 463-474.

Hanash, A.M., Dudakov, J.A., Hua, G., O'Connor, M.H., Young, L.F., Singer, N. V., West, M.L., Jenq, R.R., Holland, A.M., Kappel, L.W., et al. (2012). Interleukin-22 Protects Intestinal Stem Cells from Immune-Mediated Tissue Damage and Regulates Sensitivity to Graft versus Host Disease. Immunity 37, 339-350.

Harly, C., Cam, M., Kaye, J., and Bhandoola, A. (2018). Development and differentiation of early innate lymphoid progenitors. J. Exp. Med. 215, 249-262.

Hashi, H., Yoshida, H., Honda, K., Fraser, S., Kubo, H., Awane, M., Takabayashi, A., Nakano, H., Yamaoka, Y., and Nishikawa, S.-I. (2001). Compartmentalization of Peyer's Patch Anlagen Before Lymphocyte Entry. J. Immunol. 166, 3702-3709.

He, Y.-W., Beers, C., Deftos, M.L., Ojala, E.W., Forbush, K.A., and Bevan, M.J. (2000). DownRegulation of the Orphan Nuclear Receptor RORyt Is Essential for T Lymphocyte Maturation. J. Immunol. 164, 5668-5674.

Hepworth, M.R., Monticelli, L.A., Fung, T.C., Ziegler, C.G.K., Grunberg, S., Sinha, R., Mantegazza, A.R., Ma, H.-L., Crawford, A., Angelosanto, J.M., et al. (2013). Innate lymphoid cells regulate CD4+ T-cell responses to intestinal commensal bacteria. Nature 498, 113-117.

Hepworth, M.R., Fung, T.C., Masur, S.H., Kelsen, J.R., McConnell, F.M., Dubrot, J., Withers, D.R., Hugues, S., Farrar, M.A., Reith, W., et al. (2015). Group 3 innate lymphoid cells mediate intestinal selection of commensal bacteria-specific CD4+ T cells. Science (80-. ). 348, 1031-1035.

Hernandez, P., Gronke, K., and Diefenbach, A. (2018). A catch-22: Interleukin-22 and cancer. Eur. J. Immunol. 48, 15-31.

Hernández, P.P., Mahlakoiv, T., Yang, I., Schwierzeck, V., Nguyen, N., Guendel, F., Gronke, K., Ryffel, B., Hoelscher, C., Dumoutier, L., et al. (2015). Interferon- $\lambda$ and interleukin-22 cooperate for the induction of interferon-stimulated genes and control of rotavirus infection. Nat. Immunol. $16,698$.

Hernández, P.P., Strzelecka, P.M., Athanasiadis, E.I., Hall, D., Robalo, A.F., Collins, C.M., Boudinot, P., Levraud, J.P., and Cvejic, A. (2018). Single-cell transcriptional analysis reveals ILClike cells in zebrafish. Sci. Immunol. 3, 5265. 
Hoorweg, K., Peters, C.P., Cornelissen, F., Aparicio-Domingo, P., Papazian, N., Kazemier, G., Mjösberg, J.M., Spits, H., and Cupedo, T. (2012). Functional differences between human NKp44and NKp44+ RORC+ innate lymphoid cells. Front. Immunol. 3, 72.

Ishizuka, I.E., Chea, S., Gudjonson, H., Constantinides, M.G., Dinner, A.R., Bendelac, A., and Golub, R. (2016). Single-cell analysis defines the divergence between the innate lymphoid cell lineage and lymphoid tissue-inducer cell lineage. Nat. Immunol. 17, 269-276.

Jenner, R.G., Townsend, M.J., Jackson, I., Sun, K., Bouwman, R.D., Young, R.A., Glimcher, L.H., and Lord, G.M. (2009). The transcription factors T-bet and GATA-3 control alternative pathways of T-cell differentiation through a shared set of target genes. Proc. Natl. Acad. Sci. 106, 1787617881.

JM, P., CF, M., MA, K., MC, A., D, S., TV, G., SR, B., RF, I., EG, P., PJ, T., et al. (2014). Rapid fucosylation of intestinal epithelium sustains host-commensal symbiosis in sickness. Nature 514 , 638-641.

Kanamori, Y., Ishimaru, K., Nanno, M., Maki, K., Ikuta, K., Nariuchi, H., and Ishikawa, H. (1996). Identification of novel lymphoid tissues in murine intestinal mucosa where clusters of c-kit+ IL7R+ Thy1+ lympho-hemopoietic progenitors develop. J. Exp. Med. 184, 1449-1459.

Kang, H.-S., Chin, R.K., Wang, Y., Yu, P., Wang, J., Newell, K.A., and Fu, Y.-X. (2002). Signaling via LTRR on the lamina propria stromal cells of the gut is required for IgA production. Nat. Immunol. 200236 3, 576-582.

Kim, D., Mebius, R.E., MacMicking, J.D., Jung, S., Cupedo, T., Castellanos, Y., Rho, J., Wong, B.R., Josien, R., Kim, N., et al. (2000). Regulation of Peripheral Lymph Node Genesis by the Tumor Necrosis Factor Family Member TRANCE. J. Exp. Med. 192, 1467-1478.

Kim, H.Y., Lee, H.J., Chang, Y.J., Pichavant, M., Shore, S.A., Fitzgerald, K.A., Iwakura, Y., Israel, E., Bolger, K., Faul, J., et al. (2014). Interleukin-17-producing innate lymphoid cells and the NLRP3 inflammasome facilitate obesity-associated airway hyperreactivity. Nat. Med. 20, 54-61.

Kim, M.Y., McConnell, F.M., Gaspal, F.M.C., White, A., Glanville, S.H., Bekiaris, V., Walker, L.S.K., Caamano, J., Jenkinson, E., Anderson, G., et al. (2007). Function of CD4+CD3- cells in relation to $\mathrm{B}$ - and T-zone stroma in spleen. Blood 109, 1602-1610.

Kirchberger, S., Royston, D.J., Boulard, O., Thornton, E., Franchini, F., Szabady, R.L., Harrison, O., and Powrie, F. (2013). Innate lymphoid cells sustain colon cancer through production of interleukin-22 in a mouse model. J. Exp. Med. 210, 917-931.

Kiss, E.A., Vonarbourg, C., Kopfmann, S., Hobeika, E., Finke, D., Esser, C., Diefenbach, A., EA, K., C, V., S, K., et al. (2011). Natural aryl hydrocarbon receptor ligands control organogenesis of intestinal lymphoid follicles. 334, 1561-1565.

Klein Wolterink, R.G.J., Serafini, N., Van Nimwegen, M., Vosshenrich, C.A.J., De Bruijn, M.J.W., Pereira, D.F., Fernandes, H.V., Hendriks, R.W., and Di Santo, J.P. (2013). Essential, dosedependent role for the transcription factor Gata3 in the development of IL-5+ and IL-13+ type 2 innate lymphoid cells. Proc. Natl. Acad. Sci. U. S. A. 110, 10240-10245.

Klose, C.S.N., Kiss, E.A., Schwierzeck, V., Ebert, K., Hoyler, T., D'Hargues, Y., Göppert, N., Croxford, A.L., Waisman, A., Tanriver, Y., et al. (2013). A T-bet gradient controls the fate and function of CCR6-RORyt + innate lymphoid cells. Nature 494, 261-265.

Klose, C.S.N., Flach, M., Möhle, L., Rogell, L., Hoyler, T., Ebert, K., Fabiunke, C., Pfeifer, D., Sexl, V., Fonseca-Pereira, D., et al. (2014). Differentiation of type 1 ILCs from a common progenitor to all helper-like innate lymphoid cell lineages. Cell 157, 340-356.

Kobayashi, M., Shelley, W.C., Seo, W., Vemula, S., Lin, Y., Liu, Y., Kapur, R., Taniuchi, I., and 


\section{References}

Yoshimoto, M. (2014). Functional B-1 progenitor cells are present in the hematopoietic stem celldeficient embryo and depend on Cbf $\beta$ for their development. Proc. Natl. Acad. Sci. U. S. A. 111, 12151-12156.

Koni, P.A., Sacca, R., Lawton, P., Browning, J.L., Ruddle, N.H., and Flavell, R.A. (1997). Distinct Roles in Lymphoid Organogenesis for Lymphotoxins a and $\beta$ Revealed in Lymphotoxin $\beta$-Deficient Mice. Immunity 6, 491-500.

Konya, V., Czarnewski, P., Forkel, M., Rao, A., Kokkinou, E., Villablanca, E.J., Almer, S., Lindforss, U., Friberg, D., Höög, C., et al. (2018). Vitamin D downregulates the IL-23 receptor pathway in human mucosal group 3 innate lymphoid cells. J. Allergy Clin. Immunol. 141, 279-292.

Kruglov, A.A., Grivennikov, S.I., Kuprash, D. V., Winsauer, C., Prepens, S., Seleznik, G.M., Eberl, G., Littman, D.R., Heikenwalder, M., Tumanov, A. V., et al. (2013). Nonredundant function of soluble Ita3 produced by innate lymphoid cells in intestinal homeostasis. Science (80-. ). 342, $1243-1246$.

Kumaravelu, P., Hook, L., Morrison, A.M., Ure, J., Zhao, S., Zuyev, S., Ansell, J., and Medvinsky, A. (2002). Quantitative developmental anatomy of definitive haematopoietic stem cells/long-term repopulating units (HSC/RUs): role of the aorta-gonad-mesonephros (AGM) region and the yolk sac in colonisation of the mouse embryonic liver. Development 129, 4891-4899.

Lazarevic, V., Chen, X., Shim, J.H., Hwang, E.S., Jang, E., Bolm, A.N., Oukka, M., Kuchroo, V.K., and Glimcher, L.H. (2011). T-bet represses TH 17 differentiation by preventing Runx1-mediated activation of the gene encoding RORyt. Nat. Immunol. 12, 96-104.

Lee, J.-Y., Hall, J.A., Pokrovskii, M., Kroehling, L., Wu, L., and Littman, D.R. (2020). RORa enforces stability of the T-helper-17 cell effector program. BioRxiv 2020.12.15.422921.

Lee, J.S., Cella, M., McDonald, K.G., Garlanda, C., Kennedy, G.D., Nukaya, M., Mantovani, A., Kopan, R., Bradfield, C.A., Newberry, R.D., et al. (2011). AHR drives the development of gut ILC22 cells and postnatal lymphoid tissues via pathways dependent on and independent of Notch. Nat. Immunol. 13, 144-152.

Lee, Y., Chin, R.K., Christiansen, P., Sun, Y., Tumanov, A. V., Wang, J., Chervonsky, A. V., and Fu, Y.X. (2006). Recruitment and Activation of Naive T Cells in the Islets by Lymphotoxin $\beta$ Receptor-Dependent Tertiary Lymphoid Structure. Immunity 25, 499-509.

Lehmann, F.M., von Burg, N., Ivanek, R., Teufel, C., Horvath, E., Peter, A., Turchinovich, G., Staehli, D., Eichlisberger, T., Gomez de Agüero, M., et al. (2020). Microbiota-induced tissue signals regulate ILC3-mediated antigen presentation. Nat. Commun. 11, 1-15.

Lim, A.I., Li, Y., Lopez-Lastra, S., Stadhouders, R., Paul, F., Casrouge, A., Serafini, N., Puel, A., Bustamante, J., Surace, L., et al. (2017). Systemic Human ILC Precursors Provide a Substrate for Tissue ILC Differentiation. Cell 168, 1086-1100.e10.

Lo, B.C., Gold, M.J., Hughes, M.R., Antignano, F., Valdez, Y., Zaph, C., Harder, K.W., and McNagny, K.M. (2016). The orphan nuclear receptor ROR alpha and group 3 innate lymphoid cells drive fibrosis in a mouse model of Crohn's disease. Sci. Immunol. 1, eaaf8864-eaaf8864.

Lo, B.C., Canals Hernaez, D., Scott, R.W., Hughes, M.R., Shin, S.B., Underhill, T.M., Takei, F., McNagny, K.M., Hernaez, D.C., Scott, R.W., et al. (2019). The Transcription Factor RORa Preserves ILC3 Lineage Identity and Function during Chronic Intestinal Infection. J. Immunol. 203, 3209-3215.

Lord, G.M., Rao, R.M., Choe, H., Sullivan, B.M., Lichtman, A.H., Luscinskas, F.W., and Glimcher, L.H. (2005). T-bet is required for optimal proinflammatory CD4+ T-cell trafficking. Blood 106, 3432-3439. 
Lügering, A., Ross, M., Sieker, M., Heidemann, J., Williams, I.R., Domschke, W., and Kucharzik, T. (2010). CCR6 identifies lymphoid tissue inducer cells within cryptopatches. Clin. Exp. Immunol. $160,440$.

Lun, A.T.L., McCarthy, D.J., and Marioni, J.C. (2016). A step-by-step workflow for low-level analysis of single-cell RNA-seq data with Bioconductor. F1000Research 5.

Mackley, E.C., Houston, S., Marriott, C.L., Halford, E.E., Lucas, B., Cerovic, V., Filbey, K.J., Maizels, R.M., Hepworth, M.R., Sonnenberg, G.F., et al. (2015). CCR7-dependent trafficking of RORY+ ILCs creates a unique microenvironment within mucosal draining lymph nodes. Nat. Commun. 6.

Male, V., Nisoli, I., Kostrzewski, T., Allan, D.S.J., Carlyle, J.R., Lord, G.M., Wack, A., and Brady, H.J.M. (2014). The transcription factor E4bp4/Nfil3 controls commitment to the NK lineage and directly regulates Eomes and Id2 expression. J. Exp. Med. 211, 635-642.

Mariathasan, S., Matsumoto, M., Baranyay, F., Nahm, M.H., Kanagawa, O., and Chaplin, D.D. (1995). Absence of lymph nodes in lymphotoxin-a(LTa)-deficient mice is due to abnormal organ development, not defective lymphocyte migration. J. Inflamm. 45, 72-78.

Matsuda, J.L., George, T.C., Hagman, J., and Gapin, L. (2007). Temporal Dissection of T-bet Functions. J. Immunol. 178, 3457-3465.

Mazzurana, L., Czarnewski, P., Jonsson, V., Wigge, L., Ringnér, M., Williams, T.C., Ravindran, A., Björklund, A..K., Säfholm, J., Nilsson, G., et al. (2021). Tissue-specific transcriptional imprinting and heterogeneity in human innate lymphoid cells revealed by full-length single-cell RNAsequencing. Cell Res. 31, 554-568.

McGrath, K.E., Frame, J.M., Fromm, G.J., Koniski, A.D., Kingsley, P.D., Little, J., Bulger, M., and Palis, J. (2011). A transient definitive erythroid lineage with unique regulation of the $\beta$-globin locus in the mammalian embryo. Blood 117, 4600-4608.

McGrath, K.E., Frame, J.M., and Palis, J. (2015a). Early hematopoiesis and macrophage development. Semin. Immunol. 27, 379-387.

McGrath, K.E., Frame, J.M., Fegan, K.H., Bowen, J.R., Conway, S.J., Catherman, S.C., Kingsley, P.D., Koniski, A.D., and Palis, J. (2015b). Distinct Sources of Hematopoietic Progenitors Emerge before HSCs and Provide Functional Blood Cells in the Mammalian Embryo. Cell Rep. 11, 18921904.

McInnes, L., Healy, J., Saul, N., and Großberger, L. (2018). UMAP: Uniform Manifold Approximation and Projection. J. Open Source Softw. 3, 861.

Mebius, R.E. (2003). Organogenesis of lymphoid tissues. Nat. Rev. Immunol. 3, 292-303.

Mebius, R.E., Streeter, P.R., Michie, S., Butcher, E.C., and Weissman, I.L. (1996). A developmental switch in lymphocyte homing receptor and endothelial vascular addressin expression regulates lymphocyte homing and permits CD4+ CD3-cells to colonize lymph nodes. Proc. Natl. Acad. Sci. U. S. A. 93, 11019-11024.

Mebius, R.E., Rennert, P., and Weissman, I.L. (1997). Developing lymph nodes collect CD4+CD3$\mathrm{LT} \beta+$ cells that can differentiate to APC, NK cells, and follicular cells but not T or B cells. Immunity 7, 493-504.

Mebius, R.E., Miyamoto, T., Christensen, J., Domen, J., Cupedo, T., Weissman, I.L., and Akashi, K. (2001). The Fetal Liver Counterpart of Adult Common Lymphoid Progenitors Gives Rise to All Lymphoid Lineages, CD45 + CD4 + CD3 - Cells, As Well As Macrophages . J. Immunol. 166, 6593-6601. 


\section{References}

Mielke, L.A., Groom, J.R., Rankin, L.C., Seillet, C., Masson, F., Putoczki, T., and Belz, G.T. (2013). TCF-1 Controls ILC2 and NKp46 + RORyt + Innate Lymphocyte Differentiation and Protection in Intestinal Inflammation. J. Immunol. 191, 4383-4391.

Montaldo, E., Teixeira-Alves, L.G., Glatzer, T., Durek, P., Stervbo, U., Hamann, W., Babic, M., Paclik, D., Stölzel, K., Gröne, J., et al. (2014). Human RORyt+CD34+ cells are lineage-specified progenitors of group 3 RORyt+ innate lymphoid cells. Immunity 41, 988-1000.

Monticelli, L.A., Sonnenberg, G.F., Abt, M.C., Alenghat, T., Ziegler, C.G.K., Doering, T.A., Angelosanto, J.M., Laidlaw, B.J., Yang, C.Y., Sathaliyawala, T., et al. (2011). Innate lymphoid cells promote lung-tissue homeostasis after infection with influenza virus. Nat. Immunol. 12, 1045-1054.

Moore, M.A.S., and Metcalf, D. (1970). Ontogeny of the Haemopoietic System: Yolk Sac Origin of In Vivo and In Vitro Colony Forming Cells in the Developing Mouse Embryo. Br. J. Haematol. $18,279-296$.

Moro, K., Yamada, T., Tanabe, M., Takeuchi, T., Ikawa, T., Kawamoto, H., Furusawa, J.I., Ohtani, M., Fujii, H., and Koyasu, S. (2010). Innate production of TH 2 cytokines by adipose tissueassociated c-Kit+ Sca-1+ lymphoid cells. Nature 463, 540-544.

Müller, A.M., Medvinsky, A., Strouboulis, J., Grosveld, F., and Dzierzakt, E. (1994). Development of hematopoietic stem cell activity in the mouse embryo. Immunity $1,291-301$.

Nagasawa, M., Heesters, B.A., Kradolfer, C.M.A., Krabbendam, L., Martinez-Gonzalez, I., De Bruijn, M.J.W., Golebski, K., Hendriks, R.W., Stadhouders, R., Spits, H., et al. (2019). KLRG1 and NKp46 discriminate subpopulations of human CD117+CRTH2- ILCs biased toward ILC2 or ILC3. J. Exp. Med. 216, 1762-1776.

Neill, D.R., Wong, S.H., Bellosi, A., Flynn, R.J., Daly, M., Langford, T.K.A., Bucks, C., Kane, C.M., Fallon, P.G., Pannell, R., et al. (2010). Nuocytes represent a new innate effector leukocyte that mediates type-2 immunity. Nature 464, 1367-1370.

Oherle, K., Acker, E., Bonfield, M., Wang, T., Gray, J., Lang, I., Bridges, J., Lewkowich, I., Xu, Y., Ahlfeld, S., et al. (2020). Insulin-like Growth Factor 1 Supports a Pulmonary Niche that Promotes Type 3 Innate Lymphoid Cell Development in Newborn Lungs. Immunity 52, 275294.e9.

Ohmura, K., Kawamoto, H., Fujimoto, S., Ozaki, S., Nakao, K., and Katsura, Y. (1999). Emergence of $\mathrm{T}, \mathrm{B}$, and Myeloid Lineage-Committed as well as Multipotent Hemopoietic Progenitors in the Aorta-Gonad- Mesonephros Region of Day 10 Fetuses of the Mouse. J. Immunol. 163, 4788 LP -4795.

Ohmura, K., Kawamoto, H., Lu, M., Ikawa, T., Ozaki, S., Nakao, K., and Katsura, Y. (2001). Immature Multipotent Hemopoietic Progenitors Lacking Long-Term Bone Marrow-Reconstituting Activity in the Aorta-Gonad-Mesonephros Region of Murine Day 10 Fetuses. J. Immunol. 166, 3290-3296.

Okada, S., Markle, J.G., Deenick, E.K., Mele, F., Averbuch, D., Lagos, M., Alzahrani, M., AlMuhsen, S., Halwani, R., Ma, C.S., et al. (2015). IMMUNODEFICIENCIES. Impairment of immunity to Candida and Mycobacterium in humans with bi-allelic RORC mutations. Science 349.

Oliphant, C.J., Hwang, Y.Y., Walker, J.A., Salimi, M., Wong, S.H., Brewer, J.M., Englezakis, A., Barlow, J.L., Hams, E., Scanlon, S.T., et al. (2014). MHCII-mediated dialog between group 2 innate lymphoid cells and CD4+ T cells potentiates type 2 immunity and promotes parasitic helminth expulsion. Immunity 41, 283-295.

Onder, L., Mörbe, U., Pikor, N., Novkovic, M., Cheng, H.W., Hehlgans, T., Pfeffer, K., Becher, B., Waisman, A., Rülicke, T., et al. (2017). Lymphatic Endothelial Cells Control Initiation of Lymph 
Node Organogenesis. Immunity 47, 80-92.e4.

Orange, J.S., Wang, B., Terhorst, C., and Biron, C.A. (1995). Requirement for natural killer cellproduced interferon $\mathrm{y}$ in defense against murine cytomegalovirus infection and enhancement of this defense pathway by interleukin 12 administration. J. Exp. Med. 182, 1045-1056.

Paclik, D., Stehle, C., Lahmann, A., Hutloff, A., and Romagnani, C. (2015). ICOS regulates the pool of group 2 innate lymphoid cells under homeostatic and inflammatory conditions in mice. Eur. J. Immunol. 45, 2766-2772.

Palis, J., Robertson, S., Kennedy, M., Wall, C., and Keller, G. (1999). Development of erythroid and myeloid progenitors in the yolk sac and embryo proper of the mouse. Development 126 , 5073-5084.

Pantelyushin, S., Haak, S., Ingold, B., Kulig, P., Heppner, F.L., Navarini, A.A., and Becher, B. (2012). Roryt + innate lymphocytes and $ү \delta$ T cells initiate psoriasiform plaque formation in mice. J. Clin. Invest. 122, 2252-2256.

Parker, M.E., Barrera, A., Wheaton, J.D., Zuberbuehler, M.K., Allan, D.S.J., Carlyle, J.R., Reddy, T.E., and Ciofani, M. (2020). C-Maf regulates the plasticity of group 3 innate lymphoid cells by restraining the type 1 program. J. Exp. Med. 217.

Pasparakis, M., Alexopoulou, L., Grell, M., Ppizenviaier, K., Bluethmann, H., and Kollias, G. (1997). Peyer's patch organogenesis is intact yet formation of B lymphocyte follicles is defective in peripheral lymphoid organs of mice deficient for tumor necrosis factor and its 55-kDa receptor. Proc. Natl. Acad. Sci. U. S. A. 94, 6319-6323.

Patel, A., Harker, N., Moreira-Santos, L., Ferreira, M., Alden, K., Timmis, J., Foster, K., Garefalaki, A., Pachnis, P., Andrews, P., et al. (2012). Differential RET signaling pathways drive development of the enteric lymphoid and nervous systems. Sci. Signal. 5.

Van De Pavert, S.A., Olivier, B.J., Goverse, G., Vondenhoff, M.F., Greuter, M., Beke, P., Kusser, K., Höpken, U.E., Lipp, M., Niederreither, K., et al. (2009). Chemokine cxcl13 is essential for lymph node initiation and is induced by retinoic acid and neuronal stimulation. Nat. Immunol. 10, 1193-1199.

Van De Pavert, S.A., Ferreira, M., Domingues, R.G., Ribeiro, H., Molenaar, R., Moreira-Santos, L., Almeida, F.F., Ibiza, S., Barbosa, I., Goverse, G., et al. (2014). Maternal retinoids control type 3 innate lymphoid cells and set the offspring immunity. Nature 508, 123-127.

Pearson, C., Thornton, E.E., McKenzie, B., Schaupp, A.L., Huskens, N., Griseri, T., West, N., Tung, S., Seddon, B.P., Uhlig, H.H., et al. (2016). ILC3 GM-CSF production and mobilisation orchestrate acute intestinal inflammation. Elife 5.

Pham, T.A.N., Clare, S., Goulding, D., Arasteh, J.M., Stares, M.D., Browne, H.P., Keane, J.A., Page, A.J., Kumasaka, N., Kane, L., et al. (2014). Epithelial IL-22RA1-mediated fucosylation promotes intestinal colonization resistance to an opportunistic pathogen. Cell Host Microbe 16, 504-516.

Pokrovskii, M., Hall, J.A., Ochayon, D.E., Yi, R., Chaimowitz, N.S., Seelamneni, H., Carriero, N., Watters, A., Waggoner, S.N., Littman, D.R., et al. (2019). Characterization of Transcriptional Regulatory Networks that Promote and Restrict Identities and Functions of Intestinal Innate Lymphoid Cells. Immunity 51, 185-197.e6.

Powell, N., Walker, A.W., Stolarczyk, E., Canavan, J.B., Gökmen, M.R., Marks, E., Jackson, I., Hashim, A., Curtis, M.A., Jenner, R.G., et al. (2012). The Transcription Factor T-bet Regulates Intestinal Inflammation Mediated by Interleukin-7 Receptor+ Innate Lymphoid Cells. Immunity $37,674-684$. 


\section{References}

Price, A.E., Liang, H.E., Sullivan, B.M., Reinhardt, R.L., Eisley, C.J., Erle, D.J., and Locksley, R.M. (2010). Systemically dispersed innate IL-13-expressing cells in type 2 immunity. Proc. Natl. Acad. Sci. U. S. A. 107, 11489-11494.

Qiu, J., Heller, J.J., Guo, X., Chen, Z.E., Fish, K., Fu, Y.-X., and Zhou, L. (2012). The Aryl Hydrocarbon Receptor Regulates Gut Immunity through Modulation of Innate Lymphoid Cells. Immunity 36, 92-104.

Qiu, J., Guo, X., Chen, Z. ming E., He, L., Sonnenberg, G.F., Artis, D., Fu, Y.X., and Zhou, L. (2013). Group 3 innate lymphoid cells inhibit T-cell-mediated intestinal inflammation through aryl hydrocarbon receptor signaling and regulation of microflora. Immunity 39, 386-399.

Rankin, L.C., Groom, J.R., Chopin, M., Herold, M.J., Walker, J.A., Mielke, L.A., McKenzie, A.N.J., Carotta, S., Nutt, S.L., and Belz, G.T. (2013). The transcription factor T-bet is essential for the development of NKp46 + innate lymphocytes via the Notch pathway. Nat. Immunol. 14, 389395.

Rankin, L.C., Girard-Madoux, M.J.H., Seillet, C., Mielke, L.A., Kerdiles, Y., Fenis, A., Wieduwild, E., Putoczki, T., Mondot, S., Lantz, O., et al. (2016). Complementarity and redundancy of IL-22producing innate lymphoid cells. 17, 179-186.

Renoux, V.M., Zriwil, A., Peitzsch, C., Michaëlsson, J., Friberg, D., Soneji, S., and Sitnicka, E. (2015). Identification of a Human Natural Killer Cell Lineage-Restricted Progenitor in Fetal and Adult Tissues. Immunity 43, 394-407.

Roberts, N.A., White, A.J., Jenkinson, W.E., Turchinovich, G., Nakamura, K., Withers, D.R., McConnell, F.M., Desanti, G.E., Benezech, C., Parnell, S.M., et al. (2012). Rank Signaling Links the Development of Invariant $ү \delta \mathrm{T}$ Cell Progenitors and Aire + Medullary Epithelium. Immunity $36,427-437$.

Robinette, M.L., Fuchs, A., Cortez, V.S., Lee, J.S., Wang, Y., Durum, S.K., Gilfillan, S., and Colonna, M. (2015). Transcriptional programs define molecular characteristics of innate lymphoid cell classes and subsets. Nat. Immunol. 16, 306-317.

Robinette, M.L., Bando, J.K., Song, W., Ulland, T.K., Gilfillan, S., and Colonna, M. (2017). IL-15 sustains IL-7R-independent ILC2 and ILC3 development. Nat. Commun. 8, 1-13.

Roelli, P., bbimber, Flynn, B., santiagorevale, and Gui, G. (2019). Hoohm/CITE-seq-Count: 1.4.2.

Saenz, S.A., Siracusa, M.C., Perrigoue, J.G., Spencer, S.P., Urban, J.F., Tocker, J.E., Budelsky, A.L., Kleinschek, M.A., Kastelein, R.A., Kambayashi, T., et al. (2010). IL25 elicits a multipotent progenitor cell population that promotes T H 2 cytokine responses. Nature 464, 1362-1366.

Sanos, S.L., Bui, V.L., Mortha, A., Oberle, K., Heners, C., Johner, C., and Diefenbach, A. (2009). RORyt and commensal microflora are required for the differentiation of mucosal interleukin 22producing NKp46+ cells. Nat. Immunol. 10, 83-91.

Satoh-Takayama, N., Vosshenrich, C.A.J., Lesjean-Pottier, S., Sawa, S., Lochner, M., Rattis, F., Mention, J.J., Thiam, K., Cerf-Bensussan, N., Mandelboim, O., et al. (2008). Microbial flora drives interleukin 22 production in intestinal NKp46+ cells that provide innate mucosal immune defense. Immunity 29, 958-970.

Sawa, S., Cherrier, M., Lochner, M., Satoh-Takayama, N., Fehling, H.J., Langa, F., Di Santo, J.P., and Eberl, G. (2010). Lineage relationship analysis of RORyt+ innate lymphoid cells. Science (80. ). 330,665-669.

Sawa, S., Lochner, M., Satoh-Takayama, N., Dulauroy, S., Bérard, M., Kleinschek, M., Cua, D., Di Santo, J.P., Eberl, G., S, S., et al. (2011). RORyt+ innate lymphoid cells regulate intestinal homeostasis by integrating negative signals from the symbiotic microbiota. Nat. Immunol. 12, 
Schlenner, S.M., Madan, V., Busch, K., Tietz, A., Läufle, C., Costa, C., Blum, C., Fehling, H.J., and Rodewald, H.R. (2010). Fate Mapping Reveals Separate Origins of T Cells and Myeloid Lineages in the Thymus. Immunity 32, 426-436.

Schneider, C., Lee, J., Koga, S., Ricardo-Gonzalez, R.R., Nussbaum, J.C., Smith, L.K., Villeda, S.A., Liang, H.E., and Locksley, R.M. (2019). Tissue-Resident Group 2 Innate Lymphoid Cells Differentiate by Layered Ontogeny and In Situ Perinatal Priming. Immunity 50, 1425-1438.e5.

Schroeder, J.-H., Meissl, K., Hromadová, D., Lo, J.W., Neves, J.F., Howard, J.K., Helmby, H., Powell, N., Strobl, B., and Lord, G.M. (2020). T-Bet Controls Cellularity of Intestinal Group 3 Innate Lymphoid Cells. Front. Immunol. 11.

Sciumè, G., Hirahara, K., Takahashi, H., Laurence, A., Villarino, A.A., Singleton, K.L., Spencer, S.P., Wilhelm, C., Poholek, A.C., Vahedi, G., et al. (2012). Distinct requirements for T-bet in gut innate lymphoid cells. J. Exp. Med. 209, 2331-2338.

Scoville, S.D., Mundy-Bosse, B.L., Zhang, M.H., Chen, L., Zhang, X., Keller, K.A., Hughes, T., Chen, L., Cheng, S., Bergin, S.M., et al. (2016). A Progenitor Cell Expressing Transcription Factor RORyt Generates All Human Innate Lymphoid Cell Subsets. Immunity 44, 1140-1150.

Seehus, C.R., Aliahmad, P., De La Torre, B., Iliev, I.D., Spurka, L., Funari, V.A., and Kaye, J. (2015). The development of innate lymphoid cells requires TOX-dependent generation of a common innate lymphoid cell progenitor. Nat. Immunol. 16, 599-608.

Seillet, C., Rankin, L.C., Groom, J.R., Mielke, L.A., Tellier, J., Chopin, M., Huntington, N.D., Belz, G.T., and Carotta, S. (2014a). Nfil3 is required for the development of all innate lymphoid cell subsets. 211.

Seillet, C., Huntington, N.D., Gangatirkar, P., Axelsson, E., Minnich, M., Brady, H.J.M., Busslinger, M., Smyth, M.J., Belz, G.T., and Carotta, S. (2014b). Differential Requirement for Nfil3 during NK Cell Development. J. Immunol. 192, 2667-2676.

Seillet, C., Mielke, L.A., Amann-Zalcenstein, D.B., Su, S., Gao, J., Almeida, F.F., Shi, W., Ritchie, M.E., Naik, S.H., Huntington, N.D., et al. (2016). Deciphering the Innate Lymphoid Cell Transcriptional Program. Cell Rep. 17, 436-447.

Shikhagaie, M.M., Björklund, Å.K., Mjösberg, J., Erjefält, J.S., Cornelissen, A.S., Ros, X.R., Bal, S.M., Koning, J.J., Mebius, R.E., Mori, M., et al. (2017). Neuropilin-1 Is Expressed on Lymphoid Tissue Residing LTi-like Group 3 Innate Lymphoid Cells and Associated with Ectopic Lymphoid Aggregates. Cell Rep. 18, 1761-1773.

Shinkai, Y., Rathbun, OGary, Lam, K.P., Oltz, E.M., Stewart, V., Mendelsohn, M., Charron, J., Datta, M., Young, F., Stall, A.M., et al. (1992). RAG-2-deficient mice lack mature lymphocytes owing to inability to initiate $V(D)$ J rearrangement. Cell $68,855-867$.

Simic, M., Manosalva, I., Spinelli, L., Gentek, R., Shayan, R.R., Siret, C., Girard-Madoux, M., Wang, S., de Fabritus, L., Verschoor, J., et al. (2020). Distinct Waves from the Hemogenic Endothelium Give Rise to Layered Lymphoid Tissue Inducer Cell Ontogeny. Cell Rep. 32, 108004.

Song, C., Lee, J.S., Gilfillan, S., Robinette, M.L., Newberry, R.D., Stappenbeck, T.S., Mack, M., Cella, M., and Colonna, M. (2015). Unique and redundant functions of NKp46+ ILC3s in models of intestinal inflammation. J. Exp. Med. 212, 1869-1882.

Sonnenberg, G.F., and Artis, D. (2015). Innate lymphoid cells in the initiation, regulation and resolution of inflammation. Nat. Med. 21, 1-11.

Sonnenberg, G.F., Monticelli, L.A., Elloso, M.M., Fouser, L.A., and Artis, D. (2011). CD4+ 


\section{References}

Lymphoid Tissue-Inducer Cells Promote Innate Immunity in the Gut. Immunity 34, 122-134.

Sonnenberg, G.F., Monticelli, L.A., Alenghat, T., Fung, T.C., Hutnick, N.A., Kunisawa, J., Shibata, N., Grunberg, S., Sinha, R., Zahm, A.M., et al. (2012). Innate lymphoid cells promote anatomical containment of lymphoid-resident commensal bacteria. Science (80-. ). 336, 1321-1325.

Spencer, S.P., Wilhelm, C., Yang, Q., Hall, J.A., Bouladoux, N., Boyd, A., Nutman, T.B., Urban, J.F., Wang, J., Ramalingam, T.R., et al. (2014). Adaptation of innate lymphoid cells to a micronutrient deficiency promotes type 2 barrier immunity. Science (80-. ). 343, 432-437.

Spits, H., Bernink, J.H., and Lanier, L. (2016). NK cells and type 1 innate lymphoid cells: partners in host defense. Nat. Immunol. 17, 758-764.

Srinivas, S., Watanabe, T., Lin, C.S., William, C.M., Tanabe, Y., Jessell, T.M., and Costantini, F. (2001). Cre reporter strains produced by targeted insertion of EYFP and ECFP into the ROSA26 locus. BMC Dev. Biol. 1, 1-8.

Street, K., Risso, D., Fletcher, R.B., Das, D., Ngai, J., Yosef, N., Purdom, E., and Dudoit, S. (2018). Slingshot: Cell lineage and pseudotime inference for single-cell transcriptomics. BMC Genomics 19.

Stuart, T., Butler, A., Hoffman, P., Hafemeister, C., Papalexi, E., Mauck, W.M., Hao, Y., Stoeckius, M., Smibert, P., and Satija, R. (2019). Comprehensive Integration of Single-Cell Data. Cell 177, 1888-1902.e21.

Sun, Z., Unutmaz, D., Zou, Y.R., Sunshine, M.J., Pierani, A., Brenner-Morton, S., Mebius, R.E., and Littman, D.R. (2000). Requirement for RORY in thymocyte survival and lymphoid organ development. Science (80-. ). 288, 2369-2373.

Sundrud, M.S., and Rao, A. (2008). Regulation of T Helper 17 Differentiation by Orphan Nuclear Receptors: It's Not Just RORyt Anymore. Immunity 28, 5-7.

Teunissen, M.B.M., Munneke, J.M., Bernink, J.H., Spuls, P.I., Res, P.C.M., Te Velde, A., Cheuk, S., Brouwer, M.W.D., Menting, S.P., Eidsmo, L., et al. (2014). Composition of innate lymphoid cell subsets in the human skin: Enrichment of NCR + ILC3 in lesional skin and blood of psoriasis patients. J. Invest. Dermatol. 134, 2351-2360.

Tizian, C., Lahmann, A., Hölsken, O., Cosovanu, C., Kofoed-Branzk, M., Heinrich, F., Mashreghi, M.F., Kruglov, A., Diefenbach, A., and Neumann, C. (2020). c-Maf restrains t-bet-driven programming of CCR6-negative group 3 innate lymphoid cells. Elife 9.

Tober, J., Koniski, A., McGrath, K.E., Vemishetti, R., Emerson, R., De Mesy-Bentley, K.K.L., Waugh, R., and Palis, J. (2007). The megakaryocyte lineage originates from hemangioblast precursors and is an integral component both of primitive and of definitive hematopoiesis. Blood $109,1433-1441$.

De Togni, P., Goellner, J., Ruddle, N.H., Streeter, P.R., Fick, A., Mariathasan, S., Smith, S.C., Carlson, R., Shornick, L.P., Strauss-Schoenberger, J., et al. (2014). Abnormal development of peripheral lymphoid organs in mice deficient in lymphotoxin. J. Immunol. 192, 2007-2014.

Townsend, M.J., Weinmann, A.S., Matsuda, J.L., Salomon, R., Farnham, P.J., Biron, C.A., Gapin, L., and Glimcher, L.H. (2004). T-bet regulates the terminal maturation and homeostasis of NK and Va14i NKT cells. Immunity 20,477-494.

Tsuji, M., Suzuki, K., Kitamura, H., Maruya, M., Kinoshita, K., Ivanov, I.I., Itoh, K., Littman, D.R., and Fagarasan, S. (2008). Requirement for Lymphoid Tissue-Inducer Cells in Isolated Follicle Formation and T Cell-Independent Immunoglobulin A Generation in the Gut. Immunity 29, 261271. 
Veiga-Fernandes, H., Coles, M.C., Foster, K.E., Patel, A., Williams, A., Natarajan, D., Barlow, A., Pachnis, V., and Kioussis, D. (2007). Tyrosine kinase receptor RET is a key regulator of Peyer's patch organogenesis. Nature 446, 547-551.

Verrier, T., Satoh-Takayama, N., Serafini, N., Marie, S., Di Santo, J.P., and Vosshenrich, C.A.J. (2016). Phenotypic and Functional Plasticity of Murine Intestinal NKp46 + Group 3 Innate Lymphoid Cells. J. Immunol. 196, 4731-4738.

Viant, C., LC, R., MJ, G.-M., C, S., W, S., MJ, S., L, B., T, W., ND, H., E, V., et al. (2016). Transforming growth factor- $\beta$ and Notch ligands act as opposing environmental cues in regulating the plasticity of type 3 innate lymphoid cells. 9 .

Villanova, F., Flutter, B., Tosi, I., Grys, K., Sreeneebus, H., Perera, G.K., Chapman, A., Smith, C.H., Di Meglio, P., and Nestle, F.O. (2014). Characterization of innate lymphoid cells in human skin and blood demonstrates increase of NKp44+ ILC3 in psoriasis. J. Invest. Dermatol. 134, 984-991.

Vonarbourg, C., and Diefenbach, A. (2012). Multifaceted roles of interleukin-7 signaling for the development and function of innate lymphoid cells. Semin. Immunol. 24, 165-174.

Vonarbourg, C., Mortha, A., Bui, V.L., Hernandez, P.P., Kiss, E.A., Hoyler, T., Flach, M., Bengsch, B., Thimme, R., Hölscher, C., et al. (2010). Regulated expression of nuclear receptor RORyt confers distinct functional fates to NK cell receptor-expressing RORyt(+) innate lymphocytes. Immunity 33, 736-751.

Vosshenrich, C.A.J., García-Ojeda, M.E., Samson-Villéger, S.I., Pasqualetto, V., Enault, L., Goff, O.R. Le, Corcuff, E., Guy-Grand, D., Rocha, B., Cumano, A., et al. (2006). A thymic pathway of mouse natural killer cell development characterized by expression of GATA-3 and CD127. Nat. Immunol. 7, 1217-1224.

Walker, J.A., Clark, P.A., Crisp, A., Barlow, J.L., Szeto, A., Ferreira, A.C.F., Rana, B.M.J., Jolin, H.E., Rodriguez-Rodriguez, N., Sivasubramaniam, M., et al. (2019). Polychromic Reporter Mice Reveal Unappreciated Innate Lymphoid Cell Progenitor Heterogeneity and Elusive ILC3 Progenitors in Bone Marrow. Immunity 51, 104-118.e7.

Wolf, F.A., Hamey, F.K., Plass, M., Solana, J., Dahlin, J.S., Göttgens, B., Rajewsky, N., Simon, L., and Theis, F.J. (2019). PAGA: graph abstraction reconciles clustering with trajectory inference through a topology preserving map of single cells. Genome Biol. 20, 1-9.

Wong, S.H., Walker, J.A., Jolin, H.E., Drynan, L.F., Hams, E., Camelo, A., Barlow, J.L., Neill, D.R., Panova, V., Koch, U., et al. (2012). Transcription factor RORa is critical for nuocyte development. Nat. Immunol. 13, 229-236.

Xiong, H., Keith, J.W., Samilo, D.W., Carter, R.A., Leiner, I.M., and Pamer, E.G. (2016). Innate Lymphocyte/Ly6Chi Monocyte Crosstalk Promotes Klebsiella Pneumoniae Clearance. Cell 165, 679-689.

Xu, W., Domingues, R.G., Fonseca-Pereira, D., Ferreira, M., Ribeiro, H., Lopez-Lastra, S., Motomura, Y., Moreira-Santos, L., Bihl, F., Braud, V., et al. (2015). NFIL3 Orchestrates the emergence of common helper innate lymphoid cell precursors. Cell Rep. 10, 2043-2054.

Xu, W., Cherrier, D.E., Chea, S., Vosshenrich, C., Serafini, N., Petit, M., Liu, P., Golub, R., and Di Santo, J.P. (2019). An Id2RFP-Reporter Mouse Redefines Innate Lymphoid Cell Precursor Potentials. Immunity 50, 1054-1068.e3.

Yagi, R., Zhong, C., Northrup, D.L., Yu, F., Bouladoux, N., Spencer, S., Hu, G., Barron, L., Sharma, S., Nakayama, T., et al. (2014). The transcription factor GATA3 is critical for the development of all IL-7Ra-expressing innate lymphoid cells. Immunity 40, 378-388. 


\section{References}

Yamano, T., Dobeš, J., Vobořil, M., Steinert, M., Brabec, T., Ziętara, N., Dobešová, M., Ohnmacht, C., Laan, M., Peterson, P., et al. (2019). Aire-expressing ILC3-like cells in the lymph node display potent APC features. J. Exp. Med. 216, 1027-1037.

Yang, Q., Monticelli, L.A., Saenz, S.A., Chi, A.W.S., Sonnenberg, G.F., Tang, J., De Obaldia, M.E., Bailis, W., Bryson, J.L., Toscano, K., et al. (2013). T Cell Factor 1 Is Required for Group 2 Innate Lymphoid Cell Generation. Immunity 38, 694-704.

Yang, Q., Li, F., Harly, C., Xing, S., Ye, L., Xia, X., Wang, H., Wang, X., Yu, S., Zhou, X., et al. (2015). TCF-1 upregulation identifies early innate lymphoid progenitors in the bone marrow. Nat. Immunol. 16, 1044-1050.

Yang, X.O., Pappu, B.P., Nurieva, R., Akimzhanov, A., Kang, H.S., Chung, Y., Ma, L., Shah, B., Panopoulos, A.D., Schluns, K.S., et al. (2008a). T Helper 17 Lineage Differentiation Is Programmed by Orphan Nuclear Receptors RORa and RORY. Immunity 28, 29-39.

Yang, X.O., Pappu, B.P., Nurieva, R., Akimzhanov, A., Kang, H.S., Chung, Y., Ma, L., Shah, B., Panopoulos, A.D., Schluns, K.S., et al. (2008b). T Helper 17 Lineage Differentiation Is Programmed by Orphan Nuclear Receptors RORa and RORY. Immunity 28, 29-39.

Yokota, Y., Mansouri, A., Mori, S., Sugawara, S., Adachi, S., Nishikawa, S.I., Gruss, P., Y, Y., A, M., S, M., et al. (1999). Development of peripheral lymphoid organs and natural killer cells depends on the helix-loop-helix inhibitor Id2. Nature 397, 702-706.

Yoshida, T., Yao-Ming Ng, S., Zuniga-Pflucker, J.C., and Georgopoulos, K. (2006). Early hematopoietic lineage restrictions directed by Ikaros. Nat. Immunol. 7, 382-391.

Yoshimoto, M., Montecino-Rodriguez, E., Ferkowicz, M.J., Porayette, P., Shelley, W.C., Conway, S.J., Dorshkind, K., and Yoder, M.C. (2011). Embryonic day 9 yolk sac and intra-embryonic hemogenic endothelium independently generate a B-1 and marginal zone progenitor lacking B2 potential. Proc. Natl. Acad. Sci. U. S. A. 108, 1468-1473.

Yoshimoto, M., Porayette, P., Glosson, N.L., Conway, S.J., Carlesso, N., Cardoso, A.A., Kaplan, M.H., and Yoder, M.C. (2012). Autonomous murine T-cell progenitor production in the extraembryonic yolk sac before HSC emergence. Blood 119, 5706-5714.

Yu, Y., Tsang, J.C.H., Wang, C., Clare, S., Wang, J., Chen, X., Brandt, C., Kane, L., Campos, L.S., Lu, L., et al. (2016). Single-cell RNA-seq identifies a PD-1hi ILC progenitor and defines its development pathway. Nature 539, 102-106.

Yudanin, N.A., Schmitz, F., Flamar, A.L., Thome, J.J.C., Tait Wojno, E., Moeller, J.B., Schirmer, M., Latorre, I.J., Xavier, R.J., Farber, D.L., et al. (2019). Spatial and Temporal Mapping of Human Innate Lymphoid Cells Reveals Elements of Tissue Specificity. Immunity 50, 505-519.e4.

Zeis, P., Lian, M., Fan, X., Herman, J.S., Hernandez, D.C., Gentek, R., Elias, S., Symowski, C., Knöpper, K., Peltokangas, N., et al. (2020). In Situ Maturation and Tissue Adaptation of Type 2 Innate Lymphoid Cell Progenitors. Immunity 53, 775-792.e9.

Zheng, G.X.Y., Terry, J.M., Belgrader, P., Ryvkin, P., Bent, Z.W., Wilson, R., Ziraldo, S.B., Wheeler, T.D., McDermott, G.P., Zhu, J., et al. (2017). Massively parallel digital transcriptional profiling of single cells. Nat. Commun. 8 .

Zhong, C., Cui, K., Wilhelm, C., Hu, G., Mao, K., Belkaid, Y., Zhao, K., and Zhu, J. (2016). Group 3 innate lymphoid cells continuously require the transcription factor GATA-3 after commitment. Nat. Immunol. 17, 169-178.

Zhu, J., Jankovic, D., Oler, A.J., Wei, G., Sharma, S., Hu, G., Guo, L., Yagi, R., Yamane, H., Punkosdy, G., et al. (2012). The Transcription Factor T-bet Is Induced by Multiple Pathways and Prevents an Endogenous Th2 Cell Program during Th1 Cell Responses. Immunity 37, 660-673. 
Zook, E.C., Ramirez, K., Guo, X., van der Voort, G., Sigvardsson, M., Svensson, E.C., Fu, Y.X., and Kee, B.L. (2016). The ETS1 transcription factor is required for the development and cytokineinduced expansion of ILC2. J. Exp. Med. 213, 687-696. 


\section{Abbreviations}

Ahr. Aryl Hydrocarbon Receptor

AMP. Anti-Microbial Peptides

BAC. Bacterial Artificial Chromosome

BM. Bone Marrow

CD. Crohn's Disease

CLP. Common Lymphoid Progenitor

CP. Cryptopatches

DAMP. Damage-Associated Molecular Pattern

DKO. Double Knockout (ROR $y$ t and T-bet, $\left.\operatorname{Rorc}(g t)^{G F P / G F P} \times \operatorname{Tb} \times 21^{-/-}\right)$

DN. "double-negative"

DZ. Dark Zone

FL. Fetal Liver

GFP. Green Fluorescent Protein

GC. Germinal Center

HSC. Hematopoietic Stem Cell

IBD. Inflammatory Bowel Disease

IFN. Interferon

ILC. Innate Lymphoid Cell

ILCP. Innate Lymphoid Cell Progenitor

IL. Interleukin

ILF. Isolated Lymphoid Follicle

LN. Lymph Node

LT. Lymphotoxin

LTi. Lymphoid Tissue inducer

LTin. Lymphoid Tissue initiator

LTo. Lymphoid Tissue Organizer 
LZ. Light Zone

M. Microfold (M cells)

MHCII. Major Histocompatibility Complex class II

mLN. Mesenteric Lymph Node

NCR. Natural Cytotoxicity Receptor

NK. Natural Killer

PAMP. Pathogen-Associated Molecular Pattern

PP. Peyer's Patches

RA. Retinoic Acid

Reporter. ROR $\gamma$ t reporter (Rorc(gt $\left.)^{G F P / w t}\right)$

RKO. ROR $\gamma$ t Knockout (Rorc(gt $)^{\text {GFP/GFP) }}$

RNA. Ribonucleic Acid

ROR. Retinoic acid receptor-related Orphan Receptor

scRNA-seq. Single-Cell RNA Sequencing

SCS. Subcapsular Sinus

SI. Small Intestine

TF. Transcription Factor

Th. T helper

Tfh. T follicular helper

UC. Ulcerative Colitis 


\section{List of figures and tables}

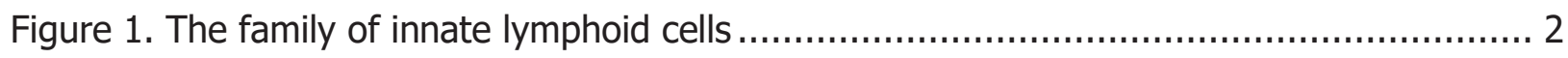

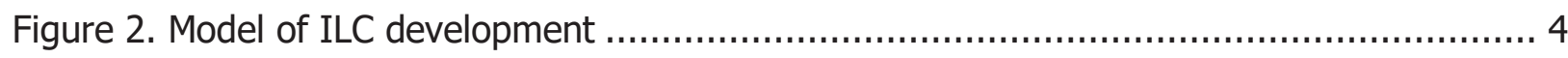

Figure 3. Antagonizing factors regulate ILC3 subsets during ontogeny $\ldots \ldots \ldots \ldots \ldots \ldots \ldots \ldots \ldots \ldots$

Figure 4. Environmental signals and the interplay of and transcription factors ROR $\gamma \mathrm{t}$ and T-bet

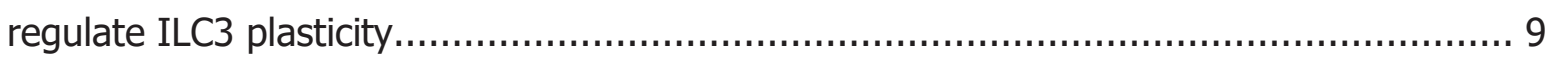

Figure 5. Model for development of secondary lymphoid organs..................................11

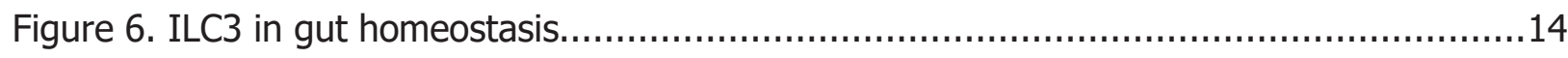

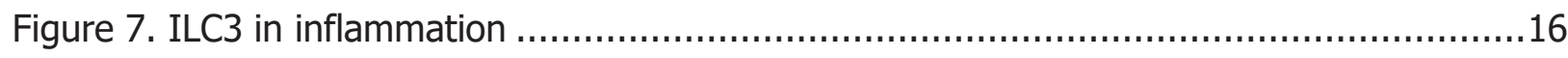

Figure 8. Schematic overview of adoptive transfer experimental setup.........................25

Figure 9. Identification of embryonic ILCs by flow cytometry .................................... 34

Figure 10. Single-cell RNA sequencing of E18.5 intestinal cells identifies distinct clusters........35

- $\quad$ single cell library was prepared with help of Timo Rückert who also provided the R code analysis

Figure 11. Single-cell RNA sequencing of fetal intestinal cells reveals a spectrum of mature ILC

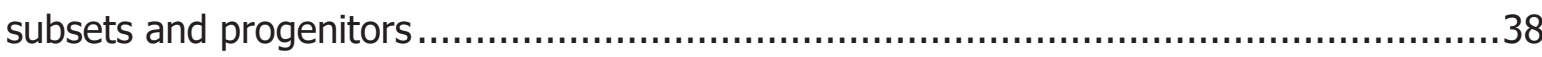

Figure 12. Single-cell RNA sequencing defines CLP and ILCP progenitors within the embryonic intestine

Figure 13. Gene expression patterns of specified embryonic ILC3 subsets defined by scRNAseq.

Figure 14. Transcriptomic profiling on single-cell level discloses heterogeneity within the embryonic ILC3 supercluster

Figure 15. A subset of ROR $\gamma \mathrm{t}^{+} \mathrm{T}^{-}$-bet ${ }^{+} \mathrm{CD} 4^{+}$ILC3 emerges during embryonic development .......43

Figure 16. Kinetics of ILC3 subsets during ontogeny reveal distinct waves of differentiation ...44

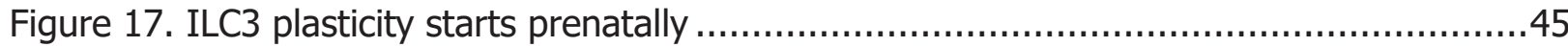

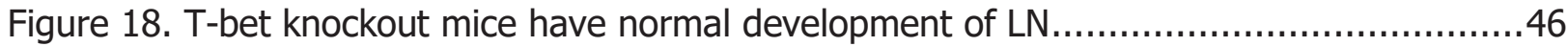

Figure 19. T-bet deficiency rescues LN formation in RORyt-deficient mice ........................46

- The initial observation on the LN phenotype of DKO mice was discovered by Dr. Jakob Zimmermann

Figure 20. LN from ROR $\gamma \mathrm{t} / \mathrm{T}$-bet-DKO mice have normal architecture and are functional .47

- Staining of immunofluorescence samples and acquisition of images by Dr. Carolin Ulbricht.

Figure 21. Re-appearance of $L N$ is independent of RAG 48

Figure 22. Single-cell RNA sequencing of fetal ILC populations reveal accumulation of ILCP in ROR $\gamma \mathrm{t} / \mathrm{T}$-bet-DKO mice 
Figure 23. Gene expressions patterns in ILCP show enhanced LTi expression patterns in DKO mice.

Figure 24. ILCP from DKO mice are enriched in cells with LTi signatures..........................53

Figure 25. Equal Ki67 expression within ILCP of reporter, RKO and DKO mouse strains..........53

Figure 26. In vitro OP9 differentiation cultures....................................................5

Figure 27. PLZF ${ }^{\text {hi }}$ CXCR6 ${ }^{+}$ILCP persist in LN and intestine of adult DKO mice.. .....................55

Figure 28. ROR $\gamma \mathrm{t} / \mathrm{T}$-bet-deficient ILCP produce IL-22 and promote intestinal barrier functions in

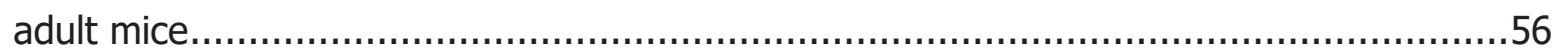

Figure 29. GFP ${ }^{+}$ILCP are generated in in vivo adoptive transfer experimental setup.............57

Figure 30. Schematic overview of Rorc locus and Rorc deletions in mouse models .................58

Figure 31. Thymic $\mathrm{CD}^{+} \mathrm{CD}^{+}$compartments are not restored in $\mathrm{ROR} \gamma \mathrm{t} / \mathrm{T}$-bet double-deficient

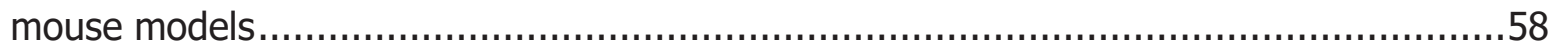

Figure 32. LN formation and accumulation of PLZF+ ILCP is independent of DNA- and ligandbinding domains of ROR $\gamma \mathrm{t}$ in the absence of T-bet............................................... 59

Figure 33. ROR $\alpha$ promotes LN development and PLZF+ ILCP accumulation in DKO mice.........60

Figure 34. Schematical overview on transcription factor networks regulation during ILC differentiation .68

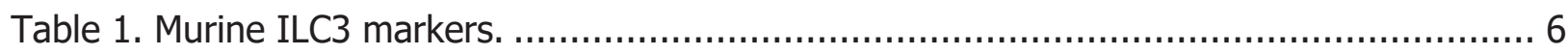

Table 2. Development of LTi cells, $\mathrm{mLN}$ and PP in mutant mice................................... 12

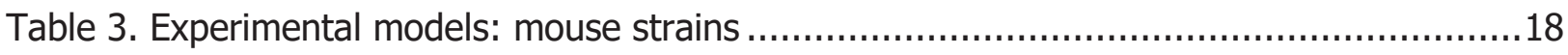

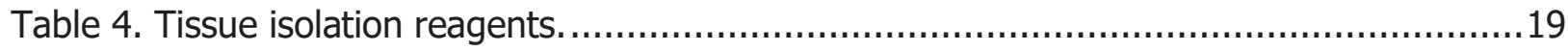

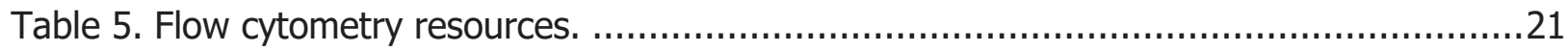

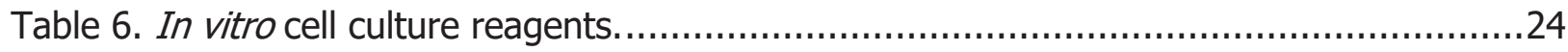

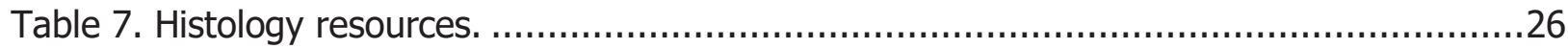

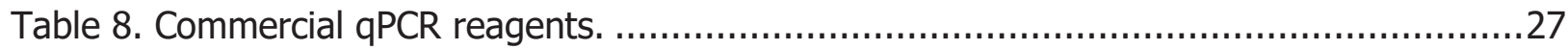

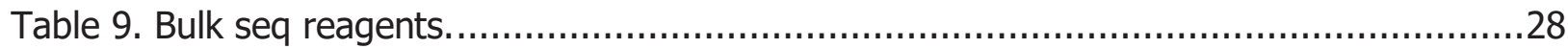

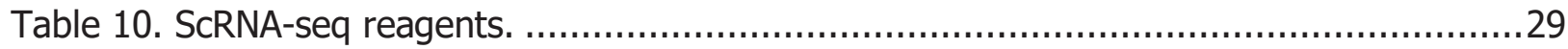

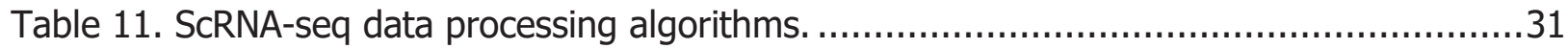

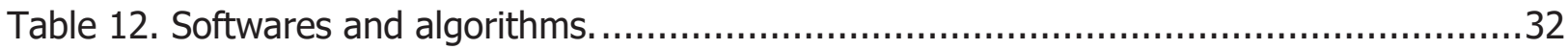




\section{Eidesstattliche Erklärung}

Hiermit erkläre ich an Eides statt, die vorliegende Dissertation selbstständig gemäß guter wissenschaftlicher Praxis angefertigt und keine anderen als die angegeben Hilfsmittel verwendet zu haben.

Weiterhin erkläre ich hiermit, dass die Dissertation oder Teile davon nicht bereits bei einer anderen wissenschaftlichen Einrichtung eingereicht, angenommen oder abgelehnt wurden und auch kein anderwärtiger Doktorgrad erworben wurde.

Berlin, den 30.07.2021

Ergebnisse der vorliegenden Arbeit wurden veröffentlicht:

Stehle, C., Rückert, T., Fiancette, R., Gajdasik, D.W., Willis, C., Ulbricht, C., Durek, P., Mashreghi, M.F., Finke, D., Hauser, A.E., et al. (2021). T-bet and RORa control lymph node formation by regulating embryonic innate lymphoid cell differentiation. Nat. Immunol. 20212210 22, 12311244.

In Kooperation erzeugte Daten sind im Abbildungverzeichnis dieser Arbeit vermerkt. 